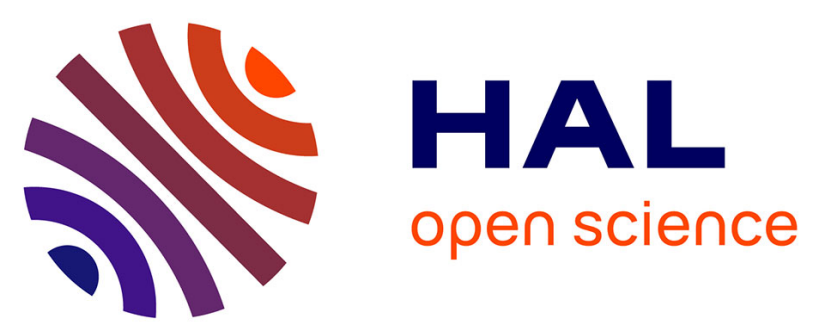

\title{
EMPIRICAL COMPARISON OF METHODS FOR THE HIERARCHICAL PROPAGATION OF HYBRID UNCERTAINTY IN RISK ASSESSMENT, IN PRESENCE OF DEPENDENCES
}

Nicola Pedroni, Enrico Zio

\section{To cite this version:}

Nicola Pedroni, Enrico Zio. EMPIRICAL COMPARISON OF METHODS FOR THE HIERARCHICAL PROPAGATION OF HYBRID UNCERTAINTY IN RISK ASSESSMENT, IN PRESENCE OF DEPENDENCES. International Journal of Uncertainty, Fuzziness and Knowledge-Based Systems, 2012, 20 (4), pp.509-557. 10.1142/S0218488512500250 . hal-00839645

\section{HAL Id: hal-00839645}

https://hal-centralesupelec.archives-ouvertes.fr/hal-00839645

Submitted on 2 Jul 2013

HAL is a multi-disciplinary open access archive for the deposit and dissemination of scientific research documents, whether they are published or not. The documents may come from teaching and research institutions in France or abroad, or from public or private research centers.
L'archive ouverte pluridisciplinaire HAL, est destinée au dépôt et à la diffusion de documents scientifiques de niveau recherche, publiés ou non, émanant des établissements d'enseignement et de recherche français ou étrangers, des laboratoires publics ou privés. 


\title{
EMPIRICAL COMPARISON OF METHODS FOR THE HIERARCHICAL PROPAGATION OF HYBRID UNCERTAINTY IN RISK ASSESSMENT, IN PRESENCE OF DEPENDENCES
}

\author{
NICOLA PEDRONI \\ Energy Department, Politecnico di Milano \\ Via Ponzio, 34/3, Milano, 20133, Italy \\ ENRICO ZIO \\ Chair of system science and the energetic challenge-Electricitè de France, Ecole Centrale Paris and Supelec \\ Grande Voie des Vignes, 92295, Chatenay Malabry-Cedex, France
}

\begin{abstract}
Risk analysis models describing aleatory (i.e., random) events contain parameters (e.g., probabilities, failure rates, ...) that are epistemically-uncertain, i.e., known with poor precision. Whereas aleatory uncertainty is always described by probability distributions, epistemic uncertainty may be represented in different ways (e.g., probabilistic or possibilistic), depending on the information and data available.

The work presented in this paper addresses the issue of accounting for (in)dependence relationships between epistemically-uncertain parameters. When a probabilistic representation of epistemic uncertainty is considered, uncertainty propagation is carried out by a two-dimensional (or double) Monte Carlo (MC) simulation approach; instead, when possibility distributions are used, two approaches are undertaken: the hybrid MC and Fuzzy Interval Analysis (FIA) method and the MCbased Dempster-Shafer (DS) approach employing Independent Random Sets (IRSs). The objectives are: i) studying the effects of (in)dependence between the epistemically-uncertain parameters of the aleatory probability distributions (when a probabilistic/possibilistic representation of epistemic uncertainty is adopted) and ii) studying the effect of the probabilistic/possibilistic representation of epistemic uncertainty (when the state of dependence between the epistemic parameters is defined). The Dependency Bound Convolution (DBC) approach is then undertaken within a hierarchical setting of hybrid (probabilistic and possibilistic) uncertainty propagation, in order to account for all kinds of (possibly unknown) dependences between the random variables.

The analyses are carried out with reference to two toy examples, built in such a way to allow performing a fair quantitative comparison between the methods, and evaluating their rationale and appropriateness in relation to risk analysis.

Keywords: aleatory and epistemic uncertainties, dependences, two-dimensional Monte Carlo method, possibility distributions, fuzzy interval analysis, dependency bound convolution.
\end{abstract}

*Second address: Energy Department, Politecnico di Milano, Via Ponzio, 34/3, 20133 Milano, Italy 


\section{Introduction}

In risk analysis, uncertainty is typically distinguished into two types: randomness due to inherent variability in the system behavior and imprecision due to lack of knowledge and information on the system. The former type of uncertainty is often referred to as objective, aleatory, stochastic whereas the latter is often referred to as subjective, epistemic, state of knowledge. ${ }^{1-4}$

We are interested in the framework of two hierarchical levels of uncertainty, referred to as "level-2" setting:" the models of the aleatory events (e.g., the failure of a mechanical component, the variation of its geometrical dimensions and material properties, ...) contain parameters (e.g., probabilities, failure rates,...) that are epistemically-uncertain, i.e., known with poor precision. ${ }^{\text {a }}$

In current risk analysis, both aleatory and epistemic uncertainties are treated within a probabilistic framework. ${ }^{1,6-9}$ However, in some situations, the lack of complete knowledge, information and data impairs the probabilistic representation of epistemic uncertainty. A number of alternative representation frameworks have been proposed to handle such cases, ${ }^{10-14}$ e.g., fuzzy set theory, ${ }^{15}$ Dempster-Shafer theory of evidence, ${ }^{16-21}$ possibility theory ${ }^{22-26}$ and interval analysis. ${ }^{27-31}$

In this paper, we use probability distributions to describe aleatory uncertainty and we consider both probability and possibility distributions to describe the epistemic uncertainty in the parameters of the (aleatory) probability distributions. ${ }^{23-26}$ When both aleatory and epistemic uncertainties are represented by probability distributions, their propagation is carried out by a two-dimensional (or double) Monte Carlo (MC) simulation approach. ${ }^{5,32,33}$ Instead, when a hybrid (probabilistic and possibilistic) uncertainty representation is considered, two approaches are here undertaken: (i) the hybrid MC and Fuzzy Interval Analysis (FIA) approach, ${ }^{b}$ where the MC technique ${ }^{34,35}$ is combined with the extension principle of fuzzy set theory, ${ }^{36-45}$ within a "level-2" hierarchical setting, ${ }^{24,46-51}$ (ii) the Monte Carlo (MC)-based Dempster-Shafer (DS) approach employing Independent Random Sets (IRSs), ${ }^{c}$ where the possibility distributions describing the epistemically-uncertain parameters are discretized into focal sets that are randomly and independently sampled by MC. ${ }^{52-62}$

\footnotetext{
" This framework of uncertainty modeling is an extension of the so-called "level-1" setting where random variability (aleatory uncertainty) and lack-of-knowledge (epistemic uncertainty) are not separated into two hierarchical levels. ${ }^{5}$

${ }^{\mathrm{b}}$ In the following, this method will be referred to as "hybrid MC-FIA approach" for brevity.

"In the following, this method will be referred to as "MC-based DS-IRS approach" for brevity.
} 
The above mentioned methods encompass several assumptions about the (in)dependence relationships between (i) the epistemically-uncertain parameters of the aleatory probability distributions and (ii) the aleatory variables. With respect to that, two issues must be considered for the practical application of the methods in risk assessment problems:

(i) in the hybrid MC-FIA approach, total dependence is assumed between the epistemically-uncertain parameters of the aleatory probability distributions, i.e., between the information sources (e.g., the experts or observers) that supply the corresponding possibility distributions, ${ }^{23,24}$ on the contrary, in the MC-based DS-IRS approach, random set independence between the epistemic parameters is implied $;^{52-55}$

(ii) the standard MC method (used to propagate the aleatory uncertainties in the three methods mentioned above) presupposes independence between the random variables, $^{63}$ which may lead to overly optimistic results in risk assessment problems. ${ }^{64-66}$ In addition, although some dependences between the random variables may be accounted for by a MC approach (e.g., through copulas ${ }^{67}$ ), not all kinds of possible dependences can be modeled within a MC sampling framework. ${ }^{17}$

The present paper addresses the first issue (i) above by comparing the double MC, hybrid MC-FIA and MC-based DS-IRS approaches with the following objectives:

- the study of the effect of dependence between the epistemically-uncertain parameters of the aleatory probability distributions when a probabilistic/non-probabilistic representation of epistemic uncertainty is adopted;

- the study of the effect of the probabilistic/non-probabilistic representation of epistemic uncertainty when the state of dependence between the epistemic parameters is defined.

With respect to the second issue (ii) above, this paper aims at removing the assumption of independence between random variables. To this aim, the Dependency Bound Convolution (DBC) method ${ }^{17,64,68,69}$ is combined with the Fuzzy Interval Analysis (FIA) approach within a "level-2" framework of hybrid (probabilistic and possibilistic) 
uncertainty propagation in order to account for all kinds of (possibly unknown) dependences between the random variables. ${ }^{d}$

To the best of the authors' knowledge, this is the first time that the above mentioned issues are systematically analyzed with reference to risk assessment problems where hybrid uncertainty is separated into two hierarchical levels. To keep the analysis simple and retain a clear view of each step, the investigations are carried out with respect to two simple examples; in addition, different numerical indicators (e.g., cumulative distributions, exceedance probabilities, percentiles, ...) are considered to perform a fair, quantitative comparison between the methods and evaluate their rationale and appropriateness in relation to risk analysis.

The work benefits from the efforts that have already been done to formalize theoretically the distinct concepts of independence that arise in problems involving both variability and imprecision, ${ }^{70-72}$ within the frameworks of both evidence ${ }^{73-76}$ and possibility theories..$^{77-81}$ The practical implications of different definitions of independence are illustrated with reference to the probabilistic risk assessment of engineering systems by Refs. 17, 54 and 55 only in a "level-1" setting; similar analyses are performed on environmental cases of soil contamination by Refs. 38 and 52, still in a "level-1" setting only.

The remainder of the paper is organized as follows. In Sec. 2, the main steps of the techniques here employed for the joint hierarchical propagation of hybrid uncertainty in a "level-2" framework (i.e., the two-dimensional MC, hybrid MC-FIA, MC-based DS-IRS and the hybrid DBC-FIA methods) are briefly outlined; in Sec. 3, the two academic examples used to perform the comparison between the uncertainty propagation methods are presented; in Sec. 4, the results of the comparisons are reported and commented; Sec. 5 offers a discussion of the results and some conclusions. Finally, some technical details about the two-dimensional MC, hybrid MC-FIA, MC-based DS-IRS and DBC-FIA approaches are given in Appendices A, B, C and D, respectively, for completeness.

\footnotetext{
${ }^{\mathrm{d}}$ In the following, the hybrid probabilistic and possibilistic approach employing the DBC method (instead of standard MC simulation) for the propagation of the aleatory uncertainties in a "level-2" setting will be referred to as "hybrid DBC-FIA approach" for brevity.
} 


\section{Computational methods employed in this study for the joint hierarchical}

\section{propagation of hybrid uncertainty in a "level-2" framework}

In all generality, we consider a model whose output is a function $Z=f\left(Y_{1}, Y_{2}, \ldots, Y_{j}, \ldots, Y_{n}\right)$ of $n$ variables $Y_{j}, j=1,2, \ldots, n$, whose uncertainty is described by probability distributions $\left\{p_{\boldsymbol{\theta}_{j}}^{Y_{j}}\left(y_{j}\right): j=1,2, \ldots, n\right\}$, where $\boldsymbol{\theta}_{j}=\left\{\boldsymbol{\theta}_{j, 1}, \boldsymbol{\theta}_{j, 2}, \ldots, \boldsymbol{\theta}_{j, m_{j}}\right\}$, $j=1,2, \ldots, n$, are the vectors of the corresponding internal parameters. In a "level-2" framework, the parameters $\boldsymbol{\theta}_{j}=\left\{\theta_{j, 1}, \theta_{j, 2}, \ldots, \theta_{j, m_{i}}\right\}, j=1,2, \ldots, n$, of the probability distributions $\left\{p_{\theta}^{Y_{j}}\left(y_{j}\right): j=1,2, \ldots, n\right\}$ are themselves affected by epistemic uncertainty ${ }^{5}$.

Depending on the framework adopted to represent the epistemic uncertainty in the parameters $\boldsymbol{\theta}_{j}, j=1,2, \ldots, n$, different methods for uncertainty propagation are embraced: when probability distributions $\boldsymbol{p}^{\boldsymbol{\theta}_{j}}\left(\boldsymbol{\theta}_{j}\right)=\left\{p^{\theta_{j, 1}}\left(\theta_{j, 1}\right), p^{\theta_{j, 2}}\left(\theta_{j, 2}\right), \ldots, p^{\theta_{j, m_{j}}}\left(\theta_{j, m_{j}}\right)\right\}$, $j=1,2, \ldots, n$, are used, a two-dimensional (or double) Monte Carlo (MC) simulation approach is undertaken (Sec. 2.1); instead, when possibility distributions $\boldsymbol{\pi}^{\boldsymbol{\theta}_{j}}\left(\boldsymbol{\theta}_{j}\right)=\left\{\boldsymbol{\pi}^{\theta_{j, 1}}\left(\theta_{j, 1}\right), \boldsymbol{\pi}^{\theta_{j, 2}}\left(\theta_{j, 2}\right), \ldots, \boldsymbol{\pi}^{\theta_{j, m_{j}}}\left(\theta_{j, m_{j}}\right)\right\}, j=1,2, \ldots, n$, are chosen, two options are here considered: a hybrid MC and Fuzzy Interval Analysis (FIA) approach (Sec. 2.2), and a MC-based Dempster-Shafer (DS) approach employing Independent Random Sets (IRSs) (Sec. 2.3).

\subsection{Two-dimensional Monte Carlo method}

In extreme synthesis, the two main steps of the procedure are: $:^{5,32,33}$

(1) sample $N_{e}$ random realizations $\boldsymbol{\theta}_{j}^{i_{e}}, i_{e}=1,2, \ldots, N_{e}, j=1,2, \ldots, n$, of the parameter vectors $\boldsymbol{\theta}_{j}$ from the probability distributions $\boldsymbol{p}^{\boldsymbol{\theta}_{j}}\left(\boldsymbol{\theta}_{j}\right), j=1,2, \ldots, n$ (outer loop processing epistemic uncertainty by MC simulation);

(2) for each realization $i_{e}=1,2, \ldots, N_{e}$ of epistemic uncertainty, sample $N_{a}$ random realizations $y_{j}^{i_{a}, i_{e}}, i_{a}=1,2, \ldots, N_{a}, j=1,2, \ldots, n$, of the "probabilistic" variables $Y_{j}, j=1,2, \ldots, n$, from the probability distributions $p_{\theta_{j}^{j_{j}}}^{Y_{j}}\left(y_{j}\right), j=1,2, \ldots, n$, conditioned at the values $\boldsymbol{\theta}_{j}^{i_{i}}$ of the epistemically-uncertain parameters $\boldsymbol{\theta}_{j}$ sampled at step (1) above (inner loop processing aleatory uncertainty by MC simulation).

The output of the algorithm is a set of $N_{e}$ empirical Cumulative Distribution

Functions (CDFs) $\left\{F_{i_{e}}^{Z}: i_{e}=1,2, \ldots, N_{e}\right\}$ for the model output $Z$; this set 
$\left\{F_{i_{e}}^{z}: i_{e}=1,2, \ldots, N_{e}\right\}$ has to be post-processed in order to obtain the upper and lower CDFs, $\bar{F}^{z}$ and $\underline{F}^{z}$, respectively, for $Z$. Further details are not given here for brevity sake: the reader is referred to Appendix A.

Notice that the random samplings performed at steps (1) and (2) above may account for possible dependences existing between the epistemically-uncertain parameters and between the aleatory variables, respectively; on the other hand, such dependences can be obviously included in the analysis, only if they can be modeled within a classical MC framework. ${ }^{63}$ Finally, notice that in this work standard MC simulation is used to propagate the aleatory uncertainties in step (2) above, which presupposes independence between the random variables.

\subsection{Hybrid Monte Carlo and Fuzzy Interval Analysis approach}

In the MC-FIA approach, the propagation of the hybrid uncertainty is performed by combining the MC technique ${ }^{34,35}$ with the extension principle of fuzzy set theory ${ }^{36-45}$ within a "level-2" setting by means of the following main steps: $:^{24,46-51}$

(1) select one possibility value $\alpha \in(0,1]$ and the corresponding cuts $A_{\alpha}^{\theta_{j, 1}}, A_{\alpha}^{\theta_{\gamma, 2}}, \ldots, A_{\alpha}^{\theta_{j, \ldots,}}$, $j=1,2, \ldots, \quad n, \quad$ of the possibility distributions $\boldsymbol{\pi}^{\theta_{j}}\left(\boldsymbol{\theta}_{j}\right)=$ $\left\{\pi^{\theta_{j, .}}\left(\theta_{j, 1}\right), \pi^{\theta_{j, 2}}\left(\theta_{j, 2}\right), \ldots, \pi^{\theta_{j, ., j}}\left(\theta_{j, m_{j}}\right)\right\}$ of the epistemically-uncertain parameters $\boldsymbol{\theta}_{j}, j$ $=1,2, \ldots, n$ (outer loop processing epistemic uncertainty by fuzzy interval analysis);

(2) sample $N_{a}$ random intervals $\left[\underline{y}_{j, \alpha}^{i_{a}}, \bar{y}_{j, \alpha}^{i_{j}}\right], i_{a}=1,2, \ldots, N_{a}, j=1,2, \ldots, n$, of the "probabilistic" variables $Y_{j}, j=1,2, \ldots, n$, from the probability distributions $\left\{p_{\boldsymbol{\theta}_{j}}^{Y_{j}}\left(y_{j}\right): j=1,2, \ldots, n\right\}$, letting the epistemically-uncertain parameters $\boldsymbol{\theta}_{j}=\left\{\theta_{j, 1}, \theta_{j, 2}, \ldots, \theta_{j, m}\right\}$ range within the corresponding $\alpha$-cuts $A_{\alpha}^{\theta_{j, 1}}, A_{\alpha}^{\theta_{j, 2}}, \ldots, A_{\alpha}^{\theta_{j, m j}}, j$ $=1,2, \ldots, n$ (found at step (1) above) (inner loop processing aleatory uncertainty by MC simulation);

(3) repeat step (2) above for another possibility value $\alpha \in(0,1]$.

For each interval $A$ of interest contained in the universe of discourse $U_{z}$ of $Z$, the output of the algorithm is represented by a set of plausibility functions 
$\left\{P l_{\alpha}(A): \alpha \in(0,1]\right\}$ and a set of belief functions $\left\{\operatorname{Bel}_{\alpha}(A): \alpha \in(0,1]\right\}$, obtained in correspondence of the different possibility values $\alpha \in(0,1]$ selected at step (1) above; these sets of functions are then synthesized into the plausibility $\operatorname{Pl}(A)$ and belief $\operatorname{Bel}(A)$ of $A$ as $\int_{0}^{1} P l_{\alpha}(A) d \alpha$ and $\int_{0}^{1} B e l_{\alpha}(A) d \alpha$, respectively. ${ }^{24,39}$ Further technical details are not given here for brevity sake: the reader is referred to Appendix B.

It is worth noting that performing an interval analysis on $\alpha$-cuts assumes total dependence between the epistemically-uncertain parameters. Actually, this procedure implies strong dependence between the information sources (e.g., the experts or observers) that supply the input possibility distributions, because the same confidence level $(1-\alpha)$ is chosen to build the $\alpha$-cuts for all the epistemically-uncertain parameters. ${ }^{23}$ In addition, notice that the random sampling performed in step (2) above can account for dependences possibly existing between the aleatory variables; on the other hand, such dependences can be obviously included in the analysis only if they can be modeled within a classical MC framework: ${ }^{63}$ in this work, standard MC simulation is used to propagate the aleatory uncertainties, which presupposes independence between the random variables. Finally, as highlighted in Ref. 23, it is worth noting that this hybrid propagation method clearly assumes independence between the group of probabilistic (i.e., aleatory or random) variables and the group of the possibilistic (i.e., epistemicallyuncertain) parameters of the aleatory probability distributions.

\subsection{Monte Carlo-based Dempster-Shafer approach employing Independent}

\section{Random Sets}

In the MC-based DS-IRS approach, the possibility distributions employed in the hybrid MC-FIA method are encoded into discrete (focal) sets as follows:

(i) determine $q$ (nested) focal sets for the generic possibilistic parameter $\theta$ as the $\alpha$-cuts $A_{\alpha_{t}}=\left[\underline{\theta}_{\alpha_{t}}, \bar{\theta}_{\alpha_{t}}\right], t=1,2, \ldots, q$, with $\alpha_{1}=1>\alpha_{2}>\ldots>\alpha_{q}>\alpha_{q+1}=0 ;$

(ii) build the mass distribution of the focal sets by assigning $m_{\alpha_{t}}=\Delta \alpha_{t}=\alpha_{t}-\alpha_{t+1}$.

In extreme synthesis, the main steps of the procedure are: $:^{52,53}$

(1) sample $N_{e}$ values $\left\{\alpha_{j, i}^{i_{i}}\right\}, i_{e}=1,2, \ldots, N_{e}, i=1,2, \ldots, m_{j}, j=1,2, \ldots, n$, from the discrete distribution $\left\{\left(\alpha_{j, i, t}, m_{\alpha_{j, t}}\right): t=1,2, \ldots, q\right\}$ : these sampled values represent the 


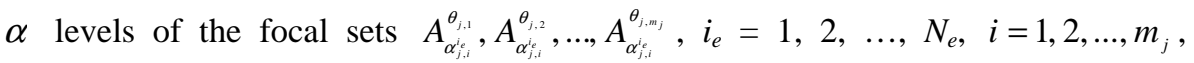
$j=1,2, \ldots, n$, of the discretized possibility distributions $\boldsymbol{\pi}^{\theta_{j}}\left(\boldsymbol{\theta}_{j}\right)$ of the parameters $\boldsymbol{\theta}_{j}, j=1,2, \ldots, n$ (outer loop processing epistemic uncertainty by MC sampling of independent discrete focal sets);

(2) for each realization $i_{e}=1,2, \ldots, N_{e}$ of epistemic uncertainty (step (1) above), sample $N_{a}$ random intervals $\left[\underline{y}_{j}^{i_{a} i_{i}}, \bar{y}_{j}^{i_{j} i_{i}}\right], i_{a}=1,2, \ldots, N_{a}, j=1,2, \ldots, n$, of the "probabilistic" variables $Y_{j}, j=1,2, \ldots, n$, from the probability distributions $\left\{p_{\theta_{j}}^{Y_{j}}\left(y_{j}\right): j=1,2, \ldots, n\right\}$ (see step (2) of the procedure of Sec. 2.2) (inner loop processing aleatory uncertainty by MC simulation).

For each interval $A$ of interest contained in the universe of discourse $U_{Z}$ of $Z$, the output of the algorithm is represented by a set of $N_{e}$ plausibility functions $\left\{P l_{i_{e}}(A): i_{e}=1,2, \ldots, N_{e}\right\}$ and a set of $N_{e}$ belief functions $\left\{\operatorname{Bel}_{i_{e}}(A): i_{e}=1,2, \ldots, N_{e}\right\}$, obtained in correspondence of the $N_{e}$ different combinations of $\alpha$ levels (i.e., of independent random sets) sampled at step 1. above; these sets of functions are then synthesized into the plausibility $P l(A)$ and belief $\operatorname{Bel}(A)$ of $A$ as $P l(A)=\frac{1}{N_{e}} \sum_{i_{e}=1}^{N_{e}} P l_{i_{e}}(A)$ and $\operatorname{Bel}(A)=\frac{1}{N_{e}} \sum_{i_{\iota}=1}^{N_{e}} \operatorname{Bel}_{i_{e}}(A)$, respectively. ${ }^{24,39}$ Further technical details are not given here for brevity sake: the reader is referred to Appendix C.

Notice that, differently from the hybrid MC-FIA approach, at step (1) above a different possibility value (resp., confidence level) $\alpha$ (resp., $1-\alpha$ ) is randomly and independently sampled for each epistemically-uncertain parameter, i.e., random set independence is assumed between the epistemically-uncertain parameters. Again, notice that the random sampling performed in step (2) above can account for dependences possibly existing between the aleatory variables; on the other hand, such dependences can be obviously included in the analysis only if they can be modeled within a classical MC framework: ${ }^{63}$ in this work, standard MC simulation is used to propagate the aleatory uncertainties, which presupposes independence between the random variables. 
Finally, in passing notice that all the methods outlined in Sections 2.1-2.3 employ standard MC simulation to propagate the aleatory uncertainties, which presupposes independence between the random variables $Y_{j}, j=1,2, \ldots, n$. In order to show the possibility of removing this assumption, for illustration purposes the Dependency Bound Convolution (DBC) method ${ }^{17,64,68,69}$ is combined with the Fuzzy Interval Analysis (FIA) approach (Sec. 2.2) in order to account for all kinds of (possibly unknown) dependences between the random variables $Y_{j}, j=1,2, \ldots, n$ : in other words, the DBC method replaces standard MC simulation at step (2) of the procedure in Sec. 2.2. The technical details of the corresponding DBC-FIA algorithm are not given here for brevity: the reader is referred to Appendix D at the end of the paper.

Table 1 summarizes the characteristics of the approaches used in the following to propagate aleatory and epistemic uncertainties in a "level-2" framework. 
Table 1. Characteristics of the approaches considered to propagate aleatory and epistemic uncertainties in a "level-2" framework

\begin{tabular}{ccccc}
\hline Method & $\begin{array}{c}\text { Epistemic uncertainty } \\
\text { representation }\end{array}$ & $\begin{array}{c}\text { Epistemic uncertainty } \\
\text { propagation }\end{array}$ & $\begin{array}{c}\text { State of epistemic } \\
\text { dependence }\end{array}$ & $\begin{array}{c}\text { State of aleatory } \\
\text { dependence }\end{array}$ \\
\hline $\begin{array}{c}\text { Double } \\
\text { MC }\end{array}$ & $\begin{array}{c}\text { Probability } \\
\text { distributions }\end{array}$ & $\begin{array}{c}\text { Random sampling (of } \\
\text { probability distributions) } \\
\text { by MC }\end{array}$ & $\begin{array}{c}\text { Independence/Total } \\
\text { dependence }\end{array}$ & Independence \\
\hline $\begin{array}{c}\text { Hybrid } \\
\text { MC-FIA }\end{array}$ & $\begin{array}{c}\text { Possibility } \\
\text { distributions }\end{array}$ & Fuzzy interval analysis & $\begin{array}{c}\text { Total } \\
\text { dependence }\end{array}$ & Independence \\
\hline $\begin{array}{c}\text { MC-based } \\
\text { DS-IRS }\end{array}$ & $\begin{array}{c}\text { Focal sets } \\
\text { massociated probability } \\
\text { possibility distributions) }\end{array}$ & $\begin{array}{c}\text { Random sampling (of } \\
\text { discrete focal sets) by MC }\end{array}$ & $\begin{array}{c}\text { Random set } \\
\text { independence }\end{array}$ & Independence \\
\hline $\begin{array}{c}\text { Hybrid } \\
\text { DBC-FIA }\end{array}$ & $\begin{array}{c}\text { Possibility } \\
\text { distributions }\end{array}$ & Fuzzy interval analysis & $\begin{array}{c}\text { Total } \\
\text { dependence }\end{array}$ & $\begin{array}{c}\text { Unknown } \\
\text { dependence }\end{array}$ \\
\hline
\end{tabular}

\section{Case studies}

In this Section, the two simple examples adopted as benchmarks to compare the methods of Sec. 2 are presented. In particular, in Sec. 3.1, the model functions used are described together with the representation of the aleatory and epistemic components of uncertainty in the model input variables; in Sec. 3.2, the experimental comparisons carried out throughout the paper are outlined; finally, in Sec. 3.3, the numerical indicators used to perform a quantitative comparison between the uncertainty propagation techniques are provided.

\subsection{Model functions}

Two different model functions are considered:

$$
\begin{aligned}
& Z_{1}=f\left(Y_{1}, Y_{2}, Y_{3}\right)=Y_{1} \cdot Y_{2} \cdot Y_{3} \\
& Z_{2}=f\left(Y_{1}, Y_{2}, Y_{3}\right)=Y_{1} \cdot Y_{2} / Y_{3} .
\end{aligned}
$$

The uncertain input variables $Y_{1}, Y_{2}$ and $Y_{3}$ are described by probability distributions $p_{\boldsymbol{\theta}_{1}}^{Y_{1}}\left(y_{1}\right), p_{\boldsymbol{\theta}_{2}}^{Y_{2}}\left(y_{2}\right)$ and $p_{\boldsymbol{\theta}_{3}}^{Y_{3}}\left(y_{3}\right)$ whose parameter vectors $\boldsymbol{\theta}_{1}, \boldsymbol{\theta}_{2}$ and $\boldsymbol{\theta}_{3}$ are themselves affected by epistemic uncertainty. In particular, $Y_{1}$ is represented by a lognormal distribution $L N\left(\mu_{1}, \sigma_{1}\right)=L N\left(\boldsymbol{\theta}_{1}\right)=L N\left(\theta_{1,1}, \theta_{1,2}\right)$, where $\mu_{1}=\theta_{1,1}$ is described by a triangular possibility distribution $\pi^{\mu_{1}}\left(\mu_{1}\right)=\pi^{\theta_{1,1}}\left(\theta_{1,1}\right)$ with core $c_{1}=8$ and support $\left[a_{1}\right.$, $\left.b_{1}\right]=[7,10]$ and $\sigma_{1}=\theta_{1,2}=1.5 ; Y_{2}$ is represented by a lognormal distribution $L N\left(\mu_{2}, \sigma_{2}\right)=$ $\operatorname{LN}\left(\boldsymbol{\theta}_{2}\right)=\operatorname{LN}\left(\theta_{2,1}, \theta_{2,2}\right)$, where $\mu_{2}=\theta_{2,1}=9$ and $\sigma_{2}=\theta_{2,2}$ is described by a triangular 
possibility distribution $\pi^{\sigma_{2}}\left(\sigma_{2}\right)=\pi^{\theta_{2,2}}\left(\theta_{2,2}\right)$ with core $c_{2}=1.7$ and support $\left[a_{2}, b_{2}\right]=[1$, 2]; finally, $Y_{3}$ is represented by a triangular probability distribution $\operatorname{TR}(l, m, u)=\operatorname{TR}\left(\boldsymbol{\theta}_{3}\right)=$ $\operatorname{TR}\left(\theta_{3,1}, \theta_{3,2}, \theta_{3,3}\right)$, where $l=\theta_{3,1}=1, u=\theta_{3,3}=10$ and $m=\theta_{3,2}$ is described by a triangular possibility distribution $\pi^{m}(m)=\pi^{\theta_{3,2}}\left(\theta_{3,2}\right)$ with core $c_{3}=4$ and support $\left[a_{3}, b_{3}\right]=[2,9]$.

Notice that the simplicity of functions (1) and (2) allows to retain a clear view of (i) the steps involved in the comparison of the uncertainty propagation methods of Sec. 2 and (ii) the "practical" interpretations of the results in relation to possible risk assessment applications.

\subsection{Experimental comparisons}

The following approaches are considered and compared in the task of hierarchically propagating aleatory and epistemic uncertainties in a "level-2" framework:

(i) the two-dimensional (double) MC approach of Sec. 2.1 and Appendix A:

(a) assuming total dependence between the epistemically uncertain parameters of the aleatory probability distributions. This choice has been made to perform a fair comparison with the hybrid Monte Carlo (MC) and Fuzzy Interval Analysis (FIA) approach, which implicitly assumes by construction total dependence between the epistemically uncertain parameters (see Sec. 2.2). ${ }^{\mathrm{e}}$

(b) assuming independence between the epistemically uncertain parameters of the aleatory probability distributions. This choice has been made to perform a fair comparison with the Monte Carlo (MC)-based Dempster-Shafer (DS) approach employing Independent Random Sets (IRSs), which assumes independence between the epistemically uncertain parameters (see Sec. 2.3);

(ii) the hybrid MC-FIA approach of Sec. 2.2 and Appendix B;

(iii) the MC-based DS-IRS approach of Sec. 2.3 and Appendix C.

(iv) the hybrid DBC-FIA approach of Appendix D.

It is worth remembering that, as highlighted in Sections 2.1-2.3 and Table 1, in methods (i)-(iii) above standard MC simulation is used to propagate the aleatory uncertainties, which presupposes independence between the random variables.

${ }^{\mathrm{e}}$ It is important to note that the condition of total epistemic (or state-of-knowledge) dependence between
parameters of risk models is far from unlikely. For example, consider the case of a system containing a number
of physically distinct, but similar/nominally identical components whose failure rates are estimated by means of 
It is worth noting that the representation of epistemic uncertainty here used in the MC-based DS-IRS approach entirely relies on the possibilistic representation described in Sec. 3.1 and employed by the hybrid MC-FIA approach. However, in order to tailor this possibilistic representation to the DS framework, the possibility distributions of Sec. 3.1 are discretized into focal sets (or intervals), each of which is assigned a probability mass as explained in Sec. $2.3:^{23}$ in particular, in this paper $q=20$ (nested) sets are determined for the generic possibilistic parameter $\theta$ as the $\alpha$-cuts $A_{\alpha_{t}}=\left[\underline{\theta}_{\alpha_{t}}, \bar{\theta}_{\alpha_{t}}\right]$, $t=1,2, \ldots, q=20$, with $\alpha_{1}=1>\alpha_{2}>\ldots>\alpha_{q=20}>\alpha_{q+1=21}=0$ and the corresponding mass distribution $m_{\alpha_{t}}$ is built by assigning $m_{\alpha_{t}}=\Delta \alpha_{t}=\alpha_{t}-\alpha_{t+1}=0.05$ : these particular values are chosen for the sake of comparison with the hybrid MC-FIA approach described in detail in Appendix B.

In addition, notice that the probability distributions here used in the two-dimensional MC approach for the epistemically-uncertain parameters are obtained by transforming the possibility distributions of Sec. 3.1 according to the principle of insufficient reason. ${ }^{82}$ The sampling procedure for transforming the possibility distribution $\pi^{\theta}(\theta)$ of the generic parameter $\theta$ into a probability distribution according to this principle is: ${ }^{82,83}$

(i) sample a random realization $\alpha^{*}$ for $\alpha$ in $[0,1)$ and consider the $\alpha$-cut level $A_{\alpha^{*}}=\left[\underline{\theta}_{\alpha^{*}}, \bar{\theta}_{\alpha^{*}}\right]=\left\{\theta: \pi^{\theta}(\theta) \geq \alpha^{*}\right\}$

(ii) sample a random realization $\theta^{*}$ for $\theta$ from a uniform probability distribution on $A_{\alpha^{*}}$.

It is worth noting that other techniques of transformation of possibility distributions into probability density functions have been suggested in the literature, but the corresponding details are not given here for brevity sake: the interested reader is referred to Refs. 41, 82, 84 and 85 for some proposed techniques, e.g., the converse transformation. $^{84}$

Two classes of analyses are performed (Sec. 4):

(1) methods (i)-(iii) above are compared with the following objectives (Sec. 4.1):

the same data set: in such situation, the distributions describing the uncertainty associated to the failure rates have to be considered totally dependent. ${ }^{1,7}$ 
- studying the effects of the state of dependence between the epistemicallyuncertain parameters of the aleatory probability distributions when a probabilistic/non-probabilistic representation of epistemic uncertainty is given: to this aim, approaches that represent in the same way the epistemic uncertainty (i.e., in terms of probability or possibility distributions) but assume different relationships (i.e., dependence or independence) between the epistemically uncertain parameters are compared in the following Sec. 4.1.1 (in particular, comparisons are performed between approaches (i.a) and (i.b) above and between approaches (ii) and (iii) above);

- studying the effects of the probabilistic/non-probabilistic representations of the epistemically-uncertain parameters of the aleatory probability distributions when the state of dependence between the epistemically-uncertain parameters is given: to this aim, approaches assuming the same dependence relationship between the epistemically-uncertain parameters but employing different representations of the epistemic uncertainty are compared in the following Sec. 4.1.2 (in particular, comparisons are performed between approaches (i.a) and (ii) above and between approaches (i.b) and (iii) above).

Again, notice that, as highlighted in Sections 2.1-2.3 and Table 1, in methods (i)-(iii) above standard MC simulation is used to propagate the aleatory uncertainties, which presupposes independence between the random variables.

(2) methods (ii) and (iv) above, i.e., the hybrid MC- and DBC-FIA approaches (assuming independence and unknown dependence between the aleatory variables, respectively) are compared on the academic examples of the previous Sec. 3.1 in order to show the possibility of (Sec. 4.2):

- removing the assumption of independence between the aleatory variables (which is implicit in the adoption of standard MC sampling for the propagation of aleatory uncertainty);

- accounting for all kinds of (possibly unknown) dependences between the aleatory variables (i.e., also those that cannot be modeled even within arbitrarily complex MC sampling frameworks, e.g., copulas).

Table 2 summarizes the analyses carried out in the present paper together with the corresponding objectives. 
Table 2. Comparisons performed in Sec. 4, and their relative objectives

\begin{tabular}{|c|c|c|c|}
\hline \multicolumn{4}{|c|}{ Comparison between the uncertainty propagation methods (Sec. 4.1) } \\
\hline \multicolumn{4}{|c|}{ Sec. 4.1.1 } \\
\hline & & \multicolumn{2}{|c|}{ Representation of epistemic uncertainty } \\
\hline & & Probabilistic & Non-probabilistic \\
\hline \multirow{5}{*}{$\begin{array}{l}\text { State of epistemic } \\
\text { dependence }\end{array}$} & Independence & Double MC (i.b.) & MC-based DS-IRS (iii.) \\
\hline & & vs & vs \\
\hline & $\begin{array}{c}\text { Total } \\
\text { dependence }\end{array}$ & Double MC (i.a.) & Hybrid MC-FIA (ii.) \\
\hline & & $\downarrow$ & $\downarrow$ \\
\hline & Objective & \multicolumn{2}{|c|}{$\begin{array}{l}\text { Study the effects of the state of dependence between the } \\
\text { epistemically-uncertain parameters of the aleatory probability } \\
\text { distributions when a probabilistic/non-probabilistic } \\
\text { representation of epistemic uncertainty is given }\end{array}$} \\
\hline \multicolumn{4}{|c|}{ Sec. 4.1.2 } \\
\hline & & \multicolumn{2}{|c|}{ State of epistemic dependence } \\
\hline & & Independence & Total dependence \\
\hline \multirow{5}{*}{$\begin{array}{l}\text { Representation of } \\
\text { epistemic uncertainty }\end{array}$} & Probabilistic & Double MC (i.b.) & Double MC (i.a.) \\
\hline & & vs & vs \\
\hline & \begin{tabular}{c|c} 
Non- \\
probabilistic
\end{tabular} & MC-based DS-IRS (iii.) & Hybrid MC-FIA (ii.) \\
\hline & & $\downarrow$ & $\downarrow$ \\
\hline & Objective & \multicolumn{2}{|c|}{$\begin{array}{l}\text { Study the effects of the probabilistic/non-probabilistic } \\
\text { representation of the epistemically-uncertain parameters of the } \\
\text { aleatory probability distributions when the state of dependence } \\
\text { between the epistemically uncertain parameters is given }\end{array}$} \\
\hline \multicolumn{4}{|c|}{ Unknown dependences between aleatory variables by DBC (Sec. 4.2) } \\
\hline & & \multicolumn{2}{|c|}{ State of dependence between the aleatory variables } \\
\hline & & Independence & Unknown dependence \\
\hline \multicolumn{2}{|c|}{ Methods } & Hybrid MC-FIA (ii.) & Hybrid DBC-FIA (iv.) \\
\hline Objectiv & & \multicolumn{2}{|c|}{$\begin{array}{l}\text {-Remove the assumption of independence between the aleatory } \\
\text { variables (implicit in the adoption of standard MC sampling for } \\
\text { the propagation of aleatory uncertainty) } \\
\text { - Account for all kinds of (possibly unknown) dependences } \\
\text { between the aleatory variables (i.e., also those that cannot be } \\
\text { modeled even within arbitrarily complex MC sampling } \\
\text { frameworks) }\end{array}$} \\
\hline
\end{tabular}

\subsection{Quantitative indicators}

The experimental comparisons described in the previous Sec. 3.2 are carried out with reference to three quantities of interest in risk assessment: i) the upper and lower Cumulative Distribution Functions (CDFs) $\bar{F}^{z_{i}}\left(z_{i}\right)$ and $\underline{F}^{z_{i}}\left(z_{i}\right)$ of the model output $Z_{i}, i$ $=1,2$; ii) the 2.5-th and 97.5-th percentiles $F^{Z_{i}, 0.025}\left(z_{i}\right)$ and $F^{Z_{i}, 0.975}\left(z_{i}\right)$ of the $\operatorname{CDF} F^{Z_{i}}$ of $Z_{i}, i=1,2$ (i.e., the two CDFs that envelop $95 \%$ of the CDFs of $Z_{i}$ ); iii) the upper and lower CDFs $\bar{F} \bar{Z}_{i .}^{0.95}\left(z_{i}^{0.95}\right)$ and $\underline{F}^{Z_{i .9}^{0.9}}\left(z_{i}^{0.95}\right)$ of the 95 -th percentile $Z_{i}^{0.95}$ of $Z_{i}, i=1,2$. 
The calculation of CDFs is of great importance in risk assessment since they summarize the uncertainty "contained" in the variables of interest, i.e., the model outputs. In the two-dimensional MC approach (Sec. 2.1), the upper and lower CDFs $\bar{F}^{z_{i}}\left(z_{i}\right)$ and $\underline{F}^{z_{i}}\left(z_{i}\right)$ of $Z_{i}, \quad i=1,2$, are computed as $\bar{F}^{z_{i}}\left(z_{i}\right)=\max _{i_{e}=1,2, \ldots, N_{e}}\left\{F_{i_{e}}^{z_{i}}\left(z_{i}\right)\right\}$ and $\underline{F}^{Z_{i}}\left(z_{i}\right)=\min _{i_{i}=1,2, \ldots, N_{e}}\left\{F_{i_{e}}^{z_{i}}\left(z_{i}\right)\right\}$ (i.e., as the two "extreme" CDFs that envelop all the $N_{e}$ CDFs generated in correspondence of the $N_{e}$ realizations of epistemic uncertainty). Instead, in the hybrid MC-FIA (Sec. 2.2) and MC-based DS-IRS (Sec. 2.3) approaches, $\bar{F}^{z_{i}}\left(z_{i}\right)$ and $\underline{F}^{z_{i}}\left(z_{i}\right)$ of $Z_{i}, i=1,2$, are computed by considering the plausibility and belief of the set $A_{i}=\left(-\infty, z_{i}\right], i=1,2$ : in this respect, $\operatorname{Pl}\left(Z_{i} \in\left(-\infty, z_{i}\right]\right)$ and $\operatorname{Bel}\left(Z_{i} \in\left(-\infty, z_{i}\right]\right)$ can be interpreted as bounding cumulative distributions $\bar{F}^{z_{i}}\left(z_{i}\right)=\operatorname{Pl}\left(Z_{i} \in\left(-\infty, z_{i}\right]\right)$ and $\underline{F}^{Z_{i}}\left(z_{i}\right)=\operatorname{Bel}\left(Z_{i} \in\left(-\infty, z_{i}\right]\right), i=1,2$. As highlighted in Ref. 24, $\operatorname{Pl}\left(Z_{i} \in\left(-\infty, z_{i}\right]\right)$ and $\operatorname{Bel}\left(Z_{i} \in\left(-\infty, z_{i}\right]\right)$ are the most precise bounds for the true $\operatorname{CDF} F^{z_{i}}\left(z_{i}\right)$ of $Z_{i}, i=1,2$.

In order to provide a fair and quantitative comparison between the uncertainty propagation methods, two synthetic numerical indicators are also computed based on the functions $\bar{F}^{z_{i}}\left(z_{i}\right)$ and $\underline{F}^{Z_{i}}\left(z_{i}\right)$ : (a) the (interval for the) probability $P\left[Z_{i}>z_{i}^{*}\right]$ that $Z_{i}$ exceeds a given threshold $z_{i}^{*}, i=1,2$ (in the present paper, $z_{1}^{*}=1000$ and $z_{2}^{*}=35$ ); (b) the (interval for the) 95 -th percentile $Z_{i}^{0.95}$ of $Z_{i}, i=1,2$.

However, it has to be noticed that in the two-dimensional MC method the identification of the upper and lower $\operatorname{CDFs} \bar{F}^{z_{i}}\left(z_{i}\right)$ and $\underline{F}^{z_{i}}\left(z_{i}\right)$ of $Z_{i}, i=1,2$, may not provide a faithful representation of the real probabilistic bounds for $F^{z_{i}}\left(z_{i}\right), i=1,2$ : actually, the computation of these CDFs as $\bar{F}^{z_{i}}\left(z_{i}\right)=\max _{i_{e}=1,2, \ldots, N_{e}}\left\{F_{i_{e}}^{z_{i}}\left(z_{i}\right)\right\}$ and $\underline{F}^{z_{i}}\left(z_{i}\right)=\min _{i=1,2, \ldots, N_{e}}\left\{F_{i_{i}}^{z_{i}}\left(z_{i}\right)\right\}$ may be influenced by the occasional random sampling of "extreme" combinations of the epistemic parameters. For example, referring to model function $Z_{1}=Y_{1} \cdot Y_{2} \cdot Y_{3}(1)$ above, it can be seen that a combination of high values of the random variables $Y_{1}$ and $Y_{2}$ and $Y_{3}$ leads to "extremely high" values of the model output 
$Z_{1}$ : notice that in a "level-2" framework of uncertainty modeling, this combination of high values of the random variables $Y_{1}$ and $Y_{2}$ and $Y_{3}$ is favored on its turn by a combination of high values of the corresponding epistemically-uncertain parameters $\mu_{1}=$ $\theta_{1,1}$ and $\sigma_{2}=\theta_{2,2}$ and $m=\theta_{3,2}$, respectively. By way of example, it is evident that if the epistemically-uncertain mean $\mu_{1}=\theta_{1,1}$ of random variable $Y_{1}$ is high, then the values of the corresponding random variable $Y_{1}$ are "expected" to be relatively high (in other words, relatively high values of random variable $Y_{1}$ are favored). Conversely, an occasional combination of low values of the random variables $Y_{1}$ and $Y_{2}$ and $Y_{3}$ produces "extremely low" values of the model output $Z_{1}$ : again, notice that in a "level-2" framework of uncertainty modeling, this combination of low values of the random variables $Y_{1}$ and $Y_{2}$ and $Y_{3}$ is favored on its turn by a combination of low values of the corresponding epistemically-uncertain parameters $\mu_{1}=\theta_{1,1}$ and $\sigma_{2}=\theta_{2,2}$ and $m=\theta_{3,2}$, respectively. Since the upper and lower CDFs $\bar{F}^{z_{1}}\left(z_{1}\right)$ and $\underline{F}^{z_{1}}\left(z_{1}\right)$ are computed by resorting to "max" and "min" operators (i.e., $\bar{F}^{Z_{1}}\left(z_{1}\right)=\max _{i_{e}=1,2, \ldots, N_{e}}\left\{F_{i_{e}}^{Z_{e}}\left(z_{1}\right)\right\}$ and $\underline{F}^{Z_{1}}\left(z_{1}\right)=\min _{i_{e}=1,2, \ldots, N_{e}}\left\{F_{i_{e}}^{Z_{1}}\left(z_{1}\right)\right\}$ ), it may happen that the separation between these functions (in other words, the "content" of epistemic uncertainty carried by them) is entirely determined by the occasional random sampling of even only one of these "extreme" situations, thus not providing a faithful representation of the real probabilistic bounds for $F^{z_{1}}\left(z_{1}\right)$.

In order to overcome this drawback and provide more robust estimates for the probabilistic bounds of $F^{Z_{i}}\left(z_{i}\right)$, the 2.5-th and 97.5-th percentiles $F^{Z_{i}, 0.025}\left(z_{i}\right)$ and $F^{Z_{i}, 0.975}\left(z_{i}\right)$ of the CDF $F^{Z_{i}}$ are here considered. By definition, $F^{Z_{i}, 0.025}\left(z_{i}\right)$ and $F^{Z_{i}, 0.975}\left(z_{i}\right)$ are the two CDFs that envelop $95 \%$ of the CDFs of $Z_{i}, i=1,2:$ in particular, $F^{Z_{i}, 0.025}\left(z_{i}\right)$ is such that $\left(0.025 \cdot N_{e}\right) \%$ of the $N_{e}$ CDFs "lie below" (i.e., are lower than or equal to) $F^{Z_{i}, 0.025}\left(z_{i}\right)$; instead, $F^{Z_{i}, 0.975}\left(z_{i}\right)$ is such that $\left[(1-0.975) \cdot N_{e}\right] \%=\left(0.025 \cdot N_{e}\right) \%$ of the $N_{e}$ CDFs "lie above" (i.e., are larger than or equal to) $F^{z_{i}, 0.975}\left(z_{i}\right)$. The same "empirical" procedure is employed to identify $F^{Z_{i}, 0.025}\left(z_{i}\right)$ and $F^{Z_{i}, 0.975}\left(z_{i}\right)$ in the MC- 
based DS-IRS approach. Notice that the percentiles $F^{z_{i}, 0.025}\left(z_{i}\right)$ and $F^{z_{i, 0.075}}\left(z_{i}\right)$ thereby identified represent more robust estimates of the "true" probabilistic bounds of $F^{z_{i}}\left(z_{i}\right)$ than the upper and lower CDFs $\bar{F}^{z_{i}}\left(z_{i}\right)=\max _{i_{i}=1,2, \ldots, N_{e}}\left\{F_{i_{i}}^{z_{i}}\left(z_{i}\right)\right\}$ and $\underline{F}^{z_{i}}\left(z_{i}\right)=\min _{i_{i}=1,2, \ldots, N_{e}}\left\{F_{i_{e}}^{z_{i}}\left(z_{i}\right)\right\}$ because their identification is not based on "max-min" operations, but rather on order statistics performed on a (possibly) large number $N_{e}$ of realizations, which is less influenced by single "outliers" (i.e., by "extreme" combinations of epistemic parameters values). Instead, in the hybrid MC-FIA approach such percentile distributions are simply obtained by considering the belief and plausibility functions generated in correspondence of the possibility level $\alpha=1-0.95=0.05$ : in particular, $F^{z_{i, 0}, 0.25}\left(z_{i}\right)=$ $\operatorname{Bel}_{0.05}\left(Z_{i} \in\left(-\infty, z_{i}\right]\right)$ and $F^{z_{i}, 0.975}\left(z_{i}\right)=P l_{0.05}\left(Z_{i} \in\left(-\infty, z_{i}\right]\right), i=1,2$.

For the sake of completeness, as before two synthetic numerical indicators are computed based on the functions $F^{z_{i}, 0.025}\left(z_{i}\right)$ and $F^{z_{i}, 0.975}\left(z_{i}\right)$ : (a) the interval $\left[F^{Z_{i}, 0.975}\left(z_{i}^{*}\right), F^{Z_{i} 0.025}\left(z_{i}^{*}\right)\right]$ for the probability $P\left[Z_{i}>z_{i}^{*}\right]$ that $Z_{i}$ exceeds a given threshold $z_{i}^{*}, i=1,2$; (b) the interval $\left[\left(F^{z_{0} 0.975}\right)^{-1}(0.95),\left(F^{z_{i} 0.025}\right)^{-1}(0.95)\right]$ for the 95 -th quantile $Z_{i}^{0.95}$ of $Z_{i}, i=1,2$.

Finally, the upper and lower CDFs $\bar{F}^{z_{i}^{0.95}}\left(z_{i}^{0.95}\right)$ and $\underline{F}^{Z_{i .9}^{095}}\left(z_{i}^{0.95}\right)$ of the 95-th quantile $Z_{i}^{0.95}$ of $Z_{i}, i=1,2$, are considered. In the double MC approach, a single CDF $F^{Z_{i}^{0.95}}\left(z_{i}^{0.95}\right)$ $=\bar{F}^{Z_{i}^{0.95}}\left(z_{i}^{0.95}\right)=\underline{F}^{z_{i}^{0.5}}\left(z_{i}^{0.95}\right)$ for $Z_{i}^{0.95}$ is "empirically" constructed using the $N_{e}$ values $\left\{\left[F_{i_{e}}^{Z_{i}}\right]^{-1}(0.95): i_{e}=1,2, \ldots, N_{e}\right\}$ generated in correspondence of the $N_{e}$ random realizations of epistemic uncertainty: in particular, $F^{Z_{i}^{0.5}}\left(z_{i}^{0.95}\right)=\frac{1}{N_{e}} \sum_{i_{e}=1}^{N_{s}} I\left\{\left[F_{i_{i}}^{Z_{i}}\right]^{-1}(0.95) \leq z_{i}^{0.95}\right\}$, where $\left.I\left[F_{i_{e}}^{Z_{i}}\right]^{-1}(0.95) \leq z_{i}^{0.95}\right\}$ is 1 , if $\left[F_{i_{e}}^{Z_{i}}\right]^{-1}(0.95) \leq z_{i}^{0.95}$ and 0 , otherwise. Similarly, in the MC-based DS-IRS approach, $\bar{F} \bar{F}^{z_{i}^{0.95}}\left(z_{i}^{0.95}\right)=P l^{Z_{i}^{0.95}}\left(z_{i}^{0.95}\right)$ and $\underline{F}^{Z_{i .95}^{0.05}}\left(z_{i}^{0.95}\right)=$ $B e l^{Z_{i}^{0.5}}\left(z_{i}^{0.95}\right)$ are built using the sets of $N_{e}$ values $\left\{\left[P l_{i_{e}}^{Z_{i}}\right]^{-1}(0.95): i_{e}=1,2, \ldots, N_{e}\right\}$ and $\left\{\left[\mathrm{Bel}_{i_{e}}^{z_{i}}\right]^{-1}(0.95): i_{e}=1,2, \ldots, N_{e}\right\}$, respectively. Instead, in the hybrid MC-FIA approach, 
$\bar{F}^{Z_{i}^{0.95}}\left(z_{i}^{0.95}\right)=P l^{Z_{i}^{0.95}}\left(z_{i}^{0.95}\right)$ and $\underline{F}^{z_{i}^{0.95}}\left(z_{i}^{0.95}\right)=\operatorname{Bel}^{Z_{i}^{0.95}}\left(z_{i}^{0.95}\right)$ are obtained as $\sup _{\left[P l_{\alpha}^{Z_{i}}\right]^{-1}(0.95) \leq z_{i}^{0.95}}\{\alpha\}$

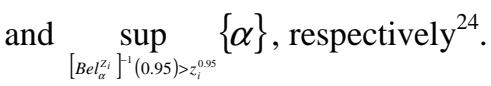

As before, two synthetic numerical indicators are computed based on the functions $\bar{F}^{Z_{i}^{0.55}}\left(z_{i}^{0.95}\right)$ and $\underline{F}^{z_{i}^{0.9}}\left(z_{i}^{0.95}\right):$ (a) the (interval for the) probability $P\left[Z_{i}^{0.95}>z_{i}^{0.95^{*}}\right]$ that $Z_{i}^{0.95}$ exceeds a given threshold $z_{i}^{0.95^{*}}, i=1,2$ (in the present paper, $z_{1}^{0.95^{*}}=1000$ and $z_{2}^{0.95^{*}}=$ 55); (b) the (interval for the) 95 -th quantile $\left[Z_{i}^{0.95}\right]^{0.95}$ of $Z_{i}^{0.95}, i=1,2$.

\section{Applications}

The uncertainty propagation methods described in Sec. 2 are here applied to the examples of Sec. 3: in Sec. 4.1, the efficiency of the methods (i) - (iii) is compared in the task of jointly hierarchically propagating hybrid uncertainty in a "level-2" framework; in Sec. 4.2, the Dependency Bound Convolution (DBC) method and the Fuzzy Interval Analysis (FIA) approach are joined within a "level-2" framework of hybrid (probabilistic and possibilistic) uncertainty propagation in the task of accounting for unknown dependences between the aleatory variables.

\subsection{Comparison of the methods for the joint hierarchical propagation of hybrid uncertainty in a "level-2" framework}

The double Monte Carlo (MC) approach (Sec. 2.1), the hybrid MC and Fuzzy Interval Analysis (FIA) method (Sec. 2.2) and the MC-based Dempster-Shafer approach employing Independent Random Sets (IRSs) (Sec. 2.3) are here compared with the following objectives:

- the study of the effect of dependence between the epistemically-uncertain parameters of the aleatory probability distributions when a probabilistic/non-probabilistic representation of epistemic uncertainty is adopted (Sec. 4.1.1);

- the study of the effect of the probabilistic/non-probabilistic representation of epistemic uncertainty when the state of dependence between the epistemicallyuncertain parameters is defined (Sec. 4.1.2).

It is worth remembering that, as highlighted in Sections 2.1-2.3 and Table 1, in methods (i)-(iii) above (Sec. 3.2) standard MC simulation is used to propagate the aleatory uncertainties, which presupposes independence between the random variables. 


\subsubsection{Dependences between the epistemically-uncertain distribution parameters}

We start by comparing approaches (i.a) and (i.b) above, i.e., double MC assuming total dependence and independence between the uncertain parameters, respectively (Sec. 2.1): the upper and lower Cumulative Distribution Functions (CDFs) $\bar{F}^{z_{i}}\left(z_{i}\right)$ and $\underline{F}^{z_{i}}\left(z_{i}\right), i=$ 1,2 , of the model outputs $Z_{1}=Y_{1} \cdot Y_{2} \cdot Y_{3}(1)$ and $Z_{2}=Y_{1} \cdot Y_{2} / Y_{3}$ (2) obtained by approaches (i.a) and (i.b) are shown in Fig. 1, left and right, respectively.
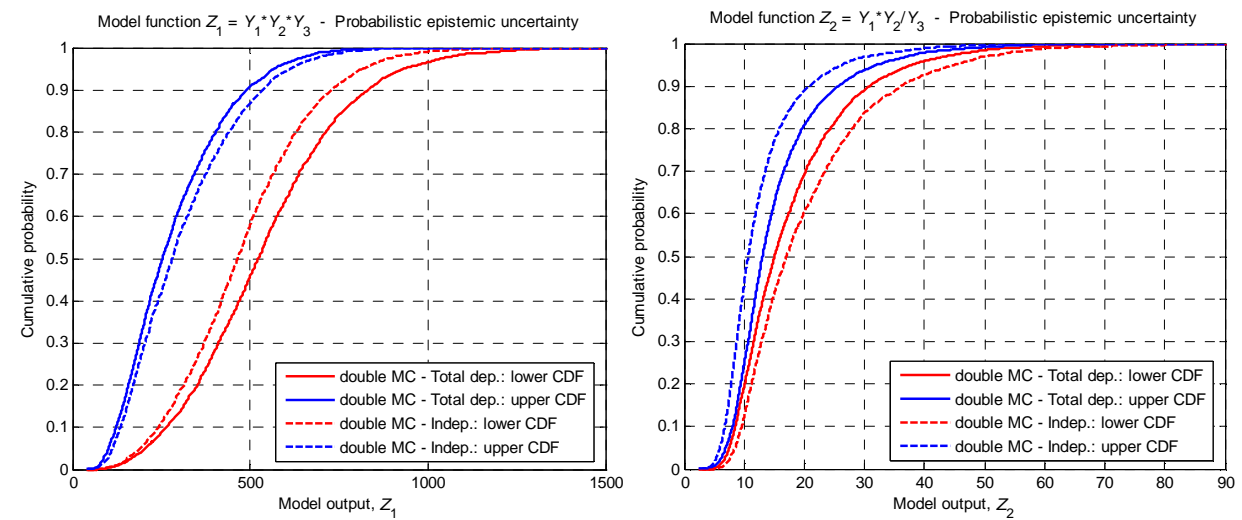

Fig. 1. Comparison of the upper and lower $\operatorname{CDFs} \bar{F}^{Z_{i}}\left(z_{i}\right)$ and $F^{Z_{i}}\left(z_{i}\right), i=1,2$, of the model outputs $Z_{1}=$ $Y_{1} \cdot Y_{2} \cdot Y_{3}$ (left) and $Z_{2}=Y_{1} \cdot Y_{2} / Y_{3}$ (right) obtained by the two-dimensional MC approach, considering total dependence (solid lines) and independence (dashed lines) between the epistemically-uncertain parameters

It can be seen that assuming total dependence between the uncertain parameters leads to a larger gap between the upper and lower CDFs of the model output $Z_{1}=Y_{1} \cdot Y_{2} \cdot Y_{3}$ (1) than assuming independence (Fig. 1, left); instead, the opposite situation occurs for $Z_{2}=$ $Y_{1} \cdot Y_{2} / Y_{3}$ (2) (Fig. 1, right). This can be easily explained by analyzing the input-output functional relationships of the models (1) and (2).

In model function $Z_{2}=Y_{1} \cdot Y_{2} / Y_{3}$ (2) two of the input random variables (i.e., $Y_{1}$ and $Y_{2}$ ) appear at the numerator, whereas the other (i.e., $Y_{3}$ ) appears at the denominator of the expression. In such a case, the highest possible values for the model output $Z_{2}$ are obtained with a combination of high values of both random variables $Y_{1}$ and $Y_{2}$ and low values of random variable $Y_{3}$ : notice that in a "level-2" framework of uncertainty modeling, this particular combination of values of the random variables $Y_{1}, Y_{2}$ and $Y_{3}$ is favored on its turn by a combination of high values of both epistemically-uncertain parameters $\mu_{1}=\theta_{1,1}$ and $\sigma_{2}=\theta_{2,2}$ and low values of epistemically-uncertain parameter $m$ $=\theta_{3,2}$. By way of example, it is evident that if the epistemically-uncertain mean $\mu_{1}=\theta_{1,1}$ of random variable $Y_{1}$ is relatively high, then the values of the corresponding random 
variable $Y_{1}$ are expected to be relatively high (in other words, relatively high values of random variable $Y_{1}$ are favored by high values of the corresponding epistemicallyuncertain mean $\mu_{1}=\theta_{1,1}$ ). Conversely, the lowest possible values for the model output $Z_{2}$ are obtained with a combination of low values of both $Y_{1}$ and $Y_{2}$ and high values of $Y_{3}$ : notice that in a "level-2" framework of uncertainty modeling, this particular combination of values of the random variables $Y_{1}, Y_{2}$ and $Y_{3}$ is favored on its turn by a combination of low values of both epistemically-uncertain parameters $\mu_{1}=\theta_{1,1}$ and $\sigma_{2}=\theta_{2,2}$ and high values of epistemically-uncertain parameter $m=\theta_{3,2}$. These extreme situations (which give rise to the largest separation between the upper and lower CDFs, i.e., to the most "epistemically-uncertain" and, thus, conservative case), can be obtained only in case (i.b) above, i.e., assuming independence between the epistemically-uncertain parameters. Actually, if a pure random sampling is performed among independent epistemicallyuncertain parameters, all possible combinations of values can be in principle generated, since the entire ranges of variability of the epistemically-uncertain parameters can be explored independently: thus, in some random samples of epistemic uncertainty (step (1) of Sec. 2.1), high values of both epistemically-uncertain parameters $\mu_{1}=\theta_{1,1}$ and $\sigma_{2}=\theta_{2,2}$ (which favor on their turn high values of both random variables $Y_{1}$ and $Y_{2}$ ) may be combined by chance with low values of epistemically-uncertain parameter $m=\theta_{3,2}$ (which favor on their turn low values of random variable $Y_{3}$ ); on the contrary, in other random samples of epistemic uncertainty (step (1) of Sec. 2.1), low values of both epistemically-uncertain parameters $\mu_{1}=\theta_{1,1}$ and $\sigma_{2}=\theta_{2,2}$ (which favor on their turn low values of both random variables $Y_{1}$ and $Y_{2}$ ) may be combined by chance with high values of epistemically-uncertain parameter $m=\theta_{3,2}$ (which favor on their turn high values of random variable $Y_{3}$ ). Conversely, such "extreme" situations cannot occur if there is total dependence between the epistemically-uncertain parameters (i.e., case (i.a) above). Actually, in such a case high (resp., low) values of both epistemically-uncertain parameters $\mu_{1}=\theta_{1,1}$ and $\sigma_{2}=\theta_{2,2}$ (which favor on their turn high - resp., low - values of both random variables $Y_{1}$ and $Y_{2}$ ) can only be combined with high (resp., low) values of epistemically-uncertain parameter $m=\theta_{3,2}$ (which favor on their turn high - resp., low values of random variable $Y_{3}$ ), giving rise to values of output $Z_{2}$ which are lower (resp., higher) than the highest (resp., lowest) possible: in other words, the separation between 
the upper and lower CDFs produced in case (i.a) is always smaller than that produced by the "extreme" situations described above (which are possible only in case (i.b)). ${ }^{\mathrm{f}}$

On the contrary, in model function $Z_{1}=Y_{1} \cdot Y_{2} \cdot Y_{3}$ (1) only multiplications (i.e., operations increasing in each place) are present. In such a case, the highest possible values for the model output $Z_{1}$ are obtained with a combination of high values of $Y_{1}$ and $Y_{2}$ and $Y_{3}$ (which are favored on their turn by high values of the corresponding epistemically-uncertain parameters $\mu_{1}=\theta_{1,1}$ and $\sigma_{2}=\theta_{2,2}$ and $\left.m=\theta_{3,2}\right)$; conversely, the lowest possible values for model output $Z_{1}$ are obtained with a combination of low values of $Y_{1}$ and $Y_{2}$ and $Y_{3}$ (which are favored on their turn by low values of the corresponding epistemically-uncertain parameters $\mu_{1}=\theta_{1,1}$ and $\sigma_{2}=\theta_{2,2}$ and $m=\theta_{3,2}$ ). Although these "extreme" situations may be obtained (by chance) also in case (i.b) above (i.e., by assuming independence between the epistemically-uncertain parameters), they can be obtained far more easily (i.e., more probably) in case (i.a) above (i.e., assuming total dependence).

These considerations are supported by the values of the synthetic numerical indicators described in Sec. 3.3. Table 3 reports the intervals for $P\left[Z_{i}>z_{i}^{*}\right]$ and $Z_{i}^{0.95}, i=1,2$, produced by the double MC approaches considering total dependence (case i.a.) and independence (case i.b.) between the epistemically-uncertain parameters. It can be seen that $P\left[Z_{1}>z_{1}^{*}\right]$ and $Z_{1}^{0.95}$ range within $[0.0006,0.0342]$ and $[566.86,932.13]$, respectively, in case (i.a), whereas they range within [0.0004, 0.0092] and [613.58, 816.07], respectively, in case (i.b): thus, for model function (1) the assumption of independence would lead to underestimating the upper bounds of $P\left[Z_{1}>z_{1}^{*}\right]$ and $Z_{1}^{0.95}$ by about $73.1 \%$ and $12.5 \%$, respectively. Instead, $P\left[Z_{2}>z_{2}^{*}\right]$ and $Z_{2}^{0.95}$ range within [0.0626, 0.1108] and [32.12, 38.05], respectively, in case (i.a), whereas they range $[0.0318,0.1640]$ and $[26.36,45.03]$, respectively, in case (i.b): thus, for model function

\footnotetext{
${ }^{\mathrm{f}}$ A straightforward remark is in order. Based on the consideration made above about model $Z_{2}=Y_{1} \cdot Y_{2} / Y_{3}(2)$, the easiest way to sample these "extreme" combinations of parameter values (i.e., to obtain the largest possible separation between the upper and lower CDFs and, thus, the most conservative results) would be by "artificially" imposing total dependence between the epistemically-uncertain parameters of $Y_{1}$ and $Y_{2}$ and opposite dependence between the epistemically-uncertain parameter of $Y_{3}$ and the epistemically-uncertain parameters of both $Y_{1}$ and $Y_{2}$.
} 
(2) it is the assumption of total dependence that leads to underestimate the upper bounds of $P\left[Z_{2}>z_{2}^{*}\right]$ and $Z_{2}^{0.95}$ by about $32.4 \%$ and $15.5 \%$, respectively. ${ }^{\mathrm{g}}$

We now move on to compare methods (ii) and (iii), i.e., the hybrid MC-FIA (Sec. 2.2) and MC-based DS-IRS (Sec. 2.3) approaches. Fig. 2 shows the plausibility and belief functions, $\operatorname{Pl}\left(Z_{i} \in\left(-\infty, z_{i}\right]\right)=\bar{F}^{z_{i}}\left(z_{i}\right)$ and $\operatorname{Bel}\left(Z_{i} \in\left(-\infty, z_{i}\right]\right)=\underline{F}^{Z_{i}}\left(z_{i}\right), i=1,2$, respectively, of the model outputs $Z_{1}=Y_{1} \cdot Y_{2} \cdot Y_{3}$ (1) (left) and $Z_{2}=Y_{1} \cdot Y_{2} / Y_{3}$ (2) (right) produced by the hybrid MC-FIA (solid lines) and MC-based DS-IRS (dashed lines) approaches.
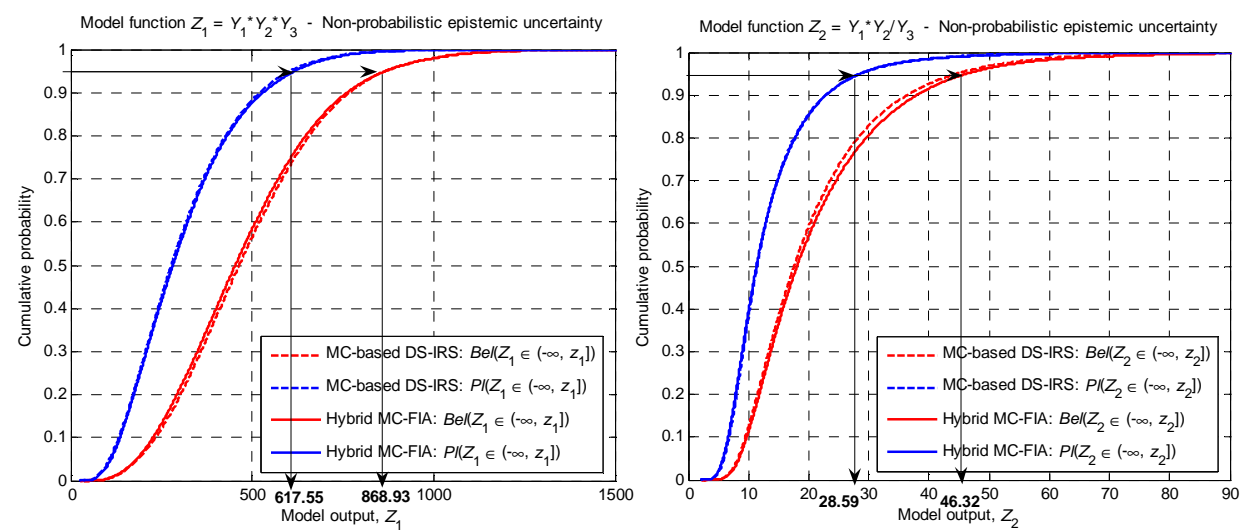

Fig. 2. Comparison of the plausibility and belief functions, $P l\left(Z_{i} \in\left(-\infty, z_{i}\right]\right)=\bar{F}^{Z_{i}}\left(z_{i}\right)$ and $\operatorname{Bel}\left(Z_{i} \in\left(-\infty, z_{i}\right]\right)=F^{Z_{i}}\left(z_{i}\right), i=1,2$, respectively, of the model outputs $Z_{1}=Y_{1} \cdot Y_{2} \cdot Y_{3}$ (left) and $Z_{2}=$ $Y_{1} \cdot Y_{2} / Y_{3}$ (right) obtained by hybrid MC-FIA (solid lines) and MC-based DS-IRS (dashed lines) approaches

The results are very similar, i.e., in the present case, the effect of the different dependence relationships between the epistemically-uncertain paramenters is not evident. This is confirmed by the analysis of the corresponding quantitative indicators: actually, the intervals for $P\left[Z_{1}>z_{1}^{*}\right]$ and $Z_{1}^{0.95}$ are $[0.0013,0.0199]$ and $[617.55,868.93]$, respectively, in case (ii), whereas they are [0.0010, 0.0194] and [604.31, 867.44], respectively, in case (iii); in addition, the intervals for $P\left[Z_{2}>z_{2}^{*}\right]$ and $Z_{2}^{0.95}$ are $[0.0426$, $0.1944]$ and $[28.59,46.32]$, respectively, in case (ii), whereas they are [0.0436, 0.1728]

\footnotetext{
${ }^{g}$ Notice that the same conclusions could be drawn by the analysis of the other quantities of interest considered in the present paper, i.e., the 2.5-th and 97.5-th percentiles of the CDFs of the model outputs and the (upper and lower) CDFs of the 95-th quantile of the model outputs (see Sec. 3.3). A pictorial representation of such
} 
and [28.77, 44.71], respectively, in case (iii). Only for illustration purposes, a pictorial representation of the operative procedure which is adopted to identify the intervals for quantiles $Z_{1}^{0.95}$ and $Z_{2}^{0.95}$ is given in Fig. 2, left and right, respectively, with reference to the hybrid MC-FIA approach (solid lines). The intervals for the $95^{\text {th }}$ quantiles $Z_{1}^{0.95}$ and $Z_{2}^{0.95}$ of $Z_{1}$ and $Z_{2}$ are operatively identified by the arrows that originate at 0.95 on the ordinates of Fig. 2, extend horizontally on the upper and lower $\operatorname{CDFs} \bar{F}^{z_{i}}\left(z_{i}\right)$ and $\underline{F}^{z_{i}}\left(z_{i}\right), i=1,2$ (solid lines), and then drop vertically to the abscissas to produce the intervals $\left[\left(\bar{F}^{z_{1}}\right)^{-1}(0.95),\left(F^{z_{1}}\right)^{-1}(0.95)\right]=[617.55,868.93]$ and $\left[\left(\bar{F}^{z_{2}}\right)^{-1}(0.95),\left(F^{z_{2}}\right)^{-1}(0.95)\right]$ $=[28.59,46.32]$, respectively. The similarity between the results obtained the hybrid MC-FIA and MC-based DS-IRS approaches may be explained as follows. In the hybrid MC-FIA approach, the plausibility $\operatorname{Pl}(A)$ and belief $\operatorname{Bel}(A)$ functions of a given $\operatorname{set} A=(-$ $\infty, z]$ are calculated as $\int_{0}^{1} P l_{\alpha}(A) d \alpha$ and $\int_{0}^{1} B e l_{\alpha}(A) d \alpha$, respectively, i.e., as the integrals over $\alpha \in(0,1]$ of the different plausibility and belief functions, $P l_{\alpha}(A)$ and $\operatorname{Bel}_{\alpha}(A)$, respectively, obtained by fuzzy interval analysis at different possibility levels $\alpha \in(0,1]$ (see Sec. 2.2 and Appendix B for details). ${ }^{24,86,87}$ Instead, in the MC-based DS-IRS approach, $P l(A)$ and $B e l(A)$ can be computed as $P l(A)=\frac{1}{N_{e}} \sum_{i_{e}=1}^{N_{e}} P l_{i_{e}}(A)$ and $\operatorname{Bel}(A)=\frac{1}{N_{e}} \sum_{i_{e}=1}^{N_{e}} \operatorname{Bel}_{i_{e}}(A)$, respectively, i.e., as the arithmetic means of the different plausibility and belief functions $P l_{i_{e}}(A)$ and $B e l_{i_{e}}(A), i_{e}=1,2, \ldots, N_{e}$, obtained in correspondence of the $N_{e}$ different random combinations of the independent focal sets representing the epistemically-uncertain distribution parameters (see Sec. 2.3 and Appendix $\mathrm{C}$ for details). ${ }^{23,24}$ It is arguable that the different assumptions of (in)dependence between the epistemically-uncertain parameters affect the "distributions" of the plausibility and belief functions generated in correspondence of different "realizations" of epistemic uncertainty: in other words, the set of plausibility (resp., belief) functions $P l_{\alpha}(A)$ (resp., $\left.\operatorname{Bel}_{\alpha}(A)\right), \alpha \in(0,1]$, produced by the hybrid MC-FIA

quantities is not reported here for brevity sake; the corresponding quantitative indicators are summarized in Table 3. 
method (assuming total dependence) will be substantially different from the set $P l_{i_{e}}(A)$ (resp., $\left.\operatorname{Bel}_{i_{i}}(A)\right), i_{e}=1,2, \ldots, N_{e}$, generated by the MC-based DS-IRS approach (assuming independence). However, the differences in the "distributions" of such sets of functions may be averaged (i.e., in some cases cancelled out) when the synthetic indicators $P l(A)$ and $\operatorname{Bel}(A)$ are computed as integrals (in the hybrid MC-FIA method) or arithmetic means (in the MC-based DS-IRS approach) over the different "realizations" of epistemic uncertainty.

Then, in order to highlight the effects of the different assumptions about the (in)dependence relationships between the epistemically-uncertain parameters, the upper and lower CDFs, $\bar{F}^{z_{i}^{0.5}}\left(z_{i}^{0.95}\right)$ and $\underline{F}^{Z_{i}^{0.95}}\left(z_{i}^{0.95}\right)$, respectively, of the 95 -th quantile $Z_{i}^{0.95}$ of the model output $Z_{i}, i=1,2$, are further analyzed. Fig. 3 top shows the bounding CDFs for $Z_{1}^{0.95}$ (left) and $Z_{2}^{0.95}$ (right), produced by the hybrid MC-FIA (solid lines) and the MC-based DS-IRS (dashed lines) approach; for illustration purposes, Fig. 3 bottom shows the possibility distributions $\pi^{Z_{1}^{0.95}}\left(z_{1}^{0.95}\right)$ (left) and $\pi^{z_{2.9}^{0.5}}\left(z_{2}^{0.95}\right)$ (right) that correspond

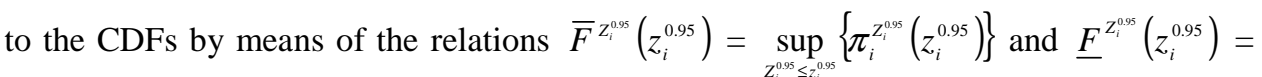
$1-\sup _{z_{i}^{005}>z_{i}^{0.95}}\left\{\pi_{i}^{z_{i}^{0.95}}\left(z_{i}^{0.95}\right)\right\}, i=1,2 . .^{23}$ 

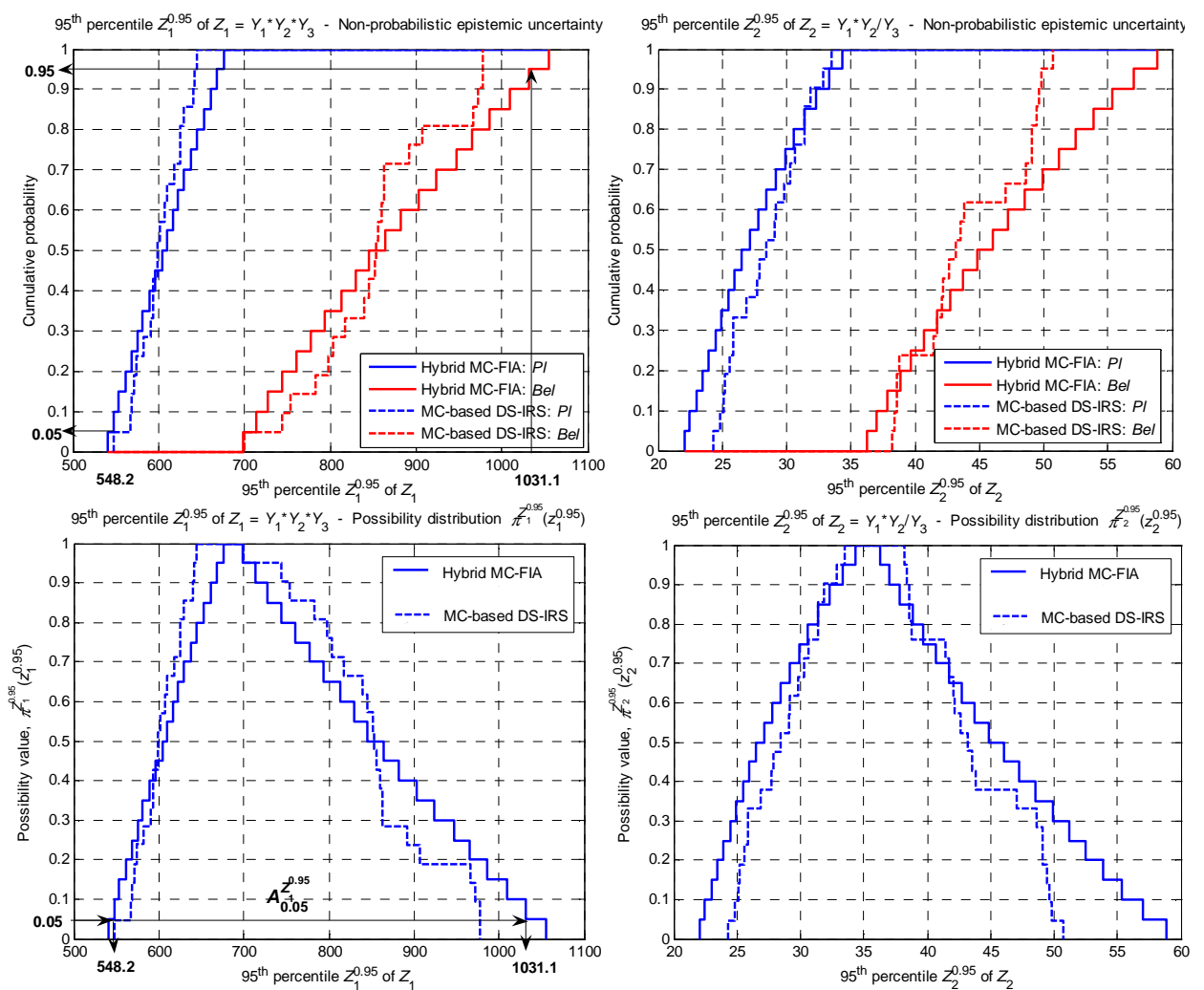

Fig. 3. Top: comparison of the upper and lower CDFs $\bar{F} Z_{i}^{0.95}\left(z_{i}^{0.95}\right)$ and $\underline{F}^{Z_{i}^{0.95}}\left(z_{i}^{0.95}\right)$ of the 95-th quantile $Z_{i}^{0.95}, i=1,2$, of the model outputs $Z_{1}=Y_{1} \cdot Y_{2} \cdot Y_{3}$ (left) and $Z_{2}=Y_{1} \cdot Y_{2} / Y_{3}$ (right) obtained by the hybrid MCFIA (solid lines) and MC-based DS-IRS (dashed lines) approaches. Bottom: possibility distributions
$\pi^{Z_{1}^{0.95}}\left(z_{1}^{0.95}\right)$ (left) and $\pi^{Z_{2}^{0.95}}\left(z_{2}^{0.95}\right)$ (right) of $Z_{z^{0.95}}^{0.95}$ and $Z_{2}^{0.95}$, respectively, corresponding to $\bar{F}_{i}^{0.95}\left(z_{i}^{0.95}\right)$ and $\underline{F}^{z_{i}^{0.95}}\left(z_{i}^{0.95}\right), i=1,2$

It can be seen that the hybrid MC-FIA method produces a larger gap between the upper and lower CDFs $\bar{F}^{z_{i}^{0.95}}\left(z_{i}^{0.95}\right)$ and $\underline{F}^{Z_{i}^{0.95}}\left(z_{i}^{0.95}\right)$ than the MC-based DS-IRS approach in the regions where the cumulative probabilities are close to "extreme" values, i.e., where $\bar{F}^{Z_{i}^{0.9}}\left(z_{i}^{0.95}\right) \approx 0$ and $\underline{F}^{Z_{i}^{0.95}}\left(z_{i}^{0.95}\right) \approx 1$. This is explained as follows. Notice that the values of $Z_{i}^{0.95}$ for which $\bar{F}^{Z_{i}^{0.95}}\left(z_{i}^{0.95}\right) \approx 0$ and $\underline{F}^{Z_{i}^{0.95}}\left(z_{i}^{0.95}\right) \approx 1$ correspond to the lower and upper bounds, respectively, of the $\alpha$-cut of level $\alpha \approx 0$ of the possibility distribution $\pi^{z_{i}^{z_{i}}}\left(z_{i}^{0.95}\right)$. For illustration purposes, by way of example the $\alpha$-cut $A_{0.05}^{Z_{105}^{095}}$ of level $\alpha=0.05$ of the possibility distribution $\pi^{Z_{1}^{0.95}}\left(z_{1}^{0.95}\right)$ produced by the hybrid MC-FIA is indicated by arrows in Fig. 3 bottom, left; the corresponding lower and upper bounds, i.e., 548.2 and 
1031.1, respectively, are shown to correspond to the cumulative probabilities $\underline{F}^{Z_{1}^{0.95}}(1031.1)=1-\sup _{Z_{1}^{0.9}>z_{1}^{0.95}}\left\{\pi_{1}^{Z_{1}^{0.95}}(1031.1)\right\}=1-\alpha=1-0.05=0.95$ and $\bar{F}^{Z_{1}^{0.95}}(548.2)=$ $\sup _{z_{1}^{0.95} \leq z_{1}^{0.95}}\left\{\pi_{1}^{Z_{1}^{0.95}}(548.2)\right\}=\alpha=0.05$ in Fig. 3 top, left. All this considered, it should be noticed that the $\alpha$-cut $A_{0}^{Z_{1}^{095}}$ of level $\alpha=0$ of the possibility distribution $\pi^{Z_{1}^{0.95}}\left(z_{1}^{0.95}\right)$ can be generated only by "combining" and propagating through the model function $Z_{1}=Y_{1} \cdot Y_{2} \cdot Y_{3}$ the $\alpha$-cuts of level $\alpha=0$ of all the possibilistic parameters $\mu_{1}=\theta_{1,1}, \sigma_{2}=\theta_{2,2}$ and $m=\theta_{3,2}$ of the model inputs $Y_{1}, Y_{2}$ and $Y_{3}$ (see Sec. 2.2 and Appendix B). Such combination of $\alpha$ values, i.e., $\left\{\alpha_{1}=0, \alpha_{2}=0, \alpha_{3}=0\right\}$, is always "processed" by fuzzy interval analysis in the hybrid MC-FIA method, due to the underlying assumption of total dependence between the information sources (e.g., the experts or observers) that supply the parameters possibility distributions: actually, the same possibility (resp. confidence) level $\alpha$ (resp., $1-\alpha$ ) is chosen to build the $\alpha$-cuts for all the epistemically-uncertain parameters (see Sec. 2.2 and Appendix B). On the contrary, such combination of possibility (resp., confidence) values, i.e., $\left\{\alpha_{1}=0, \alpha_{2}=0, \alpha_{3}=0\right\}$ (resp., $\left\{1-\alpha_{1}=1,1-\alpha_{2}=\right.$ $\left.1,1-\alpha_{3}=1\right\}$ ), cannot be obtained easily (i.e., with high probability) by the MC-based DSIRS approach, which performs a plain random sampling among independent intervals. This is coherent with the real processes of expert elicitation, in that it is difficult to find different (independent) experts that provide estimates about different uncertain parameters with the same (and, in this case, maximal) confidence.

The higher conservatism of the hybrid MC-FIA approach is reflected by the values of the quantitative indicators $P\left[Z_{i}^{0.95}>z_{i}^{0.95^{*}}\right]$ and $\left[Z_{i}^{0.95}\right]^{0.95}, i=1,2$, reported in Table 3 . For example, referring only to output $Z_{1}$ for brevity, it can be seen that $P\left[Z_{1}^{0.95}>Z_{1}^{0.95^{*}}\right]$ ranges within $[0,0.1500]$ for the hybrid MC-FIA method, whereas it is 0 for the MC-based DSIRS approach: thus, the assumption of independence between the epistemically-uncertain parameters leads to a dramatic underestimation of the exceedance probability. In addition, the quantile $\left[Z_{1}^{0.95}\right]^{0.95}$ ranges within $[668.52,1031.00]$ for the hybrid MC-FIAmethod, whereas it ranges within [641.80, 977.25] for the MC-based DS-IRS approach: again, the assumption of independence leads to underestimating the upper bound of the quantile by about $5.5 \%$. 
The same conclusions can be drawn by the analysis of the 2.5-th and 97.5-th percentiles $F^{Z_{i}, 0.025}\left(z_{i}\right)$ and $F^{Z_{i} 0.975}\left(z_{i}\right)$ of the CDF $F^{Z_{i}}\left(z_{i}\right)$ of $Z_{i}, i=1,2$ (Fig. 4, left and right). It can be seen that the CDFs produced by the hybrid MC-FIA approach (solid lines) completely envelop those produced by the MC-based DS-IRS method (dashed lines) (i.e., they represent more conservative estimates of the bounding distributions). As before, this is explained by the difficulty of plain MC simulation of randomly and independently sampling "extreme" (and more conservative) combinations of possibility (resp., confidence) levels $\alpha$ (resp., $1-\alpha$ ) when processing epistemic uncertainty.
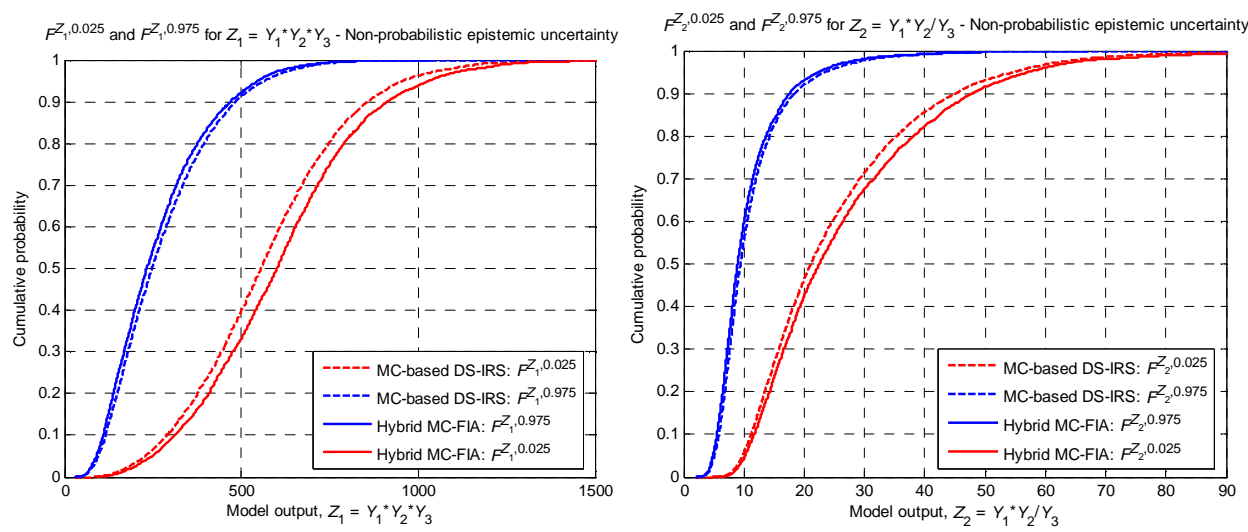

Fig. 4. Comparison of the 2.5-th and 97.5-th percentiles $F^{Z_{i}, 0.025}\left(z_{i}\right)$ and $F^{Z_{i}, 0.975}\left(z_{i}\right)$ of the CDF $F^{Z_{i}}\left(z_{i}\right)$, $i=1,2$, of the model outputs $Z_{1}=Y_{1} \cdot Y_{2} \cdot Y_{3}$ (left) and $Z_{2}=Y_{1} \cdot Y_{2} / Y_{3}$ (right) obtained by the hybrid MC-FIA (solid lines) and MC-based DS-IRS (dashed lines) approaches

Some considerations are in order with respect to the results obtained. The first comparison (between methods (i.a) and (i.b)) shows that the results produced by the double MC approach are strongly conditioned by the particular characteristics of the model function at hand (i.e., whether the function is increasing in all the variables or not, whether it is monotonic or not, ...): thus, different states of dependence between the epistemically-uncertain parameters of the input probability distributions produce completely different results also when applied to the same model function: for example, for model (1) (resp., (2)) independence (resp., total dependence) leads to less conservative results than total dependence (resp., independence). Thus, in a hypothetic risk assessment problem the analyst should know a priori the shape of the model function 
in order to guarantee conservatism. This raises serious concerns from the point of view of safety: actually, in the risk assessment of real safety-critical systems, most of the model functions adopted are not represented by explicit mathematical expressions, but rather by black boxes (i.e., implicit functions implemented in complex simulation codes). In such cases, the analyst must be aware of the fact that a probabilistic representation of epistemic uncertainty may fail to produce reliable and conservative results.

The second comparison (between methods (ii) and (iii)) shows instead that the state of dependence between the parameters is less critical when the representation of epistemic uncertainty is non-probabilistic: actually, the CDFs of the model outputs produced by the hybrid MC-FIA and the MC-based DS-IRS approaches are almost identical. However, the analysis of other quantitative indicators (e.g., the distribution of a given quantile of the output) shows that the hybrid MC-FIA method produces a larger separation between the plausibility and belief functions (i.e., more conservative results) than the MC-based DS-IRS approach (in particular, in the range of small probabilities that are of particular interest in the risk assessment of complex, highly reliable systems); in addition, contrarily to the double MC approach, the results produced by these methods do not seem to be affected by the characteristics of the model function at hand. Thus, in a non-probabilistic framework of epistemic uncertainty representation, the assumption of total dependence between the epistemically-uncertain parameters can be considered always more conservative than that of independence.

\subsubsection{Probabilistic/possibilistic representation of the epistemically-uncertain}

\section{distribution parameters}

In this Section, we perform comparisons between approaches (i.a) and (ii) and between approaches (i.b) and (iii), i.e., approaches that assume the same state of dependence between the epistemically-uncertain parameters, but represent epistemic uncertainty in different ways: in particular, in both hybrid MC-FIA (ii) and MC-based DS-IRS (iii) methods, possibility distributions are employed which identify a family of probability distributions for the epistemically-uncertain parameters; ${ }^{\text {h }}$ on the contrary, in the double MC approach ((i.a) and (i.b)), only a single probability distribution is assigned to represent the epistemic uncertainty associated to the parameters.

\footnotetext{
${ }^{\mathrm{h}}$ Recall that in the MC-based DS-IRS approach the possibility distributions are discretized into focal sets (see Sec. 2.3 and Appendix $\mathrm{C}$ for details).
} 
Fig. 5 shows the upper and lower Cumulative Distribution Functions (CDFs), $\bar{F}^{Z_{i}}\left(z_{i}\right)$ and $\underline{F}^{Z_{i}}\left(z_{i}\right)$, respectively, of the model outputs $Z_{1}=Y_{1} \cdot Y_{2} \cdot Y_{3}$ (left) and $Z_{2}=$ $Y_{1} \cdot Y_{2} / Y_{3}$ (right) obtained by the double MC approach assuming total dependence between the uncertain parameters (case (i.a), solid lines) and the plausibility and belief functions, $P l\left(Z_{i} \in\left(-\infty, z_{i}\right]\right)=\bar{F}^{Z_{i}}\left(z_{i}\right)$ and $\operatorname{Bel}\left(Z_{i} \in\left(-\infty, z_{i}\right]\right)=\underline{F}^{Z_{i}}\left(z_{i}\right), i=1,2$, respectively, produced by the hybrid MC-FIA approach (case (ii), dashed lines).
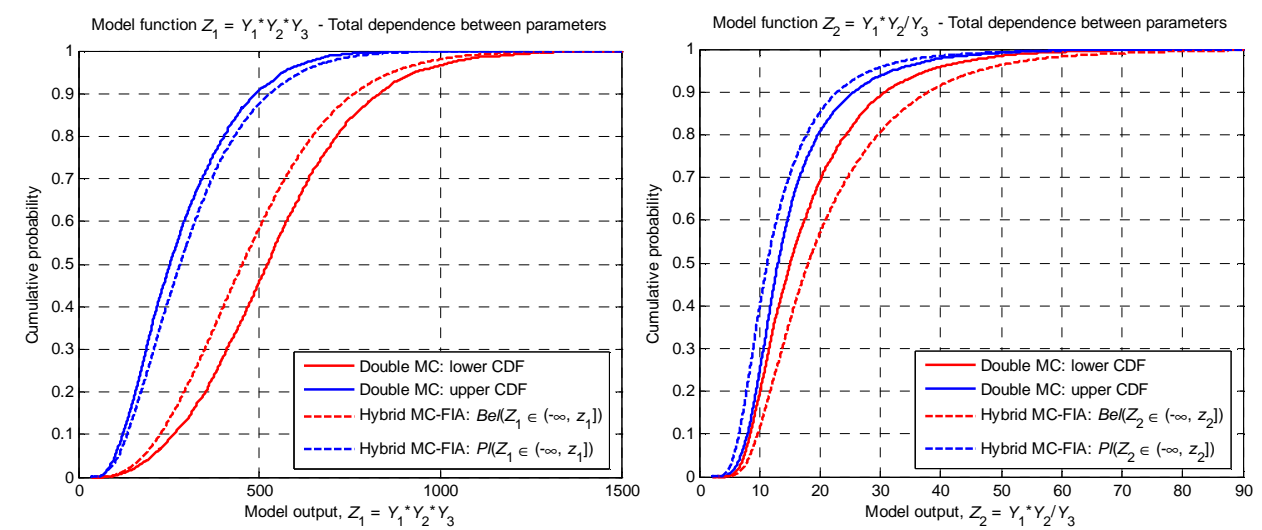

Fig. 5. Comparison of the upper and lower CDFs $\bar{F}^{Z_{i}}\left(z_{i}\right)$ and $\underline{F}^{Z_{i}}\left(z_{i}\right), i=1,2$, of the model outputs $Z_{1}=$ $Y_{1} \cdot Y_{2} \cdot Y_{3}$ (left) and $Z_{2}=Y_{1} \cdot Y_{2} / Y_{3}$ (right) obtained by a two-dimensional MC method considering total dependence between the epistemically-uncertain parameters (solid lines) and the hybrid MC-FIA approach (dashed lines)

It can be seen that for model function $Z_{1}=Y_{1} \cdot Y_{2} \cdot Y_{3}$ (1) (Fig. 5, left) the CDFs produced by the double MC method completely envelop those obtained by the hybrid MC-FIA approach. Referring to Sec. 4.1.1, these results can be explained as follows. The highest possible values for the model output $Z_{1}$ are obtained with a combination of high values of random variables $Y_{1}$ and $Y_{2}$ and $Y_{3}$ (which are favored on their turn by high values of the corresponding epistemically-uncertain parameters $\mu_{1}=\theta_{1,1}$ and $\sigma_{2}=\theta_{2,2}$ and $\left.m=\theta_{3,2}\right)$; conversely, the lowest possible values for the model output $Z_{1}$ are obtained with a combination of low values of $Y_{1}$ and $Y_{2}$ and $Y_{3}$ (which are favored on their turn by low values of the corresponding epistemically-uncertain parameters $\mu_{1}=\theta_{1,1}$ and $\sigma_{2}=\theta_{2,2}$ and $m=\theta_{3,2}$ ). In a double MC framework, these "extreme" situations (which give rise to the largest separation between the upper and lower cumulative distribution functions, i.e., to the most conservative case) are favored, i.e., are randomly sampled with high probability, 
when total dependence is assumed between the epistemically-uncertain parameters (see Sec. 4.1.1). Thus, it is very likely that the upper and lower CDFs produced by the double MC method assuming total dependence are obtained in correspondence of "extreme" combinations of epistemically-uncertain parameter values (i.e., combinations of values close to the extreme bounds of the ranges of variability of the epistemically-uncertain parameters). On the contrary, in the hybrid MC-FIA approach $\bar{F}^{z_{i}}\left(z_{i}\right)=$ $\operatorname{Pl}\left(Z_{i} \in\left(-\infty, z_{i}\right]\right)$ and $\underline{F}^{Z_{i}}\left(z_{i}\right)=\operatorname{Bel}\left(Z_{i} \in\left(-\infty, z_{i}\right]\right), i=1,2$, are obtained by averaging the different plausibility and belief functions (i.e., $P l_{\alpha}\left(Z_{i} \in\left(-\infty, z_{i}\right]\right)$ and $B e l_{\alpha}\left(Z_{i} \in(-\infty\right.$, $\left.z_{i}\right]$ ), respectively) generated at different possibility levels $\alpha \in(0,1]$ (in other words, by averaging the different contributions to the plausibility and belief functions produced by different $\alpha$-cuts of the epistemic parameters) (see Sec. 2.2 and Appendix B). Although this procedure is shown to provide the best bounds for the model outputs, ${ }^{24}$ it obviously prevents obtaining the "largest" possible bounds: actually, these extreme bounds are represented by $P l_{0}\left(Z_{i} \in\left(-\infty, z_{i}\right]\right)$ and $\operatorname{Bel}_{0}\left(Z_{i} \in\left(-\infty, z_{i}\right]\right)$, i.e., by the plausibility and belief functions generated in correspondence of the combination of the $\alpha$-cuts of level $\alpha=$ 0 (that are the largest possible).

The situation is reversed for model function $Z_{2}=Y_{1} \cdot Y_{2} / Y_{3}$ (2) (Fig. 5, left). Actually, in this case, the assumption of total dependence between the epistemically-uncertain parameters prevents the double MC method from obtaining conservative bounds because only a limited set of combinations of uncertain parameter values can be randomly explored (see the explanation above and Sec. 4.1.1).

As a final comparison, Fig. 6 shows the upper and lower CDFs, $\bar{F}^{z_{i}}\left(z_{i}\right)$ and $\underline{F}^{z_{i}}\left(z_{i}\right)$, $i=1,2$, respectively, of the model outputs $Z_{1}=Y_{1} \cdot Y_{2} \cdot Y_{3}$ (left) and $Z_{2}=Y_{1} \cdot Y_{2} / Y_{3}$ (right) obtained by the two-dimensional MC approach, considering independence between the epistemically-uncertain parameters (solid lines) and the MC-based DS-IRS approach (dashed lines). 

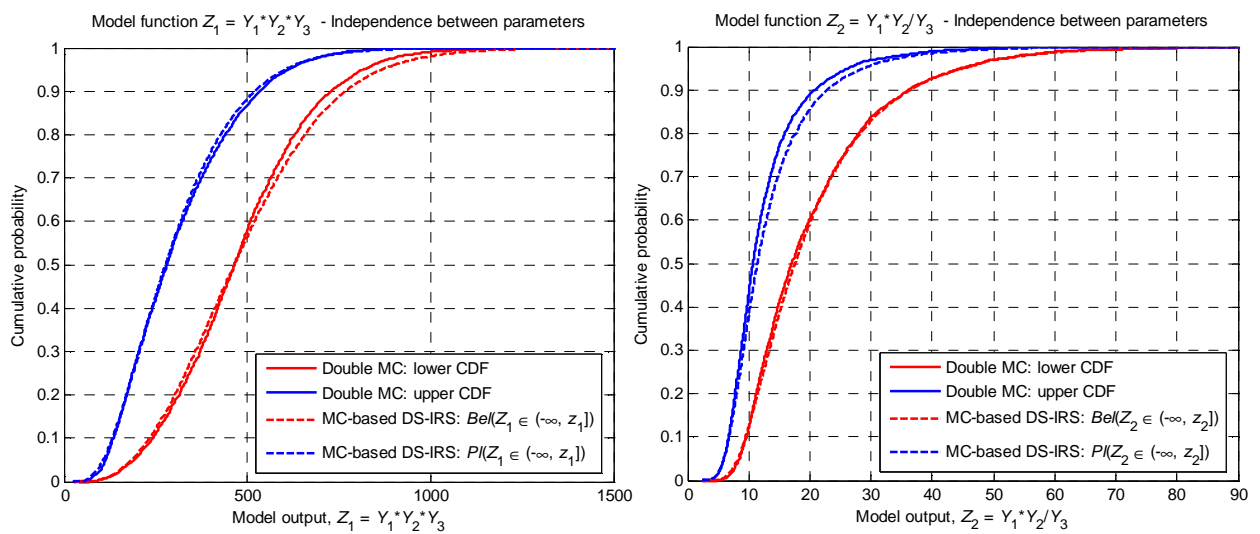

Fig. 6. Comparison of the upper and lower CDFs $\bar{F}^{Z_{i}}\left(z_{i}\right)$ and $\underline{F}^{Z_{i}}\left(z_{i}\right), i=1,2$, of the model outputs $Z_{1}=$ $Y_{1} \cdot Y_{2} \cdot Y_{3}$ (left) and $Z_{2}=Y_{1} \cdot Y_{2} / Y_{3}$ (right) obtained by the two-dimensional MC approach, considering independence between the epistemically-uncertain parameters (solid lines) and the MC-based DS-IRS approach (dashed lines)

The results are absolutely comparable: in particular, in the case of model function $Z_{1}$ $=Y_{1} \cdot Y_{2} \cdot Y_{3}$ (Fig. 6, left), the MC-based DS-IRS approach (dashed lines) is slightly more conservative than the double MC method (solid lines), whereas in the case of model function $Z_{2}=Y_{1} \cdot Y_{2} / Y_{3}$ (Fig. 6, right) the opposite situation occurs. This similarity can be explained by the common assumption of independence between the epistemicallyuncertain parameters and by the (similar) characteristics of the two algorithms used to propagate the uncertainties. In the MC-based DS-IRS approach, the focal sets generated by the discretization of the possibility distributions are selected randomly and independently by MC (step (2) of the procedure in Sec. 2.3 and Appendix C). Then, the minimum and maximum values of the model output of interest are identified letting the uncertain parameters range independently within the corresponding focal sets: thus, once the focal sets are selected, all possible combinations of parameter values can be explored, since the focal sets of all the parameters are exhaustively searched to maximize/minimize the model output. Similarly, in the double MC approach, a plain random sampling is performed from the probability distribution of the epistemically-uncertain parameters, which are considered independent: as a consequence of this independence, in principle all possible combinations of values of the parameters can be sampled, since the entire ranges of variability of the parameters are explored randomly and independently. 
In order to highlight the effects of different representations of epistemic uncertainty, the upper and lower CDFs, $\bar{F}_{i}^{z_{i}^{0.5}}\left(z_{i}^{0.95}\right)$ and $\underline{F}^{z_{i}^{095}}\left(z_{i}^{0.95}\right)$, respectively, of the 95-th quantile $Z_{i}^{0.95}$ of the output $Z_{i}, i=1,2$, are further analyzed. Fig. 7 shows the bounding CDFs $\bar{F}^{Z_{i}^{0.95}}\left(z_{i}^{0.95}\right)$ and $\underline{F}^{Z_{i}^{0.5}}\left(z_{i}^{0.95}\right)$ for $Z_{1}$ (left) and $Z_{2}$ (right), produced by the hybrid MC-FIA method (solid lines) together with the single $\mathrm{CDF} F^{z_{i}^{0.95}}\left(z_{i}^{0.95}\right)$ produced by the double MC method (assuming total dependence between the parameters) (dashed line); the corresponding quantities produced by the MC-based DS-IRS approach and the double MC method (assuming independence between the parameters) are not shown here for brevity sake.
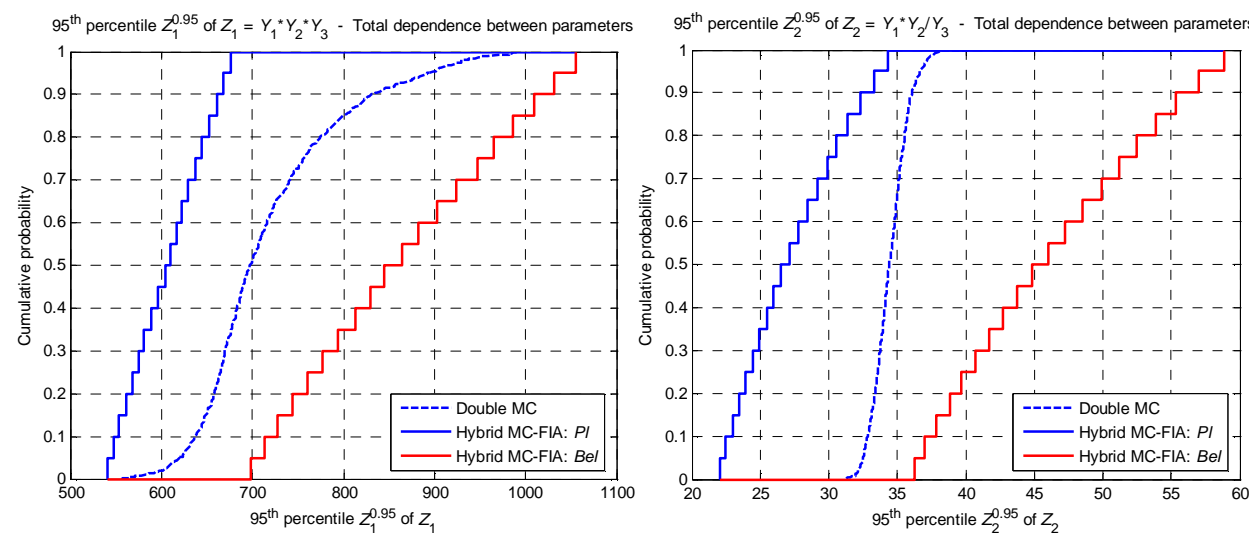

Fig. 7. Comparison of the upper and lower CDFs, $\bar{F}^{Z_{i}^{0.95}}\left(z_{i}^{0.95}\right)$ and $\underline{F}^{Z_{i}^{0.95}}\left(z_{i}^{0.95}\right)$, respectively, of the 95-th quantile $Z_{i}^{0.95}, i=1,2$, of the model outputs $Z_{1}=Y_{1} \cdot Y_{2} \cdot Y_{3}$ (left) and $Z_{2}=Y_{1} \cdot Y_{2} / Y_{3}$ (right) obtained by the double MC method assuming total dependence between the parameters (dashed lines) and the hybrid MC-FIA approach (solid lines)

Obviously, the advantage of using a non-probabilistic representation of epistemic uncertainty lies in the possibility of providing conservative bounds on the estimates of the 95-th quantile. For example, let us refer to the quantitative indicator $\left[Z_{2}^{0.95}\right]^{0.95}$ (Table 3): the point estimate provided by double MC is 36.65 , whereas the interval produced by the hybrid MC-FIA approach is [33.28, 57.02]. It can be seen that the upper bound of the interval [33.28, 57.02] produced by the hybrid MC-FIA approach overestimates by about $37.5 \%$ the corresponding point value generated by the double MC method. 
The higher conservatism of the hybrid MC-FIA approach is also evidenced by the analysis of the 2.5-th and 97.5-th percentiles $F^{Z_{i}, 0.025}\left(z_{i}\right)$ and $F^{Z_{i}, 0.975}\left(z_{i}\right)$ of the CDF $F^{Z_{i}}\left(z_{i}\right)$ of the model output $Z_{i}, i=1,2$. Fig. 8 shows the CDFs $F^{Z_{i}, 0.025}\left(z_{i}\right)$ and $F^{Z_{i}, 0.975}\left(z_{i}\right)$ for $Z_{1}$ (left) and $Z_{2}$ (right) produced by the double MC method assuming total dependence between the epistemically-uncertain parameters (dashed lines) and the hybrid MC-FIA approach (solid lines); as before, the corresponding quantities produced by the MC-based DS-IRS approach and the double MC method (assuming independence between the epistemically-uncertain parameters) are not shown here for brevity sake.
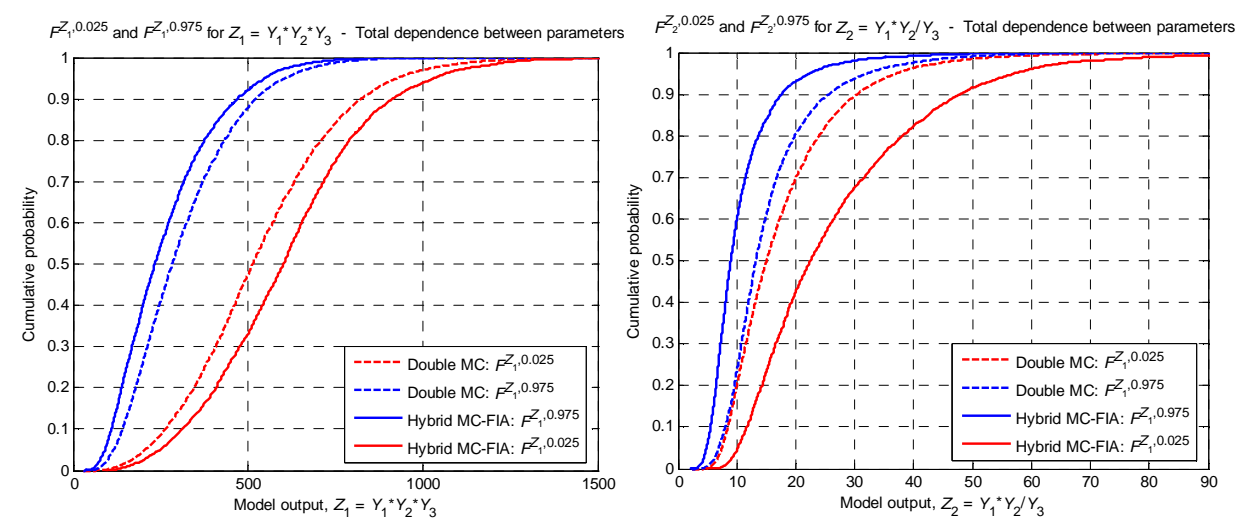

Fig. 8. Comparison of the 2.5-th and 97.5-th percentiles, $F^{Z_{i}, 0.025}\left(z_{i}\right)$ and $F^{Z_{i}, 0.975}\left(z_{i}\right)$, respectively, of the $\mathrm{CDF} F^{Z_{i}}\left(z_{i}\right), i=1,2$, of the model outputs $Z_{1}=Y_{1} \cdot Y_{2} \cdot Y_{3}$ (left) and $Z_{2}=Y_{1} \cdot Y_{2} / Y_{3}$ (right) obtained by the double MC method assuming total dependence between the parameters (dashed lines) and the hybrid MC-FIA approach (solid lines)

It can be seen that the CDFs produced by the hybrid method envelop those obtained by the purely probabilistic approach in all the cases considered. Particularly dramatic is the case of $Z_{2}=Y_{1} \cdot Y_{2} / Y_{3}$ (Fig. 8, right), where the gaps between the CDFs are impressively different. This is reflected by the values of the corresponding quantitative indicators (Table 3). For example, the estimates for the interval $\left[\left(F^{Z_{2}, 0.975}\right)^{-1}(0.95),\left(F^{Z_{2}, 0.025}\right)^{-1}(0.95)\right]$ of the 95-th quantile $Z_{2}^{0.95}$ are $[22.50,57.02]$ and [32.24, 37.15], for the hybrid MC-FIA and double MC approaches, respectively: thus, the width of the interval provided by the double MC method is 7 times smaller than that produced by the hybrid MC-FIA approach, which causes a serious underestimation of the 
quantile $Z_{2}^{0.95}$. In addition, the estimates for the interval $\left[F^{Z_{2}, 0.975}\left(z_{2}^{*}\right), F^{Z_{2}, 0.025}\left(z_{2}^{*}\right)\right]$ of $P\left[Z_{2}>z_{2}^{*}\right]$ are $[0.0190,0.3245]$ and $[0.0625,0.1050]$ for the MC-FIA and double MC approaches, respectively: again, the width of the interval provided by the double MC method is 7.2 times smaller than that produced by the hybrid approach, with a significant underestimation of the exceedance probability.

A final remark is in order with respect to the results obtained. The first comparison (between methods (i.a) and (ii)) shows that when there is total dependence between the epistemically-uncertain parameters, the effect of different representations of epistemic uncertainty on the conservatism of the results is not univocal, but rather it is related to the characteristics of the model function at hand. For example, in case of model function (1), a probabilistic representation of epistemic uncertainty provides a larger gap between the upper and lower CDFs of the model output (i.e., more conservative results) than a nonprobabilistic representation; on the contrary, for model function (2), the opposite situation occurs. As a consequence, embracing one representation of epistemic uncertainty instead of the other may significantly change the outcome of a decision making process in a risk assessment problem involving uncertainties: this is of paramount importance in systems that are critical from the safety view point, e.g., in the nuclear, aerospace, chemical and environmental fields.

The second comparison (between methods (i.b) and (iii)) shows instead that when there is independence between the epistemically-uncertain parameters, probabilistic and non-probabilistic representations of epistemic uncertainty produce absolutely comparable results: thus, in this case, embracing one representation of uncertainty instead of the other would not change significantly the final decision.

However, it is worth remembering that the considerations made above are valid if the analyst is interested only in the estimation of the upper and lower CDFs of the model output, but they do not hold in general for other quantities of interest in risk assessment (e.g., the distributions of a given quantile or the percentiles of the CDF of the model output). In these cases, a non-probabilistic representation of epistemic uncertainty always produces more reliable and conservative results than a probabilistic one, irrespective of (i) the state of dependence between the epistemically-uncertain parameters and (ii) the 
characteristics of the model function at hand. Therefore, even if the double MC approach purposedly tries to separate variability from imprecision, in many cases it fails to produce reliable and conservative results, which can raise great concerns from the safety point of view. This leads to conclude that when the state of dependence between the epistemically-uncertain parameters is not known to the analyst (which is far from unlikely in practice), a non-probabilistic representation of epistemic uncertainty may represent in most cases the "safest" choice.

Table 3. Values of the quantitative indicators of Sec. 3.3 produced by the double MC, hybrid MC-FIA and MC-based DS-IRS approaches in the joint hierarchical propagation of hybrid uncertainty through the model functions $Z_{1}=Y_{1} \cdot Y_{2} \cdot Y_{3}(1)$ and $Z_{2}=Y_{1} \cdot Y_{2} / Y_{3}(2)$ of Sec. 3.1

\begin{tabular}{|c|c|c|c|c|c|}
\hline & & \multicolumn{4}{|c|}{$Z_{1}=Y_{1} \cdot Y_{2} \cdot Y_{3}$} \\
\hline & & & Me & ods & \\
\hline \multicolumn{2}{|c|}{ Quantitative indicators } & $\begin{array}{c}\text { Double MC } \\
\text { (dep.) }\end{array}$ & $\begin{array}{c}\text { Double MC } \\
\text { (indep.) }\end{array}$ & MC-FIA & $\begin{array}{c}\text { MC-based } \\
\text { DS-IRS }\end{array}$ \\
\hline \multirow{2}{*}{$\overline{\boldsymbol{F}}^{z_{i}}, \underline{\boldsymbol{F}}^{z_{i}}$} & $P\left(Z_{1}>z_{1}{ }^{*}\right)$ & {$[0.0006,0.0342]$} & {$[0.0004,0.0092]$} & {$[0.0013,0.0199]$} & {$[0.0010,0.0194]$} \\
\hline & $Z_{1}^{0.95}$ & {$[566.86,932.13]$} & {$[613.58,816.07]$} & {$[617.55,868.93]$} & {$[604.31,867.44]$} \\
\hline \multirow{2}{*}{$\begin{array}{l}\boldsymbol{F}^{z_{1}, 0.025}\left(z_{1}\right), \\
\boldsymbol{F}^{z_{1}, 0.975}\left(z_{1}\right)\end{array}$} & $P\left(Z_{1}>z_{1}{ }^{*}\right)$ & {$[0.0006,0.0305]$} & {$[0.0010,0.0125]$} & {$[0.0002,0.0611]$} & {$[0.000,0.0378]$} \\
\hline & $Z_{1}^{0.95}$ & {$[606.20,925.50]$} & {$[633.45,823.70]$} & {$[548.10,1031.00]$} & {$[567.40,977.25]$} \\
\hline \multirow{2}{*}{$\overline{\boldsymbol{F}}^{z_{i}^{a s s}, \underline{\boldsymbol{F}}^{z_{i s s}^{a s s}}}$} & $P\left(Z_{1}^{0.95}>z_{1}{ }^{0.95 *}\right)$ & 0.000 & 0.000 & {$[0.000,0.1500]$} & {$[0.000,0.000]$} \\
\hline & {$\left[Z_{1}{ }^{0.95}\right]^{0.95}$} & 892.61 & 809.45 & {$[668.52,1031.00]$} & {$[641.80,977.25]$} \\
\hline & & \multicolumn{4}{|c|}{$Z_{2}=Y_{1} \cdot Y_{2} / Y_{3}$} \\
\hline & & \multicolumn{4}{|c|}{ Methods } \\
\hline \multicolumn{2}{|c|}{ Quantitative indicators } & $\begin{array}{c}\text { Double MC } \\
\text { (dep.) }\end{array}$ & $\begin{array}{l}\text { Double MC } \\
\text { (indep.) }\end{array}$ & MC-FIA & $\begin{array}{c}\text { MC-based } \\
\text { DS-IRS }\end{array}$ \\
\hline \multirow{2}{*}{$\overline{\boldsymbol{F}}^{z_{t}}, \underline{\boldsymbol{F}}^{z_{t}}$} & $P\left(Z_{2}>z_{2}{ }^{*}\right)$ & {$[0.0626,0.1108]$} & {$[0.0318,0.1640]$} & {$[0.0426,0.1944]$} & {$[0.0436,0.1728]$} \\
\hline & $Z_{2}^{0.95}$ & {$[32.12,38.05]$} & {$[26.36,45.03]$} & {$[28.59,46.32]$} & {$[28.77,44.71]$} \\
\hline \multirow{2}{*}{$\begin{array}{c}\boldsymbol{F}^{z_{1}, 0.025}\left(z_{1}\right) \\
\boldsymbol{F}^{z_{1}, 0.975}\left(z_{1}\right)\end{array}$} & $P\left(Z_{2}>z_{2}{ }^{*}\right)$ & {$[0.0625,0.1050]$} & {$[0.0385,0.1639]$} & {$[0.0190,0.3245]$} & {$[0.0225,0.2850]$} \\
\hline & $Z_{2}^{0.95}$ & {$[32.24,37.15]$} & {$[27.69,43.25]$} & {$[22.50,57.02]$} & {$[23.54,49.84]$} \\
\hline \multirow{2}{*}{$\overline{\boldsymbol{F}}^{z_{i}^{a s s}}, \underline{\boldsymbol{F}}^{z_{i s s}^{s, s}}$} & $P\left(Z_{2}^{0.95}>z_{2}^{0.95 *}\right)$ & 0.000 & 0.000 & {$[0.000,0.1500]$} & {$[0.000,0.000]$} \\
\hline & {$\left[Z_{2}^{0.95}\right]^{0.95}$} & 36.65 & 41.76 & {$[33.28,57.02]$} & {$[32.85,49.84]$} \\
\hline
\end{tabular}

\subsection{Inclusion of unknown dependences between the aleatory variables in a "level- 2" framework}

In this Section, the Dependency Bound Convolution (DBC) method ${ }^{69}$ is framed within a "level-2" setting of hybrid (i.e., probabilistic and possibilistic) uncertainty propagation: this allows accounting for all the (possibly unknown) dependences that may exist between the aleatory variables $Y_{1}, Y_{2}$ and $Y_{3}$ (i.e., the inputs to model functions $Z_{1}=$ $Y_{1} \cdot Y_{2} \cdot Y_{3}(1)$ and $Z_{2}=Y_{1} \cdot Y_{2} / Y_{3}(2)$ of Sec. 3.1). 
Fig. 9 shows the upper and lower CDFs $\bar{F}^{z_{i}}\left(z_{i}\right)$ and $\underline{F}^{Z_{i}}\left(z_{i}\right), i=1,2$, of the model outputs $Z_{1}$ (left) and $Z_{2}$ (right) obtained by the hybrid MC-FIA (dashed lines, Sec. 2.2 and Appendix B) and DBC-FIA (solid lines, Appendix D) approaches, which assume independence and unknown dependence, respectively, between the aleatory variables $Y_{1}$, $Y_{2}$ and $Y_{3}$.
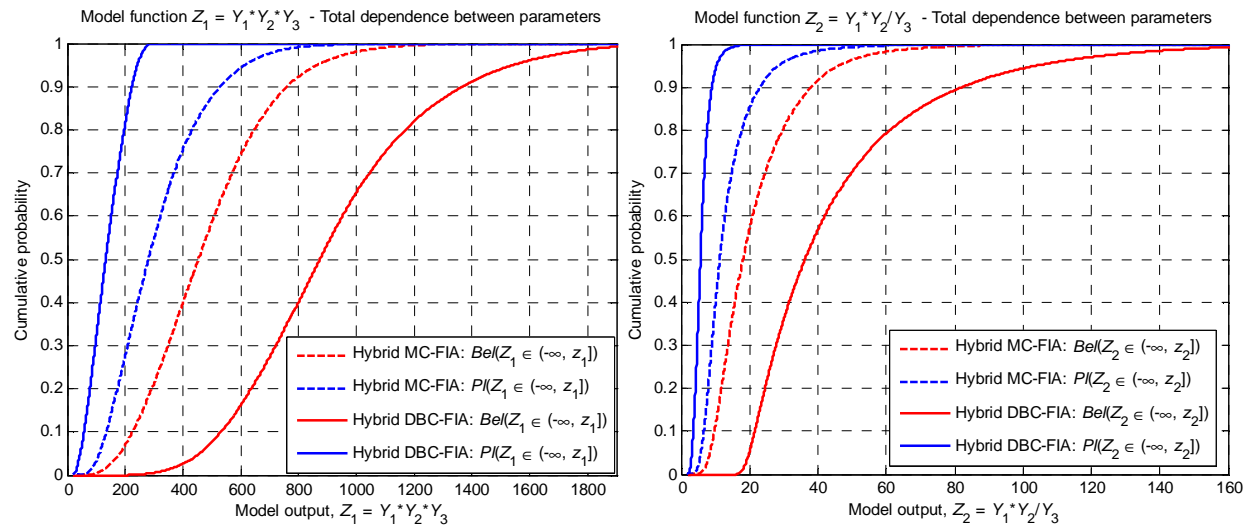

Fig. 9. Comparison of the upper and lower CDFs, $\bar{F}^{Z_{i}}\left(z_{i}\right)$ and $\underline{F}^{Z_{i}}\left(z_{i}\right), i=1,2$, respectively, of the model outputs $Z_{1}=Y_{1} \cdot Y_{2} \cdot Y_{3}$ (left) and $Z_{2}=Y_{1} \cdot Y_{2} / Y_{3}$ (right) obtained by the hybrid MC-FIA (dashed lined) and hybrid DBC-FIA (solid lines) approaches, assuming independence and unknown dependence, respectively, between the aleatory variables $Y_{1}, Y_{2}$ and $Y_{3}$

As expected, the inclusion of all kinds of possible dependences between the aleatory variables increases significantly the gap between the upper and lower CDFs of the model outputs (and, correspondingly, the conservatism of the results). This is confirmed by the analysis of the quantitative indicators $P\left[Z_{i}>z_{i}^{*}\right]$ and $Z_{i}^{0.95}, i=1,2$. For example, the intervals for $P\left[Z_{1}>Z_{1}^{*}\right]$ and $Z_{1}^{0.95}$ are $[0.0013,0.0199]$ and $[617.55,868.93]$, respectively, in case of independence, whereas they are [0, 0.3460] and [242.36, 1547.23], respectively, in case of unknown dependence: thus, the assumption of independence leads to underestimating the (upper bound of the) exceedance probability and the quantile by about 17 and 2 times, respectively. In addition, the intervals for $P\left[Z_{2}>z_{2}^{*}\right]$ and $Z_{2}^{0.95}$ are $[0.0426,0.1944]$ and $[28.59,46.32]$, respectively, in case of independence, whereas they are [0,0.5249] and [10.40, 104.15], respectively, in case of unknown dependence: again, the assumption of independence leads to underestimating 
the (upper bound of the) exceedance probability and the quantile by about 3 and 2.3 times, respectively.

Based on the results above, it can be concluded that the use of the DBC approach within a "level-2" setting may be very useful to provide an initial "worst-case" estimate of the risk associated to the system at hand when nothing is known about the real state of dependence between the input variables; however, in many realistic applications this would lead to excessively conservative (and thus pessimistic) results that would need to be refined by acquiring knowledge on the system and, in particular, on the actual state of dependence of the random variables of the model.

\section{Discussion and conclusions}

In the present paper, the two-dimensional MC, hybrid MC-FIA and MC-based DS-IRS approaches have been considered for the joint hierarchical propagation of hybrid (probabilistic and possibilistic) uncertainty within a "level-2" framework. Two examples have been taken as reference. Two issues have been addressed in the analyses:

(i) the implicit assumptions about the (in)dependence relationships among parameters subject to epistemic uncertainty (e.g., the hybrid MC-FIA approach assumes total dependence, whereas the MC-based DS-IRS method assumes random set independence);

(ii) the use of standard MC sampling to propagate the aleatory uncertainties, which implicitly assumes independence between the random variables.

With respect to issue (i) above, the two-dimensional MC, hybrid MC-FIA and MCbased DS-IRS approaches have been compared with the following objectives:

(a) the study of the effects of dependence between the epistemically-uncertain parameters of the aleatory probability distributions when a probabilistic/nonprobabilistic representation of epistemic uncertainty is adopted:

- the comparison between two-dimensional MC approaches assuming total dependence and independence between the epistemically-uncertain parameters, respectively, has shown that the results produced by the double MC approach are strongly related to the particular characteristics of the model function at hand (i.e., whether the function is increasing in all the variables or not, whether it is monotonic or not, ...): thus, different states of dependence between the epistemically-uncertain parameters of the input probability distributions may 
give rise to completely different results also when applied to the same model function. In particular, when the output is increasing in each place with respect to the inputs (e.g., the model function contains only products), assuming total dependence between the epistemically-uncertain parameters leads to a larger gap between the upper and lower CDFs of the model output (i.e., to more conservative results); on the contrary, when the output is not increasing in each place with respect to the inputs (e.g., the model function contains both products and quotients), the opposite situation occurs: the assumption of total dependence typically produces a consistently smaller gap between the bounding CDFs of the model output (i.e., less conservative results);

- the comparison between hybrid MC-FIA and MC-based DS-IRS approaches has shown that the plausibility and belief functions of the model output produced by the two approaches are similar: in other words, the computation of the upper and lower CDFs of the output is not significantly influenced by the different assumptions of (in)dependence between the epistemically-uncertain parameters. This is due to the fact that the different CDFs generated in correspondence of different (dependent or independent) "realizations" of epistemic uncertainty by the hybrid MC-FIA and MC-based DS-IRS approaches, respectively, are averaged to obtain the plausibility and belief functions of the model output: such procedure typically "cancels out" the effect of the particular state of dependence between the epistemically-uncertain parameters. However, this is not the case for other quantities of interest in risk assessment, e.g., the distributions of a given quantile or the percentiles of the CDF of the model output: in these cases, the hybrid MC-FIA method produces more conservative results than the MCbased DS-IRS approach. Actually, in processing epistemic uncertainty the assumption of total dependence allows selecting "extreme" combinations of $\alpha$ cuts (e.g., the combination of all the $\alpha$-cuts with possibility level $\alpha=0$ ) that cannot be easily obtained by plain random sampling of independent sets: this produces conservative estimates, in particular in the range of extreme cumulative probabilities (i.e., around 0 and 1).

- contrarily to probabilistic approaches, the results produced by hybrid methods do not seem to be affected by the characteristics of the model function at hand. 
Based on the considerations above, it can be concluded that:

- when the representation of epistemic uncertainty is probabilistic, the state of dependence between the epistemically-uncertain parameters of the aleatory probability distributions becomes a critical factor in risk-informed decisions because the effect of different (in)dependence assumptions on the conservatism of the estimates is closely related to the structure of the model function at hand. This raises serious concerns from the point of view of safety: actually, in the risk assessment of real safety-critical systems, many of the model functions adopted are not represented by explicit mathematical expressions, but rather by black boxes (i.e., implicit functions implemented in complex simulation codes). In such cases, two options are suggested: (1) the analyst performs a sensitivity study to gather the largest amount possible of information about the characteristics of the model function at hand; on the basis of the indications obtained, he/she "artificially" selects the state of the dependence between the epistemicallyuncertain parameters that produces the most conservative results; (2) the analyst assumes independence between the epistemically-uncertain parameters, which has been shown to produce more conservative results than total dependence when the model function is not increasing in each place with respect to the inputs;

- when the representation of the epistemically-uncertain parameters is nonprobabilistic, the state of dependence between the epistemically-uncertain parameters of the aleatory probability distributions is less critical. However, the hybrid MC-FIA method may be preferred to the MC-based DS-IRS approach because it provides more conservative results in the estimation of (i) the distributions of a given quantile of the model output and (ii) the percentiles of the CDF of the output. In addition, this higher conservatism is particularly evident in the range of extreme probabilities (i.e., around 0 and 1) that are of paramount importance in realistic risk assessment applications involving complex, highly reliable engineering systems.

(b) the study of the effect of the probabilistic/non-probabilistic representation of epistemic uncertainty when the state of dependence between the epistemicallyuncertain parameters is defined: 
- the comparison between the MC-based DS-IRS approach and the twodimensional MC approach assuming independence between the epistemicallyuncertain parameters has shown that in the case studies considered the upper and lower CDFs of the model output produced by the two approaches are similar. This is due to (i) the common assumption of independence between the epistemically-uncertain parameters; (ii) the similar characteristics of the two algorithms used to propagate the uncertainties and (iii) the fact that the computation of the bounding CDFs of the model output in the double MC approach is strongly influenced by the occasional random sampling of "extreme" combinations of values of the epistemically-uncertain parameters;

- the comparison between the hybrid MC-FIA method and the two-dimensional MC approach assuming total dependence between the epistemically-uncertain parameters has shown that the conservatism of the results depends on the structure of the model function at hand. In particular, when the model function is not increasing in each place with respect to the inputs (e.g., it contains both multiplications and quotients), the gap between the plausibility and belief functions of the output produced by the hybrid approach is typically larger than the gap between the upper and lower CDFs produced by the two-dimensional MC method. This is explained by the fact that in the two-dimensional MC approach the assumption of total dependence prevents the random sampling of "extreme" combinations of epistemically-uncertain parameters. On the contrary, in the hybrid MC-FIA method, an exhaustive interval analysis is performed for different $\alpha$-cuts of the possibility distributions: the result is that the hybrid approach is able to explore a larger set of combinations of epistemicallyuncertain parameter values than the double MC approach, thus producing more conservative results.

Instead, when the model function is increasing in each place with respect to the inputs (e.g., it contains only multiplications), the opposite situation occurs: actually, in this case the two-dimensional MC approach assuming total dependence is very likely to sample "extreme" combinations of the epistemically-uncertain parameters; 
- both non-probabilistic approaches (i.e., the hybrid MC-FIA and the MC-based DS-IRS methods) always lead to more conservative results than the probabilistic approaches (i.e., the two-dimensional MC method assuming total dependence and independence) in the estimation of quantities like the distribution of a quantile of the output or the percentiles of the CDFs of the output (i.e., those quantities whose computation is not influenced by the occasional random sampling of "extreme" combinations of values of the epistemically-uncertain parameters). In particular, (i) the non-probabilistic approaches are able to produce upper and lower distributions for all the quantiles of the output, whereas the two-dimensional MC method provides only a single probability distribution; (ii) the percentiles of the CDFs of the output produced by the non-probabilistic approaches completely envelop those generated by the probabilistic approaches.

Based on the considerations above, it can be concluded that:

- if the analyst is interested only in the estimation of the upper and lower CDFs of the model output:

o when there is total dependence between the epistemically-uncertain parameters, a probabilistic representation of the epistemically-uncertain parameters of the aleatory probability distributions may fail to produce reliable and conservative results, which raises concerns from the point of view of safety;

o when there is independence between the epistemically-uncertain parameters, both probabilistic and non-probabilistic representations of the epistemically-uncertain parameters may be chosen since they may (occasionally) produce comparable results;

- if the analyst is interested in the estimation of quantities like the distribution of a given quantile or the percentiles of the CDFs of the output, a non-probabilistic representation of epistemic uncertainty is in general suggested because it provides more conservative results.

The findings of the comparison show that adopting different methods for jointly propagating aleatory and epistemic uncertainties may generate different results and possibly different decisions in risk problems involving uncertainties: this is of paramount importance in systems that are critical from the safety viewpoint, e.g., in the nuclear, 
aerospace, chemical and environmental fields. In particular, it seems advisable to suggest that, if nothing is known about the dependence relationship between the epistemicallyuncertain parameters, one should resort to the hybrid MC-FIA approach because its risk estimates are more conservative than (or at least comparable to) those obtained by the double MC approach assuming dependence (or independence) between the epistemicallyuncertain parameters: thus, a non-probabilistic representation of epistemic uncertainty represents in general a "safer" choice.

With respect to issue (ii) above, the DBC method has been framed for the first time within a "level-2" setting of hybrid uncertainty propagation with the objectives of: (a) removing the assumption of independence between the aleatory variables (which is implicit in the adoption of standard MC sampling for the propagation of the aleatory uncertainties) and (b) accounting for all kinds of (possibly unknown) dependences between the aleatory variables, i.e., also those that cannot be modeled even within arbitrarily complex MC sampling frameworks (e.g., copulas). It has been shown that the upper and lower CDFs of the output produced by the hybrid DBC-FIA approach completely envelop those obtained by the hybrid MC-FIA method. Based on the results obtained, it can be concluded that the use of the DBC approach within a "level-2" setting may be very useful to provide an initial "worst-case" estimate of the risk associated to the system at hand when nothing is known about the real state of dependence between the variables; however, in many realistic applications this would lead to excessively conservative (and thus pessimistic) results that need to be refined by acquiring further knowledge on the system, its model and the real state of dependence between the random variables.

The findings and conclusions drawn by the comparisons performed in Sec. 4 are summarized in Table 4 for the sake of clarity. 
Table 4. Comparisons performed in Sec. 4, and their relative findings

\begin{tabular}{|c|c|c|c|}
\hline \multicolumn{4}{|c|}{ Comparison between the uncertainty propagation methods (Sec. 4.1) } \\
\hline \multicolumn{4}{|c|}{ Sec. 4.1.1 } \\
\hline & & \multicolumn{2}{|c|}{ Representation of epistemic uncertainty } \\
\hline & & Probabilistic & Non-probabilistic \\
\hline \multirow{4}{*}{$\begin{array}{l}\text { State of epistemic } \\
\text { dependence }\end{array}$} & Independence & Double MC (i.b.) & MC-based DS-IRS (iii.) \\
\hline & & vs & vs \\
\hline & $\begin{array}{c}\text { Total } \\
\text { dependence }\end{array}$ & Double MC (i.a.) & Hybrid MC-FIA (ii.) \\
\hline & & $\downarrow$ & $\downarrow$ \\
\hline & Findings & \multicolumn{2}{|c|}{$\begin{array}{l}\text { Method (i.a) vs (i.b): } \\
\text { The state of dependence between the parameters is critical because } \\
\text { its effect on the conservatism of the estimates is related to the } \\
\text { structure of the model function } \\
\text {-Two options are suggested: } 1 \text { ) perform a sensitivity study to get } \\
\text { information about the model function and "artificially" select the state } \\
\text { of dependence that produces the most conservative results; } 2 \text { ) assume } \\
\text { independence between the parameters, which is more conservative } \\
\text { than total dependence when the model function is not increasing in } \\
\text { each place with the inputs } \\
\text { Method (ii) vs (iii): } \\
\text {-The state of dependence is not so critical (e.g., it has almost no effect } \\
\text { on the upper and lower CDFs of the model output) } \\
\text {-Hybrid MC-FIA may be preferred to MC-based DS-IRS because it is } \\
\text { more conservative in the estimation of i) the distributions of a given } \\
\text { quantile of the model output and ii) the percentiles of the CDF of the } \\
\text { output (in particular, in the range of extreme probabilities, i.e., around } \\
0 \text { and 1) }\end{array}$} \\
\hline \multicolumn{4}{|c|}{ Sec. 4.1.2 } \\
\hline & & \multicolumn{2}{|c|}{ State of epistemic dependence } \\
\hline & & Independence & Total dependence \\
\hline \multirow{4}{*}{$\begin{array}{l}\text { Representation of } \\
\text { epistemic } \\
\text { uncertainty }\end{array}$} & Probabilistic & Double MC (i.b.) & Double MC (i.a.) \\
\hline & & vs & vs \\
\hline & $\begin{array}{c}\text { Non- } \\
\text { probabilistic }\end{array}$ & MC-based DS-IRS (iii.) & Hybrid MC-FIA (ii.) \\
\hline & & $\downarrow$ & $\downarrow$ \\
\hline & Findings & \multicolumn{2}{|c|}{$\begin{array}{l}\text { General: } \\
\text {-In the estimation of quantities like the distribution of a given quantile } \\
\text { or the percentiles of the CDFs of the output, a non-probabilistic } \\
\text { representation of epistemic uncertainty is suggested because it } \\
\text { provides more conservative results } \\
\text { Method (i.b.) vs (iii): } \\
\text {-In the estimation of the upper and lower CDFs of the output, both } \\
\text { representations of epistemic uncertainty may be chosen since they } \\
\text { may (occasionally) produce comparable results } \\
\text { Method (i.a.) vs }(i i) \text { : } \\
\text {-In the estimation of the upper and lower CDFs of the output, a } \\
\text { probabilistic representation of epistemic uncertainty may fail to } \\
\text { produce reliable and conservative results }\end{array}$} \\
\hline
\end{tabular}


(Table 4. Continued).

\begin{tabular}{|c||c|c|c|}
\hline \multicolumn{3}{|c|}{ Unknown dependences between aleatory variables by DBC (Sec. 4.2) } \\
\hline \multicolumn{1}{|c||}{ State of dependence between the aleatory variables } \\
\hline Independence & & Unknown dependence \\
\hline Findings & Hybrid MC-FIA (ii.) & vs & Hybrid DBC-FIA (iv.) \\
\hline & $\begin{array}{l}\text {-The upper and lower CDF of the output produced by hybrid DBC-FIA } \\
\text { completely envelop those obtained by hybrid MC-FIA }\end{array}$ \\
$\begin{array}{l}\text { - Hybrid DBC-FIA is useful to provide an initial "worst-case" estimate } \\
\text { of risk when nothing is known about the real state of dependence } \\
\text { between the random variables } \\
\text { - In realistic applications hybrid DBC-FIA leads to excessively } \\
\text { conservative (and thus pessimistic) results that need to be refined by } \\
\text { acquiring further knowledge on the system, its model and the real state } \\
\text { of dependence between the random variables }\end{array}$ \\
\hline
\end{tabular}

\section{References}

1. USNRC, Guidance on the Treatment of Uncertainties Associated with PRAs in RiskInformed Decision Making. NUREG-1855, US Nuclear Regulatory Commission (Washington, DC, 2009).

2. NASA, Risk-Informed Decision Making Handbook, NASA/SP-2010-576 - Version 1.0 (April 2010).

3. G.E. Apostolakis, The concept of probability in safety assessment of technological systems, Science 250 (1990) 1359-1364.

4. J.C. Helton and W. Oberkampf, Alternative representations of epistemic uncertainties. Reliability Engineering and System Safety 85(1-3) (2004) 1-10.

5. P. Limbourg, E. de Rocquigny, Uncertainty analysis using evidence theory - confronting level-1 and level-2 approaches with data availability and computational constraints, Reliability Engineering and System Safety 95(5) (2010) 550-564.

6. NUREG-CR-6850, EPRI/NRC-RES Fire PRA methodology for nuclear power facilities, Volume 2: detailed methodology. US Nuclear Regulatory Commission (Washington, DC 2005).

7. G.E. Apostolakis, S. Kaplan, Pitfalls in risk calculations, Reliability Engineering 2(2) (1981) 135-145.

8. D. Huang, T. Chen, M.J. Wang, A fuzzy set approach for event tree analysis. Fuzzy Sets and Systems 118 (2001) 153-165.

9. USNRC, An approach for using probabilistic risk assessment in risk-informed decisions on plant-specific changes to the licensing basis. NUREG-1.174 - Revision 1 (US Nuclear Regulatory Commission, Washington, DC, 2002).

10. T. Aven, On the Need for Restricting the Probabilistic Analysis in Risk Assessments to Variability, Risk Analysis 30(3) (2010) 354-360.

11. T. Aven, Interpretations of alternative uncertainty representations in a reliability and risk analysis context, Reliability Engineering \& System Safety 96(3) (2011) 353-360.

12. T. Aven and R. Steen, The concept of ignorance in a risk assessment and risk management context, Reliability Engineering and System Safety 95(11) (2010) 11171122 .

13. T. Aven, E. Zio, Some considerations on the treatment of uncertainties in risk assessment for practical decision making, Reliability Engineering and System Safety 96(1) (2010) 6474. 
14. S. Ferson, L. Ginzburg, R. Akcakaya, Whereof one cannot speak: when input distributions are unknown, Risk Analysis, 1996.

15. G.J. Klir, B. Yuan, Fuzzy Sets and Fuzzy Logic: Theory and Applications (Prentice-Hall, Upper Saddle River, NJ, 1995).

16. S. Ferson, V. Kreinovich, L. Ginzburg, K. Sentz, D.S. Myers, Constructing probability boxes and Dempster-Shafer structures, Sandia National Laboratories, Technical Report SAND2002-4015 (Albuquerque, New Mexico, 2003).

17. S. Ferson, R.B. Nelsen, J. Hajagos, D.J. Berleant, J. Zhang, W.T. Tucker, L.R. Ginzburg, W.L. Oberkampf, Dependence in probabilistic modeling, Dempster-Shafer theory, and probability bounds analysis, Technical Report SAND2004-3072 (Albuquerque, New Mexico, 2004).

18. J.C. Helton, J.D. Johnson, W.L. Oberkampf, C.B. Storlie, A sampling-based computational strategy for the representation of epistemic uncertainty in model predictions with evidence theory, Computer Methods in Applied Mechanics and Engineering 196 (2007) 3980-98.

19. J.C. Helton, J.D. Johnson, W.L. Oberkampf, C.J. Sallaberry, Representation of Analysis Results Involving Aleatory and Epistemic Uncertainty, Sandia National Laboratories, Technical Report SAND2008-4379 (Albuquerque, New Mexico, 2008).

20. K. Sentz, S. Ferson, Combination of Evidence in Dempster-Shafer Theory, Sandia National Laboratories, Technical Report SAND 2002-0835 (Albuquerque, New Mexico, 2002).

21. G. Shafer, A Mathematical Theory of Evidence (Princeton University Press, Princeton, NJ, 1976).

22. C. Baudrit, D. Dubois, Practical Representations of Incomplete Probabilistic Knowledge, Computational Statistics \& Data Analysis 51(1) (2006) 86-108.

23. C. Baudrit, D. Dubois, D. Guyonnet, Joint Propagation and Exploitation of Probabilistic and Possibilistic Information in Risk Assessment, IEEE Transactions on Fuzzy Systems 14(5) (2006) 593-608.

24. C. Baudrit, D. Dubois, N. Perrot, Representing parametric probabilistic models tainted with imprecision, Fuzzy Sets and System 159(15) (2008) 1913-1928.

25. D. Dubois, Possibility theory and statistical reasoning, Computational Statistics \& Data Analysis 51 (2006) 47-69.

26. D. Dubois, H. Prade, Possibility Theory: An Approach to Computerized Processing of Uncertainty (Plenum Press, New York, NY, 1988).

27. S. Ferson and J.G. Hajagos, Arithmetic with uncertain numbers: rigorous and (often) best possible answers, Reliability Engineering and System Safety 85 (2004) 135-152.

28. S. Ferson, W.T. Tucker, Sensitivity in risk analyses with uncertain numbers, Setauket, New York 11733, Technical Report SAND2006-2801 (Albuquerque, New Mexico, 2006).

29. S. Ferson, V. Kreinovich, J. Hajagos, W. Oberkampf and L. Ginzburg, Experimental Uncertainty Estimation and Statistics for Data Having Interval Uncertainty, Technical Report SAND2007-0939 (Setauket, New York, 2007).

30. S. Ferson, P. Van den Brink, T.L. Estes, K. Gallagher, R. O'Connor, F. Verdonck, Bounding uncertainty analyses. In: Application of uncertainty analysis to ecological risks of pesticides, eds. Warren-Hicks, W.J., A. Hart (Pensacola and Boca Raton, FL.: SETAC and CRC Press, 2010).

31. R.E. Moore, Methods and Applications of Interval Analysis (SIAM, Philapdelphia, PA, 1979). 
32. A.C. Cullen, H.C. Frey, Probabilistic Techniques in Exposure Assessment: A Handbook for Dealing with Variability and Uncertainty in Models and Inputs (Plenum Press, New York, NY, 1999).

33. K.D. Rao, H.S. Kushwaha, A.K. Verma, A. Srividya, Quantification of epistemic and aleatory uncertainties in level-1 probabilistic safety assessment studies, Reliability Engineering \& System Safety 92(7) (2007) 947-956.

34. M.H. Kalos, P.A. Whitlock, Monte Carlo methods. Volume I: Basics (Wiley, New York, NY, 1986).

35. M. Marseguerra, E. Zio, Basics of the Monte Carlo Method with Application to System Reliability (Hagen, Germany: LiLoLe-Verlag GmbH, 2002).

36. P. Baraldi, E. Zio, A Combined Monte Carlo and Possibilistic Approach to Uncertainty Propagation in Event Tree Analysis, Risk Analysis 28(5) (2008) 1309-1326.

37. C. Baudrit, D. Guyonnet, D. Dubois, Post-processing the hybrid method for addressing uncertainty in risk assessments. Journal of the Environmental Engineering Division, ASCE 131(12) (2005) 1750-1754.

38. C. Baudrit, D. Guyonnet, D. Dubois, Joint propagation of variability and imprecision in assessing the risk of groundwater contamination. Journal of Contaminant Hydrology $\mathbf{9 3}$ (2007) 72-84.

39. C. Baudrit, I. Couso, D. Dubois, Joint propagation of probability and possibility in risk analysis: toward a formal framework, Internat. J. Approx. Reasoning 45(1) (2007) 82105.

40. J.A. Cooper, S. Ferson and L. Ginzburg, Hybrid Processing of Stochastic and Subjective Uncertainty Data, Risk Analysis 16(6) (1996) 785-791.

41. R. Flage, P. Baraldi, E. Zio, T. Aven, Possibility-probability transformation in comparing different approaches to the treatment of epistemic uncertainties in a fault tree analysis, in Reliability, Risk and Safety - Proceedings of the European Safety and Reliability (ESREL) 2010 Conference, Rhodes, Greece, 5-9 September 2010, Eds. B. Ale, I.A. Papazoglu, E. Zio (Taylor \& Francis Group, London, UK, 2010) pp. 714-721.

42. D. Guyonnet, B. Bourgine, D. Dubois, H. Fargier, B. Côme, J.P. Chilès, Hybrid approach for addressing uncertainty in risk assessments, Journal of the Environmental Engineering Division, ASCE 129 (2003) 68-78.

43. E. Kentel, M.M. Aral, Probabilistic-fuzzy health risk modeling, Stoch. Envir. Res. and Risk Ass. 18 (2004) 324-338.

44. E. Kentel, M.M. Aral, Risk tolerance measure for decision-making in fuzzy analysis: a health risk assessment perspective, Stoch. Environ Res. Ris. Assess. 21 (2007) 405-417.

45. L.A. Zadeh, Fuzzy Sets, Information and Control 8(3) (1965) 338-353.

46. E. Kentel, M.M. Aral, 2D Monte Carlo versus 2D Fuzzy Monte Carlo Health Risk Assessment, Internat. J. Stochastic Environ. Res. Risk Assess 19 (2005) 86-96.

47. B. Moller, Fuzzy randomness - a contribution to imprecise probability, ZAMM - Z. Angew. Math. Mech. 84(10-11) (2004) $754-764$.

48. B. Möller, M. Beer, Fuzzy Randomness: Uncertainty in Civil Engineering and Computational Mechanics (Springer, Berlin, 2004).

49. B. Möller, M. Beer, Engineering computation under uncertainty - Capabilities of nontraditional models, Computers and Structures 86 (2008) 1024-1041.

50. B. Moller, W. Graf, M. Beer, Safety assessment of structures in view of fuzzy randomness, Computers and Structures 81 (2003) 1567-1582.

51. B. Moller, M. Beer, W. Graf, J.U. Sickert, Time-dependent reliability of textilestrengthened RC structures under consideration of fuzzy randomness, Computers and Structures 84 (2006) 585-603. 
52. C. Baudrit, D. Dubois, Comparing Methods for Joint Objective and Subjective Uncertainty Propagation with an example in a risk assessment, In: Fourth International Symposium on Imprecise Probabilities and Their Applications (ISIPTA '05), Pittsburgh, PA, USA, 20/07/2005-23/07/2005, F. G. Cozman, R. Nau, T. Seidenfeld (Eds.).

53. C. Baudrit, D. Dubois, H. Fargier, Propagation of uncertainty involving imprecision and randomness. In: Proc. of the International Conference in Fuzzy Logic and Technology (EUSFLAT03) (Zittau, Germany, 10/09/2003-12/09/2003) pp. 653-658.

54. T. Fetz, Sets of joint probability measures generated by weighted marginal focal sets, in: Proceedings of the Second International Symposium on Imprecise Probability and Their Applications, eds. G. de Cooman, T.L. Fine and T. Seidenfeld (Shaker Publishing, Maastricht, 2001) 171-178.

55. T. Fetz, M. Oberguggenberger, Propagation of uncertainty through multivariate functions in the framework of sets of probability measures, Reliability Engineering and System Safety 85 (2004) 73-87.

56. J.C. Helton, J.D. Johnson, W.L. Oberkampf, An exploration of alternative approaches to the representation of uncertainty in model predictions, Reliability Engineering and System Safety 85 (2004) 39-72.

57. S. Moral, N. Wilson, Importance sampling Monte-Carlo algorithms for the calculation of Dempster-Shafer belief, Proceedings of the 6th International Conference on Information Processing and Management of Uncertainty in Knowledge-Based Systems (IPMU'96) (1996) Vol. III, 1337-1344.

58. W.L. Oberkampf, J. C. Helton, Investigation of evidence theory for engineering applications, AIAA Non-Deterministic Approaches Forum (Denver, Colorado, 2002) paper 2002-1569.

59. W.L. Oberkampf, J. C. Helton, K. Sentz, Mathematical Representation of Uncertainty, AIAA Non-Deterministic Approaches Forum (Seattle, Washington, 2001) paper 20011645.

60. F. Tonon, Using random set theory to propagate epistemic uncertainty through a mechanical system, Reliability Engineering and System Safety 85 (2004) 169-181.

61. F. Tonon, A. Bernardini, A. Mammino, Determination of parameters range in rock engineering by means of Random Set Theory, Reliability Engineering and System Safety 70 (2000) 241-261.

62. F. Tonon, A. Bernardini, A. Mammino, Reliability analysis of rock mass response by means of Random Set Theory, Reliability Engineering and System Safety 70 (2000) 263282.

63. S. Ferson, What Monte Carlo methods cannot do, Human and Environmental Risk Assessment 2 (1996) 990-1007.

64. S. Ferson and M. A. Burman, Correlation, dependency bounds and extinction risks, Biol. Conserv. 73 (1995) 101-105.

65. S. Ferson, L.R. Ginzburg, Different methods are needed to propagate ignorance and variability, Reliability Engineering and Systems Safety 54 (1996) 133-144.

66. S. Ferson and T.F. Long, Conservative uncertainty propagation in environmental risk assessments, in Environmental Toxicology and Risk Assessment, ed. J. S. Hughes, (Philadelphia, PA: SIAM, 1994, ASTM STP 1218) pp. 97-110.

67. R.B. Nelsen, An Introduction to Copulas, Lecture Notes in Statistics, Vol. 139 (Springer, New York, 1999).

68. H.M. Regan, S. Ferson, and D. Berleant, Equivalence of five methods for bounding uncertainty, International Journal of Approximate Reasoning 36 (2004) 1-30. 
69. R.C. Williamson and T. Downs, Probabilistic arithmetic I: Numerical methods for calculating convolutions and dependency bounds, International Journal of Approximate Reasoning 4 (1990) 89-158.

70. I. Couso, S. Moral, Independence concepts in evidence theory, International Journal of Approximate Reasoning 51 (2010) 748-758.

71. I. Couso, S. Moral, P. Walley, Examples of independence for imprecise probabilities, in: Proceedings of the First International Symposium on Imprecise Probability and Their Applications, eds. G. de Cooman, F.F. Cozman, S. Moral and P. Walley (Imprecise Probabilities Project, Universiteit Gent, 1999) pp. 121-130.

72. I. Couso, S. Moral and P. Walley, A survey of concepts of independence for imprecise probabilities, Risk Decision and Policy 5 (2000) 165-181.

73. C.P. De Campos, F.G. Cozman, Computing lower and upper expectations under epistemic independence, International Journal of Approximate Reasoning 44(3) (2007) 244-260.

74. J. Vejnarová, A Thorough Comparison of Two Conditional Independence Concepts for Belief Functions, Proceedings of Workshop on the Theory of Belief Functions, Workshop on the Theory of Belief Functions (Bres, FR, 01.04.2010-02.04.2010).

75. B.B. Yaghlane, P. Smets, K. Mellouli, Belief function independence: I. The marginal case, International Journal Approximate Reasoning 29(1) (2002) 47-70.

76. B.B. Yaghlane, P. Smets, K. Mellouli, Belief function independence: II. The conditional case, International Journal Approximate Reasoning 31(1-2) (2002) 31-75.

77. G. Coletti and B. Vantaggi, Possibility theory: Conditional independence, Fuzzy Sets and Systems 157(11) (2006) 1491-1513.

78. L.M. de Campos, J.F. Huete, Independence concepts in possibility theory: Part I, Fuzzy Sets and Systems 103(1) (1999) 127-152.

79. L.M. de Campos, J.F. Huete, Independence concepts in possibility theory: Part II, Fuzzy Sets and Systems 103(3) (1999) 487-505.

80. G. De Cooman, Possibility theory III: possibilistic independence, International Journal of General Systems 25 (1997) 353-371.

81. E. Miranda and G. De Cooman, Epistemic independence in numerical possibility theory, International Journal of Approximate Reasoning 32(1) (2003) 23-42.

82. D. Dubois, H. Prade, S. Sandri, On possibility/probability transformations, in: Fuzzy Logic: State of the Art, eds. R. Lowen, M. Roubens (Kluwer Academic Publishers, Dordrecht, 1993) 103-112.

83. P. Smets, Constructing the pignistic probability function in a context of uncertainty, in: Uncertainty in Artificial Intelligence 5, eds. M. Henrion, R.D. Shachter, L.N. Kanal, J.F. Lemmer (North-Holland, 1990) pp. 29-39.

84. D. Dubois, L. Foulloy, G. Mauris, H. Prade, Probability-possibility transformations, triangular fuzzy sets, and probabilistic inequalities, Reliable Computing 10 (2004) 273297.

85. D. Dubois, H. Prade, P. Smets, A definition of subjective possibility, International Journal of Approximate Reasoning 48 (2008) 352-364.

86. D. Dubois, H. Prade, The mean value of a fuzzy number, Fuzzy Sets and Systems 24 (1987) 279-300.

87. D. Ralescu, Average level of a fuzzy set, in: Statistical Modeling, Analysis and Management of Fuzzy Data, eds. C. Bertoluzza, M.A. Gil, D.A. Ralescu (Springer, Heidelberg, 2002) pp. 119-126.

88. M.J. Frank, R.B. Nelsen and B. Schweizer, Best-possible bounds for the distribution of a sum—a problem of Kolmogorov, Probability Theory and Related Fields 74 (1987) 199211. 
89. S. Ferson, W. Root, R. Kuhn, RAMAS Risk Calc: Risk Assessment with Uncertain Numbers, CM-113048 (EPRI, Palo Alto, CA: 1999).

\section{Appendix A. Two-dimensional Monte Carlo method}

The main steps of the procedure are: ${ }^{5,32,33}$

(1) set $i_{e}=1$ (outer loop processing epistemic uncertainty by MC simulation);

(2) sample the $i_{e}$-th set of random realizations $\boldsymbol{\theta}_{j}^{i_{i}}, j=1,2, \ldots, n$, of the epistemicallyuncertain parameter vectors $\boldsymbol{\theta}_{j}$ from the probability distributions $\boldsymbol{p}^{\boldsymbol{\theta}_{j}}\left(\boldsymbol{\theta}_{j}\right), j=1,2$, $\ldots, n$;

(3) sample $N_{a}$ random realizations $y_{j}^{i_{j} i_{e}}, i_{a}=1,2, \ldots, N_{a}, j=1,2, \ldots, n$, of the "probabilistic" variables $Y_{j}, j=1,2, \ldots, n$, from the probability distributions $p_{\theta_{j}^{\prime}}^{Y_{j}}\left(y_{j}\right), j=1,2, \ldots, n$, conditioned at the values $\boldsymbol{\theta}_{j}^{i}$ of the epistemically-uncertain parameters $\boldsymbol{\theta}_{j}$ sampled at step (2) above (inner loop processing aleatory uncertainty by MC simulation);

(4) calculate the values $z^{i_{a}, i_{e}}$ of the model output $Z$ as $z^{i_{a} i_{e}}=$ $f\left(y_{1}^{i_{a}, i_{e}}, y_{2}^{i_{a} i_{e}}, \ldots, y_{j}^{i_{o}, i_{e}}, \ldots, y_{n}^{i_{o}, i_{e}}\right), i_{a}=1,2, \ldots, N_{a}$, and build the $i_{e}$-th empirical Cumulative Distribution Function (CDF) $F_{i_{e}}^{Z}$ of $Z$;

(5) if $i_{e}<N_{e}$, set $i_{e}=i_{e}+1$ and go back to step (2) above; otherwise, go to (6) below;

(6) post-process the $N_{e}$ empirical CDFs $F_{i_{e}}^{Z}, i_{e}=1,2, \ldots, N_{e}$, thereby obtained in order to identify the upper and lower CDFs for $Z$ as $\bar{F}^{z_{i}}\left(z_{i}\right)=\max _{i_{i}=1,2, \ldots, N_{e}}\left\{F_{i_{e}}^{z_{i}}\left(z_{i}\right)\right\}$ and $\underline{F}^{z_{i}}\left(z_{i}\right)=\min _{i_{i}=1,2, \ldots, N_{e}}\left\{F_{i_{e}}^{z_{i}}\left(z_{i}\right)\right\}$, respectively (i.e., as the two "extreme" CDFs that envelop all the $N_{e}$ CDFs generated in correspondence of the $N_{e}$ realizations of epistemic uncertainty).

As highlighted in Sec. 2.1, the random samplings performed at steps (2) and (3) above may account for possible dependences existing between the epistemicallyuncertain parameters (step (2)) and between the aleatory variables (step (3)), respectively; on the other hand, such dependences can be obviously included in the analysis, only if they can be modeled within a classical MC framework. ${ }^{63}$ 
By way of example and only for illustration purposes, let us consider two random variables $Y_{1}$ and $Y_{2}$ that are described by probability distributions $p_{\theta_{1}}^{Y_{1}}\left(y_{1}\right)$ and $p_{\theta_{2}}^{Y_{2}}\left(y_{2}\right)$ (resp., CDFs $F_{\boldsymbol{\theta}_{1}}^{Y_{1}}\left(y_{1}\right)$ and $F_{\boldsymbol{\theta}_{2}}^{Y_{2}}\left(y_{2}\right)$ ) whose parameter vectors $\boldsymbol{\theta}_{1}$ and $\boldsymbol{\theta}_{2}$ are themselves affected by epistemic uncertainty. In particular, $Y_{1}$ is represented by a lognormal distribution $\operatorname{LN}\left(\boldsymbol{\theta}_{1}\right)=\operatorname{LN}\left(\theta_{1,1}, \theta_{1,2}\right)=L N\left(\mu_{1}, \sigma_{1}\right)$, where $\theta_{1,2}=\sigma_{1}=1.5$ and $\theta_{1,1}=\mu_{1}$ is described on its turn by a normal probability distribution $p^{\theta_{1,1}}\left(\theta_{1,1}\right)=p^{\mu_{1}}\left(\mu_{1}\right)$ (resp., CDF $\left.F^{\theta_{1,1}}\left(\theta_{1,1}\right)=F^{\mu_{1}}\left(\mu_{1}\right)\right)$ with mean equal to 8 , standard deviation equal to 1 and support [6, 10]; $Y_{2}$ is represented by a lognormal distribution $\operatorname{LN}\left(\boldsymbol{\theta}_{2}\right)=\operatorname{LN}\left(\theta_{2,1}, \theta_{2,2}\right)=\operatorname{LN}\left(\mu_{2}, \sigma_{2}\right)$, where $\theta_{2,2}=\sigma_{2}=1.7$ and $\theta_{2,1}=\mu_{2}$ is described on its turn by a normal probability distribution $p^{\theta_{2,1}}\left(\theta_{2,1}\right)=p^{\mu_{2}}\left(\mu_{2}\right)$ (resp., $\operatorname{CDF} F^{\theta_{2,1}}\left(\theta_{2,1}\right)=F^{\mu_{2}}\left(\mu_{2}\right)$ ) with mean equal to 2.2, standard deviation equal to 1 and support [0, 4.5]. In Figs. A.1 and A.2 the procedures for sampling the random realizations $y_{1}^{i_{a}, i_{e}}$ and $y_{2}^{i_{a}, i_{e}}$ for $Y_{1}$ and $Y_{2}$, respectively, are illustrated with respect to different assumptions of (in)dependence between the epistemically-uncertain parameters $\theta_{1,1}=\mu_{1}$ and $\theta_{2,1}=\mu_{2}$. In Fig. A.1, we assume total dependence between the epistemically-uncertain parameters $\theta_{1,1}=\mu_{1}$ and $\theta_{2,1}=\mu_{2}$ and independence between the random variables $Y_{1}$ and $Y_{2}$. With reference to the procedure outlined above, a random vector $\left\{r_{1,1}^{i_{i}}, r_{2,1}^{i_{s}}\right\}$ is sampled to process epistemic uncertainty (step (2)); in case of total dependence between the epistemically-uncertain parameters $\theta_{1,1}=\mu_{1}$ and $\theta_{2,1}=\mu_{2}$ the vector $\left\{r_{1,1}^{i_{e}}, r_{2,1}^{i_{e}}\right\}$ has to be such that $r_{1,1}^{i_{e}}=r_{2,1}^{i_{e}}$ (e.g., $r_{1,1}^{i_{e}}=r_{2,1}^{i_{e}}=0.9$ in Fig. A.1, top). The corresponding realizations $\theta_{1,1}^{i_{e}}=\mu_{1}^{i_{e}}$ and $\theta_{2,1}^{i_{e}}=\mu_{2}^{i_{e}}$ for $\theta_{1,1}=\mu_{1}$ and $\theta_{2,1}=\mu_{2}$ are then found by the inverse transform method as $\left[F^{\theta_{1,1}}\right]^{-1}\left(r_{1,1}^{i_{i}}\right)=$ $\left[F^{\mu_{1}}\right]^{-1}\left(r_{1,1}^{i_{c}}\right)(=9.18$ in Fig. A.1, top left $)$ and $\left[F^{\theta_{2,1}}\right]^{-1}\left(r_{2,1}^{i_{e}}\right)=\left[F^{\mu_{2}}\right]^{-1}\left(r_{2,1}^{i_{e}}\right)(=3.44$ in Fig. A.1, top right), respectively. The CDFs $F_{\theta_{1}}^{Y_{1}}=F_{\theta_{1,1}}^{Y_{1}}=F_{\mu_{1}}^{Y_{1}}$ and $F_{\theta_{2}}^{Y_{2}}=F_{\theta_{2,1}}^{Y_{1}}=F_{\mu_{2}}^{Y_{2}}$ for the random variables $Y_{1}$ and $Y_{2}$, respectively, are constructed using the values sampled (at step (2) above) for $\theta_{1,1}=\mu_{1}$ and $\theta_{2,1}=\mu_{2}$, i.e., $\theta_{1,1}^{i_{e}}=\mu_{1}^{i_{e}}=9.18$ and $\theta_{2,1}^{i_{e}}=\mu_{2}^{i_{e}}=3.44$ (Fig. A.1 bottom). Then, since independence is assumed between the random variables $Y_{1}$ and $Y_{2}$, two (possibly different) random numbers $u_{1}^{i_{a}}$ and $u_{2}^{i_{a}}$ (e.g., $u_{1}^{i_{a}}=0.2$ and $u_{2}^{i_{a}}=0.95$ in 
Fig. A.1 bottom) are sampled from a uniform distribution in $[0,1)$ and the corresponding realizations $y_{1}^{i_{a}, i_{e}}$ and $y_{2}^{i_{a}, i_{e}}$ of $Y_{1}$ and $Y_{2}$ are computed as $\left[F_{\theta_{1.1}^{i_{1}}}^{Y_{1}}\right]^{-1}\left(u_{1}^{i_{a}}\right)=\left[F_{\mu_{1}^{i_{e}}}^{Y_{Y_{1}}}\right]^{-1}\left(u_{1}^{i_{a}}\right)=$ $\left[F_{9.18}^{Y_{1}}\right]^{-1}(0.2)(=7.91$ in Fig. A.1, bottom left $)$ and $\left[F_{\theta_{2,1}^{i_{2}}}^{Y_{2}}\right]^{-1}\left(u_{2}^{i_{a}}\right)=\left[F_{\mu_{2}^{i_{2}}}^{Y_{2}}\right]^{-1}\left(u_{2}^{i_{a}}\right)=$ $\left[F_{3.44}^{Y_{2}}\right]^{-1}(0.95)$ (= 6.65 in Fig. A.1, bottom right), respectively.

A different situation arises in Fig. A.2, where independence is now assumed between the epistemically-uncertain parameters $\mu_{1}=\theta_{1,1}$ and $\mu_{2}=\theta_{2,1}$ (whereas independence is still assumed between the random variables $Y_{1}$ and $Y_{2}$ ). In this case, the random vector $\left\{r_{1,1}^{i_{e}}, r_{2,1}^{i_{e}}\right\}$ sampled to process epistemic uncertainty (step (2) above) is such that $r_{1,1}^{i_{e}}$ is possibly different from $r_{2,1}^{i_{e}}$ (e.g., $r_{1,1}^{i_{e}}=0.2$ and $r_{2,1}^{i_{e}}=0.75$ in Fig. A.2, top). The corresponding realizations $\theta_{1,1}^{i_{e}}=\mu_{1}^{i_{e}}$ and $\theta_{2,1}^{i_{e}}=\mu_{2}^{i_{e}}$ for $\theta_{1,1}=\mu_{1}$ and $\theta_{2,1}=\mu_{2}$ are then found as $\left[F^{\theta_{1,1}}\right]^{-1}\left(r_{1,1}^{i_{e}}\right)=\left[F^{\mu_{1}}\right]^{-1}\left(r_{1,1}^{i_{e}}\right)(=7.21$ in Fig. A.2, top left $)$ and $\left[F^{\theta_{2,1}}\right]^{-1}\left(r_{2,1}^{i_{e}}\right)=$ $\left[F^{\mu_{2}}\right]^{-1}\left(r_{2,1}^{i_{c}}\right)\left(=2.86\right.$ in Fig. A.2, top right), respectively. The CDFs $F_{\theta_{1}}^{Y_{1}}=F_{\theta_{1,1}}^{Y_{1}}=F_{\mu_{1}}^{Y_{1}}$ and $F_{\theta_{2}}^{Y_{2}}=F_{\theta_{2,1}}^{Y_{1}}=F_{\mu_{2}}^{Y_{2}}$ for $Y_{1}$ and $Y_{2}$, respectively, are constructed using the values sampled for $\theta_{1,1}=\mu_{1}$ and $\theta_{2,1}=\mu_{2}$, i.e., $\theta_{1,1}^{i_{c}}=\mu_{1}^{i_{c}}=7.21$ and $\theta_{2,1}^{i_{c}}=\mu_{2}^{i_{e}}=2.86$ (Fig. A.2 bottom). Then, since independence is still assumed between $Y_{1}$ and $Y_{2}$, as above two random numbers $u_{1}^{i_{a}}$ and $u_{2}^{i_{a}}$ (e.g., $u_{1}^{i_{a}}=0.35$ and $u_{2}^{i_{a}}=0.60$ in Fig. A.2 bottom) are sampled from a uniform distribution in $[0,1)$ and the corresponding realizations $y_{1}^{i_{a}, i_{e}}$ and $y_{2}^{i_{a}, i_{e}}$ of $Y_{1}$ and $Y_{2}$ are computed as $\left[F_{\theta_{1,1}^{i_{1}}}^{Y_{1}}\right]^{-1}\left(u_{1}^{i_{a}}\right)=\left[F_{\mu_{1}^{i}}^{Y_{1}}\right]^{-1}\left(u_{1}^{i_{a}}\right)=\left[F_{7.21}^{Y_{1}}\right]^{-1}(0.35)(=6.51$ in Fig. A.2, bottom left) and $\left[F_{\theta_{2,1}^{i}}^{Y_{2}}\right]^{-1}\left(u_{2}^{i_{a}}\right)=\left[F_{\mu_{2}^{i_{2}}}^{Y_{2}}\right]^{-1}\left(u_{2}^{i_{a}}\right)=\left[F_{2.86}^{Y_{2}}\right]^{-1}(0.60)(=2.83$ in Fig. A.2, bottom right), respectively. 

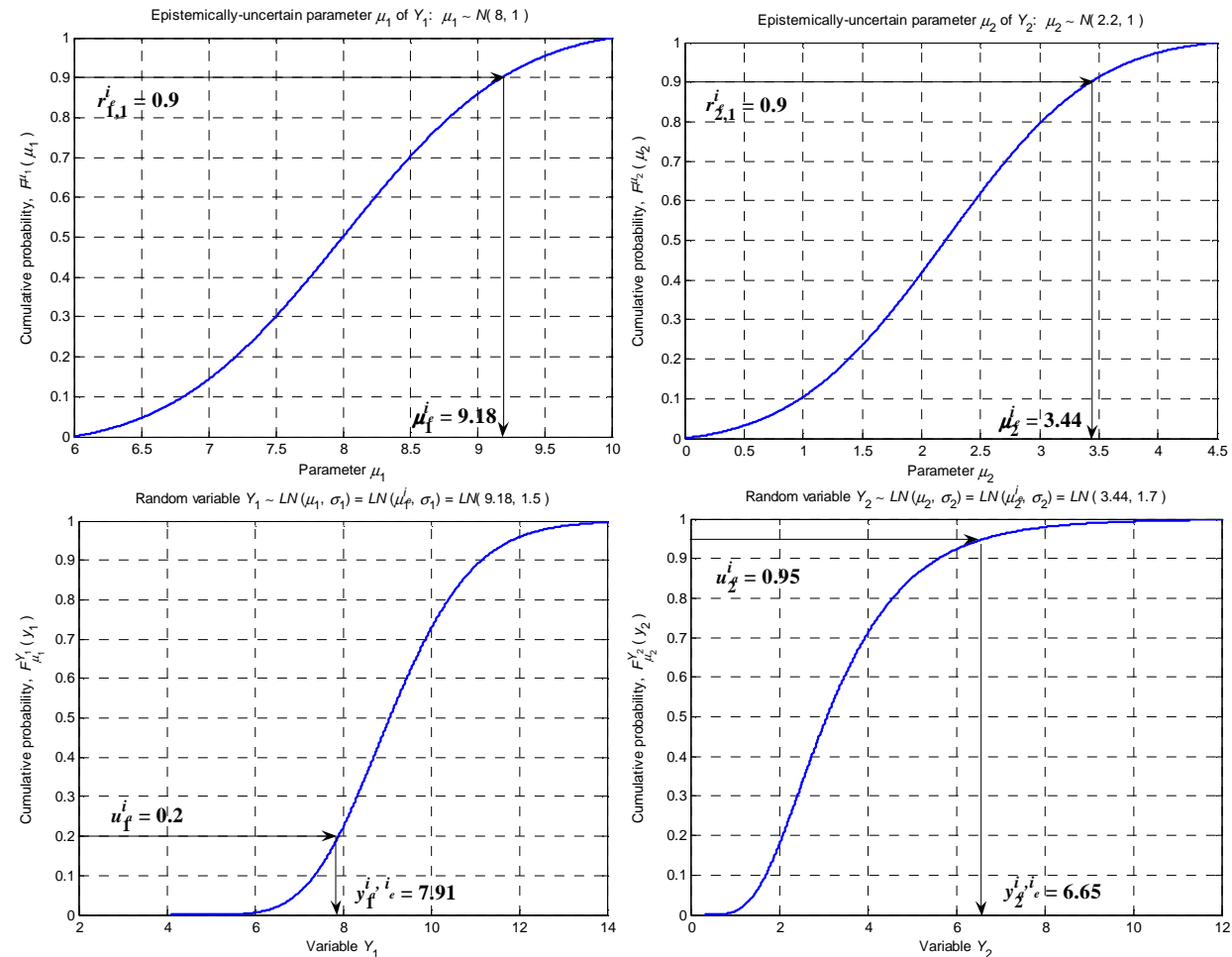

Fig. A.1. Top: PDFs $p^{\mu_{1}}\left(\mu_{1}\right)$ (left) and $p^{\mu_{2}}\left(\mu_{2}\right)$ (right) of the epistemically-uncertain parameters $\mu_{1}$ and $\mu_{2}$ of the (aleatory) PDFs of the random variables $Y_{1}$ and $Y_{2}$, respectively; in evidence, two realizations $\mu_{1}^{i}=9.18$ and $\mu_{2}=3.44$ sampled assuming total dependence between the parameters. Bottom: CDFs $F_{u}^{\gamma_{1}}$ (left) and $F_{3}^{v_{3}}$ (right) of $Y_{1}$ and $Y_{2}$ built in correspondence of $\mu_{1}=9.18$ and $\mu_{2}=3.44$, respectively; in evidence, two realizations $y_{1}^{i, i}=7.91$ and $y_{2}^{i, i}=6.65$ sampled assuming independence between $Y_{1}$ and $Y_{2}$ 

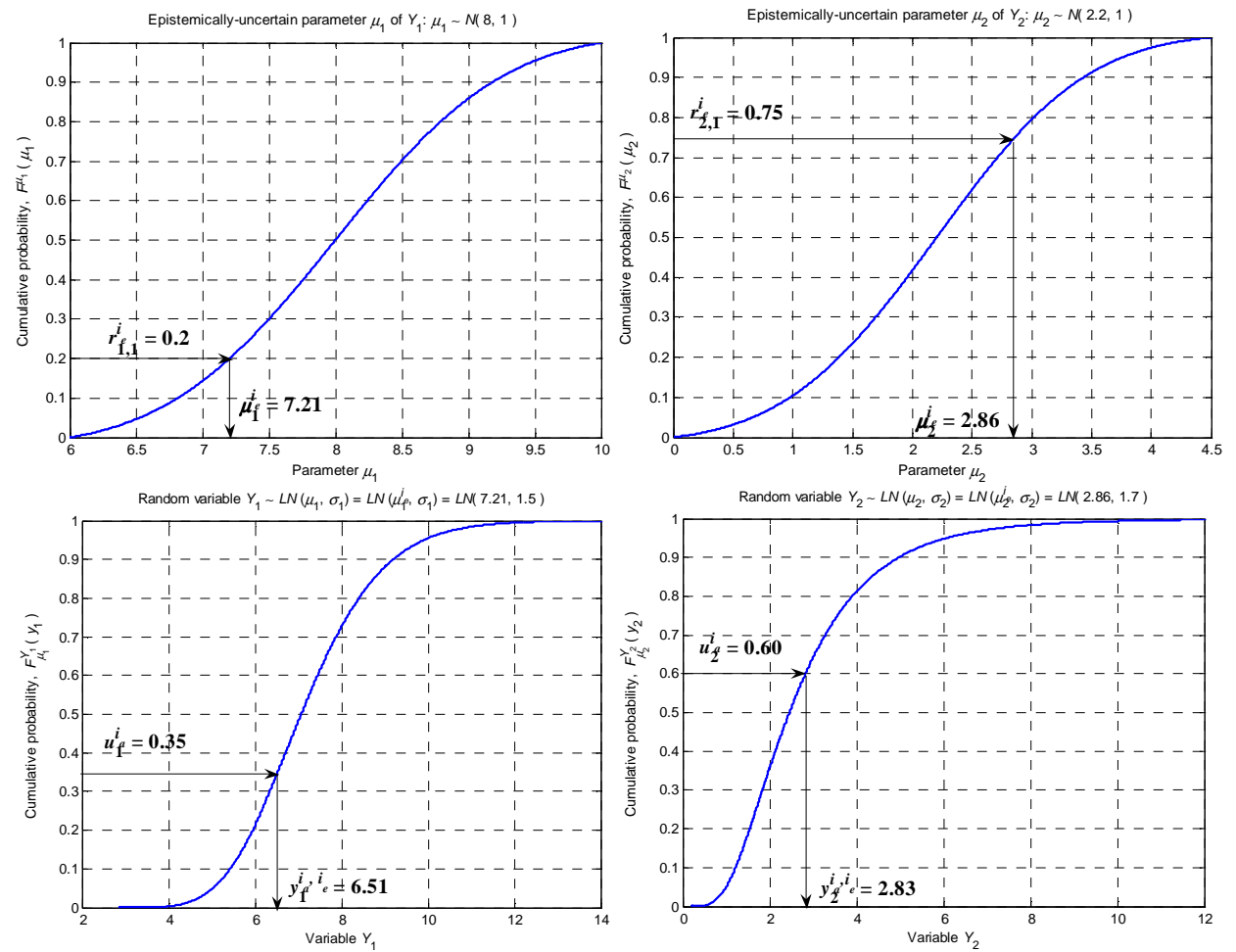

Fig. A.2. Top: PDFs $p^{\mu_{1}}\left(\mu_{1}\right)$ (left) and $p^{\mu_{2}}\left(\mu_{2}\right)$ of the epistemically-uncertain parameters $\mu_{1}$ and $\mu_{2}$ of the (aleatory) PDFs of the random variables $Y_{1}$ and $Y_{2}$, respectively; in evidence, two realizations $\mu_{1}^{i_{i}}=7.21$ and $\mu_{2}^{i_{s}}=2.86$ sampled assuming independence between the parameters. Bottom: CDFs $F^{\gamma_{1}}$ (left) and $F^{\gamma_{2}}$ (right) of $Y_{1}$ and $Y_{2}$ built in correspondence of $\mu_{1}^{i_{i}}=7.21$ and $\mu_{2}^{i_{i}}=2.86$; in evidence, two realizations $y_{1}^{i_{i} i_{e}} \stackrel{\mu_{2}}{=} 6.51$ and $y_{2}^{i_{0}, i_{i}}=2.83$ sampled assuming independence between $Y_{1}$ and $Y_{2}$

\section{Appendix B. Hybrid Monte Carlo and Fuzzy Interval Analysis approach}

The main steps of the procedure are: ${ }^{24,34-51}$

(1) set $\alpha=0$ (outer loop processing epistemic uncertainty by fuzzy interval analysis);

(2) select the $\alpha$-cuts $A_{\alpha}^{\theta_{j, 1}}, A_{\alpha}^{\theta_{j, 2}}, \ldots, A_{\alpha}^{\theta_{j, m_{j}}}, j=1,2, \ldots, n$, of the possibility distributions $\boldsymbol{\pi}^{\theta_{j}}\left(\boldsymbol{\theta}_{j}\right)=\left\{\pi^{\theta_{j, 1}}\left(\theta_{j, 1}\right), \pi^{\theta_{j, 2}}\left(\theta_{j, 2}\right), \ldots, \pi^{\theta_{j, m_{j}}}\left(\theta_{j, m_{j}}\right)\right\}$ of the parameters $\boldsymbol{\theta}_{j}, j=1,2, \ldots, n ;$

(3) sample $N_{a}$ random intervals $\left[\underline{y}_{j, \alpha}^{i_{a}}, \bar{y}_{j, \alpha}^{i_{a}}\right], i_{a}=1,2, \ldots, N_{a}, j=1,2, \ldots, n$, of the "probabilistic" variables $Y_{j}, j=1,2, \ldots, n$, letting parameters $\boldsymbol{\theta}_{j}$ range within the corresponding $\alpha$-cuts $A_{\alpha}^{\theta_{j, 1}}, A_{\alpha}^{\theta_{j, 2}}, \ldots, A_{\alpha}^{\theta_{j, m_{j}}}, j=1,2, \ldots, n$ (found at step (2) above) (inner loop processing aleatory uncertainty by standard MC simulation); 
(4) find the smallest and largest values of $Z=f\left(Y_{1}, Y_{2}, \ldots, Y_{n}\right)$, denoted by $\underline{z}_{\alpha}^{i_{\alpha}}$ and $\bar{z}_{\alpha}^{i_{\alpha}}$, respectively, letting variables $Y_{j}$ range within the intervals $\left[\underline{y}_{j, \alpha}^{i_{a}}, \bar{y}_{j, \alpha}^{i_{a}}\right]$, $i_{a}=1,2, \ldots, N_{a}, j=1,2, \ldots, n$ (found at step (3) above). Notice that if the function $Z=f\left(Y_{1}, Y_{2}, \ldots, Y_{n}\right)$ is non-monotonic, the smallest and largest values of $Z$, i.e., $\underline{z}_{\alpha}^{i_{\alpha}}$ and $\bar{z}_{\alpha}^{i_{\alpha}}$, have to be found by performing an exhaustive search within the intervals $\left[\underline{y}_{j, \alpha}^{i_{a}}, \bar{y}_{j, \alpha}^{i_{a}}\right]$ (e.g., by means of optimization algorithms, Monte Carlo Simulation, ...). Instead, when $Z=f\left(Y_{1}, Y_{2}, \ldots, Y_{n}\right)$ is monotonic (like in the case studies of Sec. 3), $\underline{z}_{\alpha}^{i_{\alpha}}$ and $\bar{z}_{\alpha}^{i_{\alpha}}$ can be found analytically in correspondence of the extreme bounds of the intervals $\left[\underline{y}_{j, \alpha}^{i_{\alpha}}, \bar{y}_{j, \alpha}^{i_{a}}\right]$ : for example, if $Z=Y_{1} \cdot Y_{2} / Y_{3}$, then $\underline{z}_{\alpha}^{i_{\alpha}}=$ $\underline{y}_{1, \alpha}^{i_{a}} \cdot \underline{y}_{2, \alpha}^{i_{\alpha}} / \bar{y}_{3, \alpha}^{i_{a}}$ and $\bar{z}_{\alpha}^{i_{\alpha}}=\bar{y}_{1, \alpha}^{i_{\alpha}} \cdot \bar{y}_{2, \alpha}^{i_{\alpha}} / \underline{y}_{3, \alpha}^{i_{a}}$

(5) take the values $\underline{z}_{\alpha}^{i_{a}}$ and $\bar{z}_{\alpha}^{i_{a}}$ found in (4) above as the lower and upper limits of the $N_{a}$ $\alpha$-cuts $A_{\alpha}^{Z, i_{a}}$ of $Z, i_{a}=1,2, \ldots, N_{a}$. A probability mass $m\left(A_{\alpha}^{Z, i_{a}}\right)=1 / N_{a}$ is associated at each $\alpha$-cut $A_{\alpha}^{Z, i_{a}}, i_{a}=1,2, \ldots, N_{a} ;$

(6) for each set $A$ of interest in the universe of discourse $U_{Z}$ of $Z$, calculate the plausibility $P l_{\alpha}(A)$ and belief $\operatorname{Bel}_{\alpha}(A)$ of level $\alpha$ as $P l_{\alpha}(A)=\sum_{A_{\alpha}^{Z, l_{\alpha}} \cap A \neq 0} m\left(A_{\alpha}^{Z, i_{\alpha}}\right)$ and $\operatorname{Bel}_{\alpha}(A)=\sum_{A_{\alpha}^{Z, t_{a}} \subseteq A} m\left(A_{\alpha}^{Z, i_{\alpha}}\right)$, respectively.

(7) if $\alpha<1$, then set $\alpha=\alpha+\Delta \alpha$ (e.g., $\Delta \alpha=0.05$ in this paper) and return to step (2) above; otherwise, go to (8) below;

(8) calculate the plausibility $P l(A)$ and belief $\operatorname{Bel}(A)$ for $A$ as $\int_{0}^{1} P l_{\alpha}(A) d \alpha \approx$ $\frac{1}{q+1} \sum_{i_{e}=0}^{q} P l_{i_{e} \cdot \Delta \alpha}(A)$ and $\int_{0}^{1} B e l_{\alpha}(A) d \alpha \approx \frac{1}{q+1} \sum_{i_{e}=0}^{q} \operatorname{Bel}_{i_{e} \cdot \Delta \alpha}(A)$, respectively, where $(q$ $+1)=(1 / \Delta \alpha+1)=21$ is the total number of $\alpha$-cuts processed in the analysis. ${ }^{24,39}$

As highlighted in Sec. 2.2, it is worth noting that performing an interval analysis on $\alpha$-cuts assumes total dependence between the epistemically-uncertain parameters. Actually, this procedure implies strong dependence between the information sources 
(e.g., the experts or observers) that supply the input possibility distributions, because the same confidence level $(1-\alpha)$ is chosen to build the $\alpha$-cuts for all the epistemicallyuncertain parameters. ${ }^{23}$ In addition, notice that the random sampling performed at step (3) above may account for possible dependences existing between the aleatory variables; on the other hand, such dependences can be obviously included in the analysis, only if they can be modeled within a classical MC framework: ${ }^{63}$ in this work, standard MC simulation is used to propagate the aleatory uncertainties, which presupposes independence between the random variables. Finally, as highlighted in Ref. 23, it is worth noting that this hybrid propagation method clearly assumes independence between the group of probabilistic (i.e., aleatory or random) variables and the group of the possibilistic (i.e., epistemically-uncertain) parameters of the aleatory probability distributions.

By way of example and only for illustration purposes, let us consider two random variables $Y_{1}$ and $Y_{2}$ that are described by probability distributions $p_{\theta_{1}}^{Y_{1}}\left(y_{1}\right)$ and $p_{\theta_{2}}^{Y_{2}}\left(y_{2}\right)$ (resp., CDFs $F_{\boldsymbol{\theta}_{1}}^{Y_{1}}\left(y_{1}\right)$ and $\left.F_{\boldsymbol{\theta}_{2}}^{Y_{2}}\left(y_{2}\right)\right)$ whose parameter vectors $\boldsymbol{\theta}_{1}$ and $\boldsymbol{\theta}_{2}$ are themselves affected by epistemic uncertainty. In particular, $Y_{1}$ is represented by a lognormal distribution $L N\left(\boldsymbol{\theta}_{1}\right)=\operatorname{LN}\left(\theta_{1,1}, \theta_{1,2}\right)=L N\left(\mu_{1}, \sigma_{1}\right)$, where $\sigma_{1}=\theta_{1,2}=1.5$ and $\theta_{1,1}=\mu_{1}$ is described by a triangular possibility distribution $\pi^{\theta_{1.1}}\left(\theta_{1,1}\right)=\pi^{\mu_{1}}\left(\mu_{1}\right)=\operatorname{TR}\left(a_{1}, c_{1}, b_{1}\right)$ with core $c_{1}=8$ and support $\left[a_{1}, b_{1}\right]=[7,10] ; Y_{2}$ is represented by a lognormal distribution $L N\left(\boldsymbol{\theta}_{2}\right)=L N\left(\theta_{2,1}, \theta_{2,2}\right)=L N\left(\mu_{2}, \sigma_{2}\right)$, where $\theta_{2,1}=\mu_{2}=9$ and $\theta_{2,2}=\sigma_{2}$ is described by a triangular possibility distribution $\pi^{\theta_{2,2}}\left(\theta_{2,2}\right)=\pi^{\sigma_{2}}\left(\sigma_{2}\right)=T R\left(a_{2}, c_{2}, b_{2}\right)$ with core $c_{2}=1.7$ and support $\left[a_{2}, b_{2}\right]=[1,2]$. In Fig. B.1 the procedure for sampling the $i_{a}$-th random intervals $\left[\underline{y}_{1, \alpha}^{i_{\alpha}}, \bar{y}_{1, \alpha}^{i_{a}}\right]$ and $\left[\underline{y}_{2, \alpha}^{i_{a}}, \bar{y}_{2, \alpha}^{i_{a}}\right]$ for the aleatory variables $Y_{1}$ and $Y_{2}$, respectively, is illustrated. A single possibility value $\alpha$ (e.g., $\alpha=0.2$ in Fig. B.1, top) is selected and the corresponding $\alpha$-cuts $A_{\alpha}^{\theta_{1,1}}=A_{\alpha}^{\mu_{1}}=A_{0.2}^{\mu_{1}}$ and $A_{\alpha}^{\theta_{2,2}}=A_{\alpha}^{\sigma_{2}}=A_{0.2}^{\sigma_{2}}$ for $\theta_{1,1}=\mu_{1}$ and $\theta_{2,2}=\sigma_{2}$ are found as $\left[\underline{\theta}_{1,1, \alpha}, \bar{\theta}_{1,1, \alpha}\right]=\left[\underline{\mu}_{1, \alpha}, \bar{\mu}_{1, \alpha}\right]=[7.2,9.6]$ and $\left[\underline{\theta}_{2,2, \alpha}, \bar{\theta}_{2,2, \alpha}\right]=\left[\underline{\sigma}_{2, \alpha}, \bar{\sigma}_{2, \alpha}\right]=[1.14$, 1.94], respectively. Then, since independence is assumed between $Y_{1}$ and $Y_{2}$, two random numbers $u_{1}^{i_{a}}$ and $u_{2}^{i_{a}}$ (e.g., $u_{1}^{i_{a}}=0.45$ and $u_{2}^{i_{a}}=0.85$ in Fig. B.1, bottom) are sampled from a uniform distribution in $[0,1)$ and the intervals $\left[\underline{y}_{1, \alpha}^{i_{a}}, \bar{y}_{1, \alpha}^{i_{a}}\right]$ and $\left[\underline{y}_{2, \alpha}^{i_{a}}, \bar{y}_{2, \alpha}^{i_{a}}\right]$ are 


$$
\begin{aligned}
& \text { computed } \\
& {\left[\inf _{\theta_{1} \in\left[\underline{\theta}_{1, \alpha}, \overline{\boldsymbol{\theta}}_{1, \alpha]}\right]}\left[F_{\theta_{1}}^{Y_{1}}\right]^{-1}\left(u_{1}^{i_{a}}\right), \sup _{\boldsymbol{\theta}_{1} \in\left[\underline{\theta}_{1, \alpha}, \bar{\theta}_{1, \alpha}\right]}\left[F_{\theta_{1}}^{Y_{1}}\right]^{-1}\left(u_{1}^{i_{a}}\right)\right]} \\
& {\left[\inf _{\mu_{1} \in\left[\underline{\mu}_{1, \alpha}, \bar{\mu}_{1, \alpha}\right]}\left[F_{\mu_{1}}^{Y_{1}}\right]^{-1}\left(u_{1}^{i_{\alpha}}\right), \sup _{\mu_{1} \in \underline{\left.\mu_{1, \alpha}, \bar{\mu}_{1, \alpha}\right]}}\left[F_{\mu_{1}}^{Y_{1}}\right]^{-1}\left(u_{1}^{i_{\alpha}}\right)\right]=\left[\inf _{\mu_{k} \in[7.2,9.6]}\left[F_{\mu_{1}}^{Y_{1}}\right]^{-1}(0.45), \sup _{\mu_{1} \in[7.2,9.6]}\left[F_{\mu_{1}}^{Y_{1}}\right]^{-1}(0.45)\right]=}
\end{aligned}
$$

$[10.19,11]$, respectively.
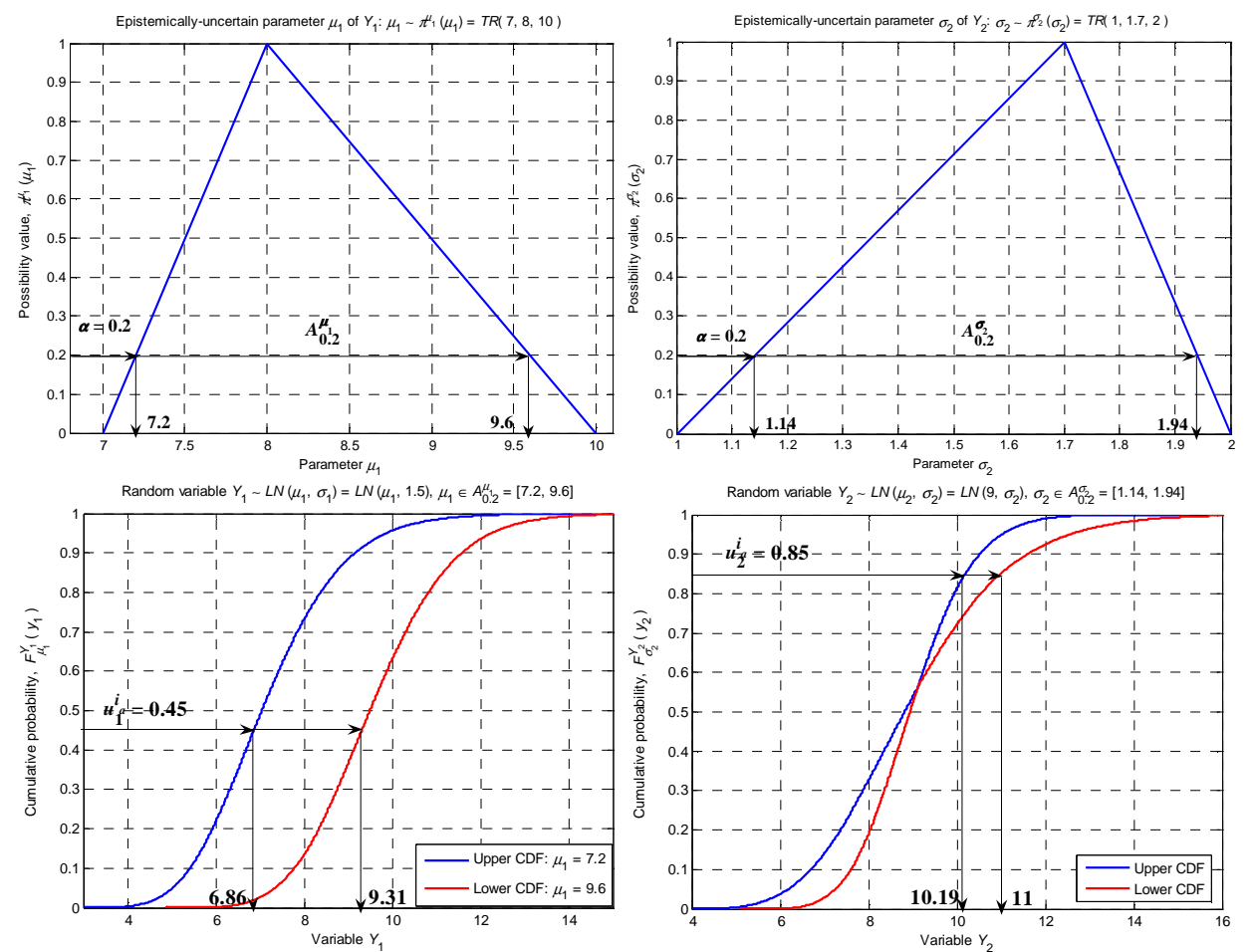

Fig. B.1. Top: possibility distributions $\pi^{\mu_{1}}\left(\mu_{1}\right)$ (left) and $\pi^{\sigma_{2}}\left(\sigma_{2}\right)$ (right) of the epistemically-uncertain parameters $\mu_{1}$ and $\sigma_{2}$ of the (aleatory) PDFs of the random variables $Y_{1}$ and $Y_{2}$, respectively; in evidence the $\alpha$ cuts of level $\alpha=0.2 A_{\alpha}^{\mu_{\mu}}=\left[\mu, \bar{\mu}_{1, \alpha}\right]=[7.2,9.6]$ and $A_{\alpha}^{\sigma_{2}}=\left[\sigma_{2 \alpha}, \bar{\sigma}_{2 \alpha}\right]=[1.14,1.94]$, respectively. Bottom: upper and lower CDFs of $Y_{1}\left(\bar{l}\right.$ (eft) and $Y_{2}$ (right) built in correspondence of the extreme values $\mu=7.2$ and $\bar{\mu}_{1, \alpha}=9.6$ of the $\alpha$-cut $A_{\alpha}^{\mu_{1}}$ and the extreme values $\underline{\sigma}_{2, \alpha}=1.14$ and $\bar{\sigma}_{2, \alpha}=1.94$ of the $\alpha$-cut $A_{\alpha}^{\sigma_{2}}$, respectively; in

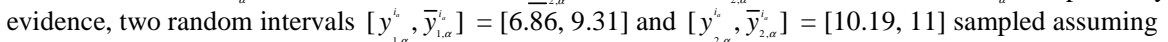
independence between $Y_{1}$ and $Y_{2}$ 


\section{Appendix C. Monte Carlo-based Dempster-Shafer approach employing Independent Random Sets}

In the MC-based DS-IRS approach, the possibility distribution of a generic epistemicallyuncertain parameter $\theta$ is encoded into discrete (focal) sets $A_{\alpha_{t}}=\left[\underline{\theta}_{\alpha_{t}}, \bar{\theta}_{\alpha_{t}}\right], t=1,2, \ldots, q$ (where $\alpha_{1}=1>\alpha_{2}>\ldots>\alpha_{q}>\alpha_{q+1}=0$ ) whose masses are $m_{\alpha_{t}}=\Delta \alpha_{t}=\alpha_{t}-\alpha_{t+1}$ (see Sec. 2.3). In this paper, $q=20$ and $m_{\alpha_{t}}=\Delta \alpha_{t}=\alpha_{t}-\alpha_{t+1}=0.05$ for the sake of comparison with the hybrid MC-FIA approach of Sec. 2.2 and Appendix B.

The main steps of the procedure are: ${ }^{52,53}$

(1) set $i_{e}=1$ (outer loop processing epistemic uncertainty by standard MC simulation);

(2) sample the values $\left\{\alpha_{j, i}^{i_{e}}\right\}, i=1,2, \ldots, m_{j}, j=1,2, \ldots, n$, from the discrete distribution $\left\{\left(\alpha_{j, i, t}, m_{\alpha_{j, i, t}}\right): t=1,2, \ldots, q\right\}:$ these sampled values represent the $\alpha$ levels of the focal sets $A_{\alpha_{j, i}^{\prime, j}}^{\theta_{j, 1}}, A_{\alpha_{j, i}^{\prime, 2}}^{\theta_{j, 2}}, \ldots, A_{\alpha_{f, i}^{\prime}}^{\theta_{j, m_{j}}}, \quad i=1,2, \ldots, m_{j}, \quad j=1,2, \ldots, n$, of the discretized possibility distributions $\boldsymbol{\pi}^{\boldsymbol{\theta}_{j}}\left(\boldsymbol{\theta}_{j}\right)$ of the parameters $\boldsymbol{\theta}_{j}, j=1,2, \ldots, n$;

(3) perform the same steps (3) - (4) (inner loop processing aleatory uncertainty by standard MC simulation) as in the procedure of Appendix B to obtain $\underline{z}^{i_{e}, i_{a}}$ and $\bar{z}^{i_{e}, i_{a}}, i_{a}=1,2, \ldots, N_{a}$, as the upper and lower limits of $Z=f\left(Y_{1}, Y_{2}, \ldots, Y_{n}\right)$ in correspondence of the $i_{e}$-th random realization of epistemic uncertainty;

(4) if $i_{e}<N_{e}$, set $i_{e}=i_{e}+1$ and go back to step (2) above; otherwise, go to (5) below;

(5) the random sets $E^{i_{e}, i_{a}}=\left[\underline{z}^{i_{e}, i_{a}}, \bar{z}^{i_{e}, i_{a}}\right]$ of $Z$ are obtained with the collection of the values $\underline{z}^{i_{e}, i_{a}}$ and $\bar{z}^{i_{e}, i_{a}}, i_{e}=1,2, \ldots, N_{e}, i_{a}=1,2, \ldots, N_{a}$, found at step (3) above. A probability mass $m\left(E^{i_{e}, i_{a}}\right)=1 /\left(N^{a} \cdot N^{e}\right)$ is associated at each random set $E^{i_{e}, i_{a}}, i_{e}=$ $1,2, \ldots, N_{e}, i_{a}=1,2, \ldots, N_{a} ;$

(6) calculate the plausibility $\operatorname{Pl}(A)$ and belief $\operatorname{Bel}(A)$ for each set $A$ of interest contained in the universe of discourse $U_{Z}$ of $Z$ as $P l(A)=\sum_{E^{i_{c}, l_{a}} \cap A \neq 0} m\left(E^{i_{c}, i_{a}}\right)$ and $\operatorname{Bel}(A)=\sum_{E^{t_{e}, i_{a}} \subseteq A} m\left(E^{i_{e}, i_{a}}\right)$, respectively.

Notice that, differently from the hybrid MC-FIA approach, at step (2) above a different possibility value (resp., confidence level) $\alpha$ (resp., $1-\alpha$ ) is randomly and independently sampled for each epistemically-uncertain parameter, i.e., random set independence is assumed between the epistemically-uncertain parameters. In addition, 
notice that the random sampling performed at step (3) above may account for possible dependences existing between the aleatory variables; on the other hand, such dependences can be obviously included in the analysis, only if they can be modeled within a classical MC framework: ${ }^{63}$ in this work, standard MC simulation is used to propagate the aleatory uncertainties, which presupposes independence between the random variables.

By way of example and only for illustration purposes, let us consider the two random variables $Y_{1}$ and $Y_{2}$ described in the previous Appendix B. In Fig. C.1 the procedure for sampling the $i_{a}$-th random intervals $\left[\underline{y}_{1}^{i_{a}}, \bar{y}_{1}^{i_{a}}\right]$ and $\left[\underline{y}_{2}^{i_{a}}, \bar{y}_{2}^{i_{a}}\right]$ for the aleatory variables $Y_{1}$ and $Y_{2}$, respectively, is illustrated. Since independence is now assumed between the epistemically-uncertain parameters $\theta_{1,1}=\mu_{1}$ and $\theta_{2,2}=\sigma_{2}$, two possibly different possibility values $\alpha_{1,1}^{i}$ and $\alpha_{2,2}^{i}$ (e.g., $\alpha_{1,1}^{i}=0.8$ and $\alpha_{2,2}^{i}=0.1$ in Fig. C.1, top) are randomly selected and the corresponding focal sets $A_{\alpha_{j_{11}}}^{\theta_{1,1}}=A_{\alpha_{1,}^{\prime}}^{\mu_{1}}=A_{0.8}^{\mu_{1}}$ and $A_{\alpha_{2_{2}}}^{\theta_{2,2}}=A_{\alpha_{\delta_{2}}}^{\sigma_{2}}=$

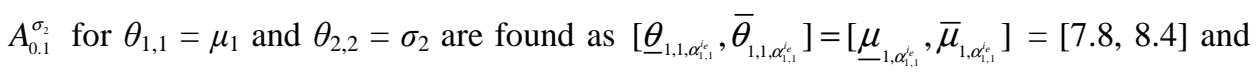
$\left[\underline{\theta}_{2,2, \alpha_{2,2}^{j_{2}}}, \bar{\theta}_{2,2, \alpha_{\alpha_{2}}}\right]=\left[\underline{\sigma}_{2, \alpha_{2,2}^{j_{2}}}, \bar{\sigma}_{2, \alpha_{2,2}^{\prime}}\right]=[1.07,1.97]$, respectively. Then, since independence is also assumed between $Y_{1}$ and $Y_{2}$, two random numbers $u_{1}^{i_{i}}$ and $u_{2}^{i_{s}}$ (e.g., $u_{1}^{i_{i}}=0.55$ and $u_{2}^{i_{a}}=0.15$ in Fig. C.1, bottom) are sampled from a uniform distribution in $[0,1)$ and the

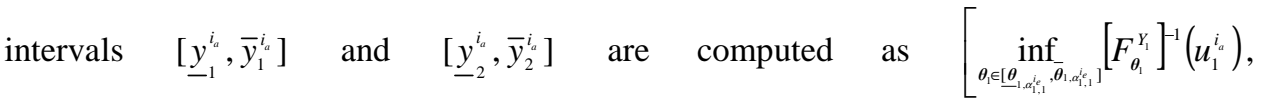

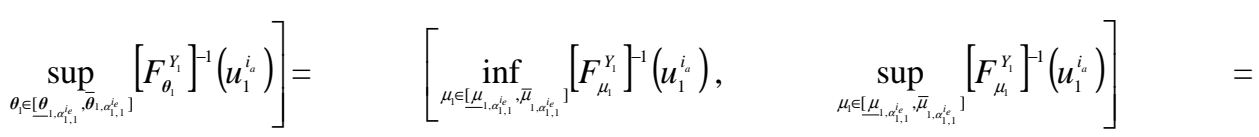

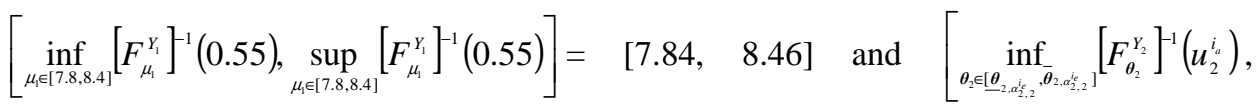

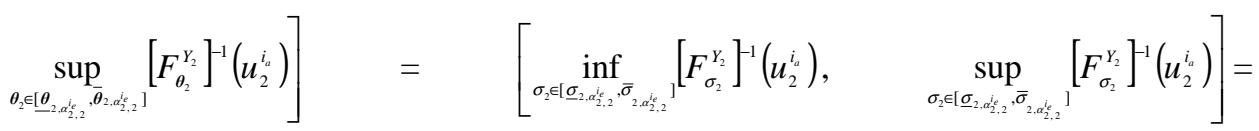
$\left[\inf _{\sigma_{2}[1.107,1.97]}\left[F_{\sigma_{2}}^{Y_{2}}\right]^{-1}(0.15), \sup _{\sigma_{\varepsilon}[1.07,1.97]}\left[F_{\sigma_{2}}^{Y_{2}}\right]^{-1}(0.15)\right]=[7.02,7.90]$, respectively. 

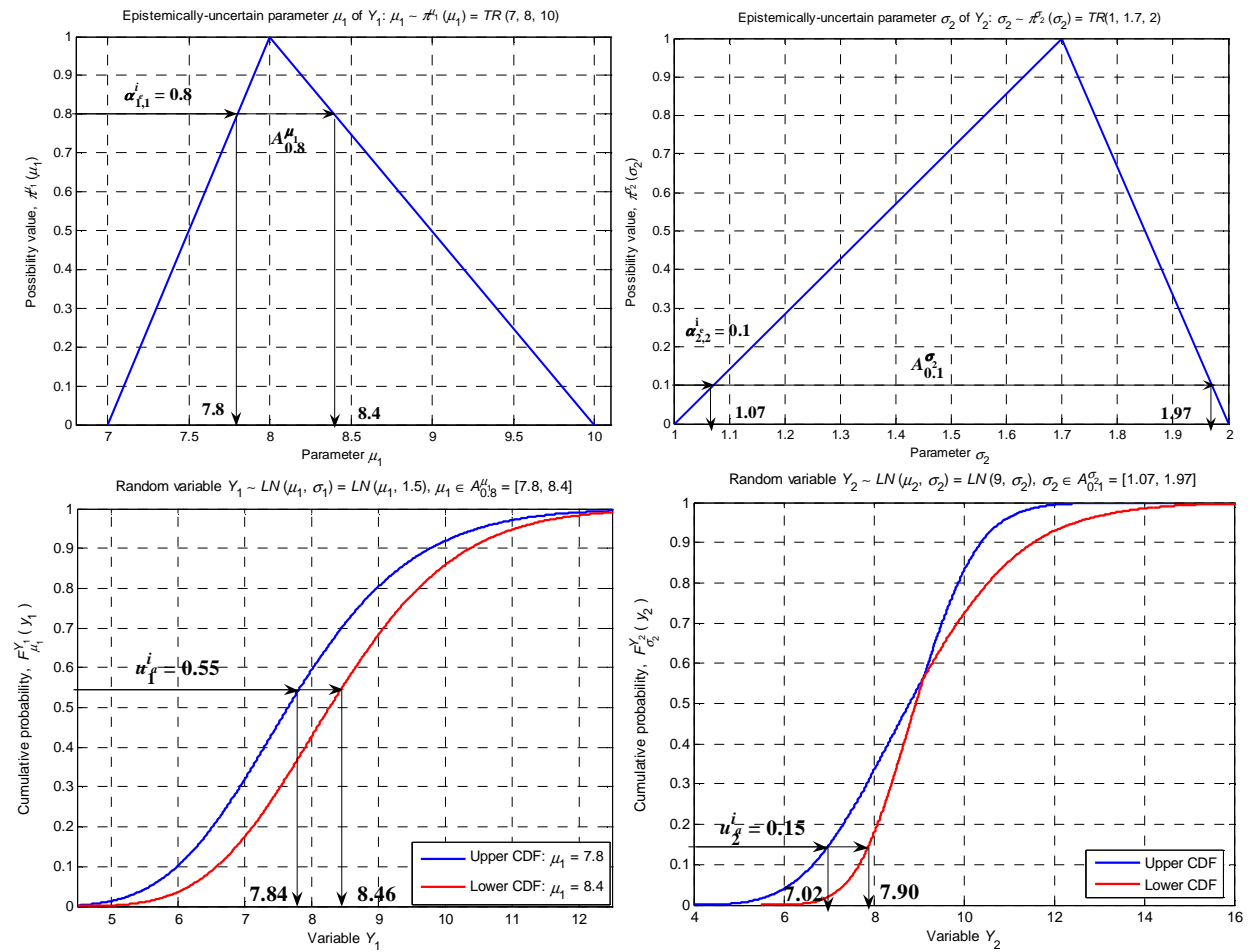

Fig. C.1. Top: possibility distributions $\pi^{\mu_{1}}\left(\mu_{1}\right)$ (left) and $\pi^{\sigma_{2}}\left(\sigma_{2}\right)$ (right) of the epistemically-uncertain parameters $\mu_{1}$ and $\sigma_{2}$ of the (aleatory) PDFs of the random variables $Y_{1}$ and $Y_{2}$, respectively; in evidence the $\alpha$ -

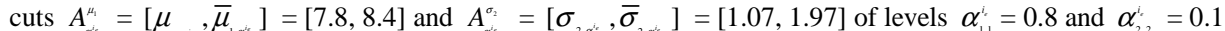
for $\mu_{1}$ and $\sigma_{2}$, respectively. Bottom: upper and lower CDFs of $Y_{1}$ (left) and $Y_{2}$ (right) built in correspondence of the extreme values $\mu=7.8$ and $\bar{\mu}_{1, \alpha}=8.4$ of the $\alpha$-cut $A_{\alpha}^{\mu}$ and the extreme values $\sigma_{2, \alpha}=1.07$ and $\bar{\sigma}_{2, \alpha}=$

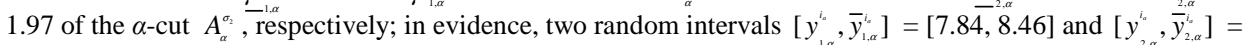
$[7.02,7.90]$ sampled assuming independence between $\bar{Y}_{1}$ and $Y_{2}$, respectively

\section{Appendix D. Hybrid Dependency Bound Convolution and Fuzzy Interval Analysis approach}

The Dependency Bound Convolution (DBC) method ${ }^{69}$ allows computing extreme upper and lower CDFs on the outputs of probabilistic models no matter what correlations or dependencies exist among the input variables; these bounds are also the "pointwise best possible." 17,68

The method is based on the theorem of Frank et al., 1987, ${ }^{88}$ which provides the pointwise best possible bounds $\bar{F}_{D B C}^{Z}$ and $\underline{F}_{D B C}^{Z}$ for the result $Z=O\left(Y_{1}, Y_{2}\right)$ of a generic binary mathematical operation $O\left(Y_{1}, Y_{2}\right)$ (which is non-decreasing in each place and continuous, except possibly at infinity) between almost surely positive random variables $Y_{1}$ and $Y_{2}$ of given Cumulative Distribution Functions $(\mathrm{CDFs}) F^{Y_{1}}$ and $F^{Y_{2}} \cdot{ }^{17,68}$ The 
reader is referred to Refs. 17, 68 and 69 for the formal expressions of $\bar{F}_{D B C}^{Z}$ and $\underline{F}_{D B C}^{Z}$ when $O \in\left\{+,-,{ }^{*}, /\right\}$ and to Ref. 89 for the extension of these results to other operators such as power, logarithm, and so on.

In this theoretical framework, Ref. 69 provides operative rules to explicitly determine the bounds $\bar{F}_{D B C}^{Z}$ and $\underline{F}_{D B C}^{Z}$ when $O \in\{+,-, *, /\}$. In particular, the main steps of the algorithm are: (i) discretize the CDFs $F^{Y_{1}}$ and $F^{Y_{2}}$ of the random variables $Y_{1}$ and $Y_{2}$ into upper and lower CDFs $\bar{F}^{Y_{1}}, \bar{F}^{Y_{2}}, \underline{F}^{Y_{1}}$, and $\underline{F}^{Y_{2}}$, respectively (i.e., build probability boxes for $Y_{1}$ and $Y_{2}$ ); (ii) apply the mathematical formulas provided in Ref. 69 to obtain bounds $\left[\bar{F}_{D B C}^{Z}\right]^{-1}$ and $\left[F_{D B C}^{Z}\right]^{-1}$ on the quantile function $\left[F^{Z}\right]^{-1}$; (iii) take the inverse of $\left[\bar{F}_{D B C}^{Z}\right]^{-1}$ and $\left[\underline{F}_{D B C}^{Z}\right]^{-1}$ to get the bounds $\bar{F}_{D B C}^{Z}$ and $\underline{F}_{D B C}^{Z}$ on the CDF $F^{z}$. The reader is referred to the seminal paper for further technical details.

The characteristics of the algorithm described above are here exploited to join the Dependency Bound Convolution (DBC) method and the Fuzzy Interval Analysis (FIA) approach within a "level-2" framework of hybrid (probabilistic and possibilistic) uncertainty propagation. In synthesis, the main steps of the hybrid DBC-FIA algorithm are:

(1) outer loop processing epistemic uncertainty by FIA: perform the same steps (1) and (2) of the procedure in Appendix B to get the $\alpha$-cuts $A_{\alpha}^{\theta_{j, 1}}, A_{\alpha}^{\theta_{j, 2}}, \ldots, A_{\alpha}^{\theta_{j, m_{j}}}$ of the possibility distributions $\boldsymbol{\pi}^{\boldsymbol{\theta}_{j}}\left(\boldsymbol{\theta}_{j}\right)=\left\{\boldsymbol{\pi}^{\theta_{j, 1}}\left(\theta_{j, 1}\right), \pi^{\theta_{j, 2}}\left(\theta_{j, 2}\right), \ldots, \pi^{\theta_{j, m_{j}}}\left(\theta_{j, m_{j}}\right)\right\}$ of the parameters $\boldsymbol{\theta}_{j}, j=1,2, \ldots, n$;

(2) inner loop processing aleatory uncertainty by DBC:

(i) letting parameters $\boldsymbol{\theta}_{j}$ range within the corresponding $\alpha$-cuts $A_{\alpha}^{\theta_{j, 1}}, A_{\alpha}^{\theta_{j, 2}}, \ldots, A_{\alpha}^{\theta_{j, m, j}}$ (found at step (1) above), build the upper and lower CDFs $\bar{F}_{\alpha}^{Y_{j}}, \underline{F}_{\alpha}^{Y_{j}}$ (i.e., the probability boxes) of level $\alpha$ for the "probabilistic" variables $Y_{j}$ as $\bar{F}_{\alpha}^{Y_{j}}=$ $\sup _{\boldsymbol{\theta}_{i} \in A_{\alpha}^{\theta_{j}}}\left\{F_{\boldsymbol{\theta}_{j}}^{Y_{j}}\right\}$ and $\underline{F}_{\alpha}^{Y_{j}}=\inf _{\boldsymbol{\theta}_{j} \in A_{\alpha}^{\theta_{j}}}\left\{F_{\boldsymbol{\theta}_{j}}^{Y_{j}}\right\}, j=1,2, \ldots, n$, where $F_{\boldsymbol{\theta}_{j}}^{Y_{j}}$ is the CDF of $p_{\boldsymbol{\theta}_{j}}^{Y_{j}}$; 
(ii) apply the DBC rules to obtain the bounds of level $\alpha\left[\bar{F}_{\alpha, D B C}^{Z}\right]^{-1}$ and $\left[F_{\alpha, D B C}^{Z}\right]^{-1}$ on the quantile function $\left[F^{Z}\right]^{-1}$ of the model output $Z=f\left(Y_{1}, Y_{2}, \ldots, Y_{n}\right)$;

(iii) take the inverse of $\left[\bar{F}_{\alpha, D B C}^{z}\right]^{-1}$ and $\left[\underline{F}_{\alpha, D B C}^{Z}\right]^{-1}$ to get the bounds of level $\alpha \bar{F}_{\alpha, D B C}^{z}$ and $\underline{F}_{\alpha, D B C}^{Z}$ on the CDF $F^{Z}$ of the model output $Z=f\left(Y_{1}, Y_{2}, \ldots, Y_{n}\right)$.

(3) repeat step (2) above for another possibility value $\alpha \in(0,1]$.

The bounds $\bar{F}_{D B C}^{Z}$ and $\underline{F}_{D B C}^{Z}$ on $F^{Z}$ can be computed as $\bar{F}_{D B C}^{Z}=\int_{0}^{1} \bar{F}_{\alpha, D B C}^{z} d \alpha$ and $\underline{F}_{D B C}^{Z}=\int_{0}^{1} \underline{F}_{\alpha, D B C}^{Z} d \alpha$, respectively. 